\title{
Half-Isomorfismos de Loops Automórficos
}

\author{
Giliard Souza dos Anjos \\ TESE APRESENTADA \\ $\mathrm{AO}$ \\ Instituto DE Matemática E EstatísticA \\ DA \\ UNIVERSIDADE DE \\ SÃO PAULO \\ PARA \\ OBTENÇÃO DO TÍTULO \\ $\mathrm{DE}$ \\ DOUTOR EM CIÊNCIAS
}

\author{
Programa: Doutorado em Matemática \\ Orientador: Prof ${ }^{\mathrm{a}}$. Dr ${ }^{\mathrm{a}}$. Maria de Lourdes Merlini Giuliani
}

Durante o desenvolvimento deste trabalho o autor recebeu auxílio financeiro da CAPES

São Paulo, Maio de 2018 


\section{Half-Isomorfismos de Loops Automórficos}

Esta versão da tese contém as correções e alterações sugeridas pela Comissão Julgadora durante a defesa da versão original do trabalho, realizada em 09/03/2018. Uma cópia da versão original está disponível no Instituto de Matemática e Estatística da Universidade de São Paulo.

Comissão Julgadora:

- $\operatorname{Prof}^{\mathrm{a}}$. Dr ${ }^{\mathrm{a}}$. Maria de Lourdes Merlini Giuliani (orientadora) - UFABC

- Prof. Dr. Alexandre Grichkov - IME-USP

- Prof. Dr. Henrique Guzzo Junior - IME-USP

- Prof. Dr. Plamen Emilov Kochloukov - UNICAMP

- Prof ${ }^{\mathrm{a}}$. Dr ${ }^{\mathrm{a}}$. Dylene Agda Souza de Barros - UFU 


\section{Resumo}

ANJOS, G. S. Half-isomorfismos de Loops Automórficos. 2018. Tese - Instituto de Matemática e Estatística, Universidade de São Paulo, São Paulo, 2018.

Loops automórficos, ou $A$-loops, são loops nos quais todas as aplicações internas são automorfismos. Esta variedade de loops inclui grupos e loops de Moufang comutativos. Loops automórficos diedrais formam uma classe de $A$-loops construída a partir da duplicação de grupos abelianos finitos, generalizando a construção do grupo diedral. Outra classe de A-loops é a dos loops automórficos de Lie, construída a partir de anéis de Lie, definindo-se uma nova operação entre seus elementos. Um half-isomorfismo é uma bijeção $f$ entre loops $L$ e $L^{\prime}$ onde, para quaisquer $x$ e $y$ pertencentes a $L$, temos que $f(x y)$ pertence ao conjunto $\{f(x) f(y), f(y) f(x)\}$. Dizemos que o half-isomorfismo $f$ é não trivial quando $f$ não é um isomorfismo e nem um anti-isomorfismo. Nesta tese descrevemos propriedades de half-isomorfismos de loops, classificamos os half-isomorfismos entre loops automórficos diedrais e obtivemos o grupo de half-automorfismos nesta classe. Para os loops automórficos de Lie de ordem ímpar, mostramos que todo half-automorfismo é trivial.

Palavras-chave: Loop, loop automórfico, half-isomorfismo, half-automorfismo, grupo de halfautomorfismos, loop automórfico diedral, loop automórfico de Lie, anel de Lie. 


\section{Abstract}

ANJOS, G. S. Half-isomorphisms of Automorphic Loops. 2018. Tese - Instituto de Matemática e Estatística, Universidade de São Paulo, São Paulo, 2018.

Automorphic loops, or $A$-loops, are loops in which every inner mapping is an automorphism. This variety of loops includes groups and commutative Moufang loops. Dihedral automorphic loops form a class of $A$-loops, constructed from the duplication of finite abelian groups, that generalizes the construction of the dihedral group. Another class of $A$-loops is the Lie automorphic loops, constructed from Lie rings, where a new operation between its elements is defined. A half-isomorphism is a bijection $f$ between loops $L$ and $L^{\prime}$ where, for any $x$ and $y$ belong to $L$, we have that $f(x y)$ belongs to the set $\{f(x) f y), f(y) f(x)\}$. We say that half-isomorphism $f$ is non trivial when $f$ is neither an isomorphism nor an anti-isomorphism. In this thesis, we describe properties of halfisomorphisms of loops, we classify the half-isomorphisms between dihedral automorphic loops and we obtain the group of half-automorphisms in this class. For the Lie automorphic loops of odd order, we show that every half-automorphism is trivial.

Keywords: Loop, automorphic loop, half-isomorphism, half-automorphism, group of halfautomorphisms, dihedral automorphic loop, Lie automorphic loop, Lie ring. 


\section{Sumário}

Lista de Símbolos $\quad$ vii

1 Introdução $\quad 1$

2 Conceitos básicos $\quad 3$

2.1 Loop . . . . . . . . . . . . . . . . . . . . . . . . . . 3

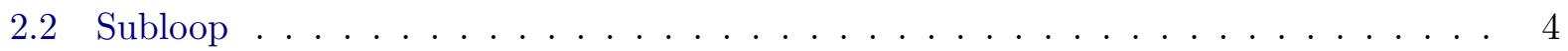

2.3 Loop potencialmente associativo . . . . . . . . . . . . . . . . . . . 9

2.4 Loops de Moufang . . . . . . . . . . . . . . . . . . . . 11

2.5 Loops automórficos . . . . . . . . . . . . . . . . . . . . . . . . 12

3 Half-isomorfismos de loops $\quad 15$

3.1 Definição e exemplo . . . . . . . . . . . . . . . . . . . . . . 15

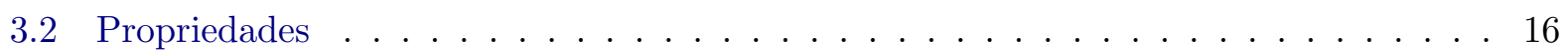

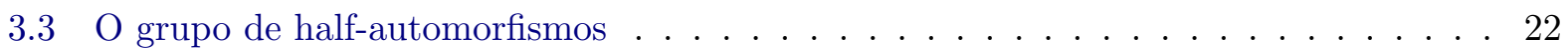

4 Half-isomorfismos de loops automórficos diedrais $\quad 23$

4.1 Loop automórfico diedral . . . . . . . . . . . . . . . . . . . . . 23

4.2 Half-isomorfismos entre $L_{\alpha}$ e $L_{\beta} \quad \ldots \ldots \ldots \ldots \ldots \ldots \ldots \ldots$

4.3 O grupo de half-automorfismos de $L_{\alpha} \ldots \ldots \ldots \ldots \ldots$

5 Half-automorfismos de loops automórficos de Lie $\quad 43$

5.1 Loop automórfico de Lie . . . . . . . . . . . . . . . . . . . . . . . 43

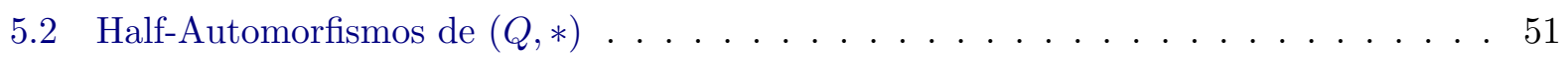

$\begin{array}{ll}\text { Referências Bibliográficas } & 63\end{array}$ 


\title{
Lista de Símbolos
}

\author{
Aut $(L) \quad$ Grupo de automorfismos de $L$ \\ $\operatorname{Mlt}(L) \quad$ Grupo das multiplicações de $L$ \\ Inn $(L) \quad$ Grupo das aplicações internas de $L$ \\ $N_{\lambda}(L) \quad$ Núcleo esquerdo de $L$ \\ $N_{\mu}(L) \quad$ Núcleo central de $L$ \\ $N_{\rho}(L) \quad$ Núcleo direito de $L$ \\ $N(L) \quad$ Núcleo de $L$ \\ $C(L) \quad$ Comutante de $L$ \\ $\mathcal{Z}(L) \quad$ Centro de $L$ \\ $\operatorname{Half}(L) \quad$ Conjunto dos half-automorfismos de $L$ \\ $\operatorname{Half}_{T}(L) \quad$ Conjunto dos half-automorfismos triviais de $L$ \\ $\operatorname{Half}_{N}(L) \quad$ Conjunto dos half-automorfismos não triviais de $L$ \\ $\operatorname{Half}\left(L, L^{\prime}\right) \quad$ Conjunto dos half-isomorfismos entre $L$ e $L^{\prime}$ \\ $\operatorname{Half}_{T}\left(L, L^{\prime}\right) \quad$ Conjunto dos half-isomorfismos triviais entre $L$ e $L^{\prime}$ \\ $\operatorname{Half}_{N}\left(L, L^{\prime}\right) \quad$ Conjunto dos half-isomorfismos não triviais entre $L$ e $L^{\prime}$ \\ $C_{L}(x) \quad$ Centralizador do elemento $x$ de um loop $L$ \\ $C_{L}(S) \quad$ Centralizador do subconjunto $S$ de um loop $L$ \\ $C l(x) \quad$ Classe de conjugação do elemento $x$ de um grupo $G$ \\ $\operatorname{Hol}(G) \quad$ Holomorfo de um grupo $G$ \\ $x^{\lambda} \quad$ inverso à esqueda do elemento $x$ de um loop \\ $x^{\rho} \quad$ inverso à direita do elemento $x$ de um loop \\ Fix $(\Omega) \quad$ Conjunto dos elementos do loop fixados pelos automorfismos do conjunto $\Omega$ \\ $G L\left(m, \mathbb{Z}_{n}\right) \quad$ Grupo linear geral das matrizes quadradas de tamanho $m$ com entradas em $\mathbb{Z}_{n}$ \\ $S L\left(m, \mathbb{Z}_{n}\right) \quad$ Anel de Lie das matrizes quadradas de tamanho $m$ com entradas em $\mathbb{Z}_{n}$ de \\ traço zero
}




\section{Capítulo 1}

\section{Introdução}

Um conjunto não vazio $L$ com uma operação binária $*$ é chamado um loop se em $L$ existe um elemento identidade 1 tal que $1 * x=x=x * 1$ para todo $x$ pertecente a $L$; e para todo $a$ e $b$ pertecentes a $L$, existem únicos $x$ e $y$ pertecentes a $L$ tais que $a * x=b$ e $y * a=b$. Grupos são loops associativos. Alguns resultados não são verificados para loops em geral, como por exemplo, os teoremas de Lagrange, teorema de Cauchy e teoremas de Sylow, enquanto que o teorema do homomorfismo é válido para a categoria de loops. Loops de Moufang são loops onde vale a identidade de Moufang $x *((y * z) * x)=(x * y) *(z * x)$. Tais loops foram introduzidos por R. Moufang em 1934 [Mou35] e é a classe de loops não associativos mais estudada. Os loops de Moufang são diassociativos (isto é, quaisquer dois elementos geram um subloop associativo), sendo essa sua propriedade mais relevante.

Para $a$ pertecente a $L$, definimos a translação à direita por a como sendo a bijeção $R_{a}: L \rightarrow L$; $x \rightarrow x * a$; e, de forma análoga, definimos a translação à esquerda por a como sendo a bijeção $L_{a}: L \rightarrow L ; x \rightarrow a * x$. Estas translações geram o grupo das multiplicações $M l t(L)=\left\langle R_{a}, L_{a} ; a \in L\right\rangle$. O subgrupo $\operatorname{Inn}(L)=\{f \in M l t(L) ; f(1)=1\}$ de $M l t(L)$ é conhecido como grupo das aplicações internas. Um loop automórfico, ou um $A$-loop, é um loop onde toda aplicação interna é um automorfismo, isto é, $\operatorname{Inn}(L)$ é subgrupo de $\operatorname{Aut}(L)$. Loops automórficos foram definidos por R.H. Bruck e L.J. Paige [BP56] que os denominaram $A$-loops (Aqui usaremos $A$-loops para simplificar). Bruck e Paige mostraram que todo $A$-loop é potencialmente associativo, ou seja, cada elemento gera um grupo cíclico. Neste mesmo artigo eles conjecturaram que "todo $A$-loop diassociativo é um loop de Moufang", fato que só foi provado em 2002 por M.K. Kinyon, K. Kunen e J.D. Phillips [KKP02] usando ferramentas computacionais. Mais recentemente, a estrutura dos $A$-loops mereceu a investigação de vários matemáticos, destacando-se o artigo de M.K. Kinyon, K. Kunen, J.D. Phillips e P. Vojtěchovský [KKPV16]. Neste artigo foram construídas duas classes de $A$-loops. A primeira foi construída a partir da duplicação de grupos abelianos, onde obtém-se um tipo de $A$-loop que generaliza a construção do grupo diedral. Chamamos os loops desta classe de loops automórficos diedrais. A segunda classe de $A$-loops foi construída a partir de anéis de Lie seguindo a construção de C.R.B. Wright [Wri69]. Chamamos os loops desta classe de loops automórficos de Lie.

Um half-isomorfismo é uma bijeção $f$ entre loops $(L, *)$ e $\left(L^{\prime}, \cdot\right)$ onde, para quaisquer $x$ e $y$ pertencentes a $L$, temos que $f(x * y)$ pertence ao conjunto $\{f(x) \cdot f(y), f(y) \cdot f(x)\}$, e se $L=L^{\prime}$ o mesmo é chamado de half-automorfismo. No caso em que um half-isomorfismo é ou um isomorfismo ou um anti-isomorfismo, dizemos que ele é trivial. Half-isomorfismos começaram a ser estudados por W.R. Scott [Sco57] sobre grupos, onde foi mostrado que todo half-isomorfismo entre grupos é trivial. O mesmo resultado foi obtido para Loops de Moufang de ordem ímpar por S. Gagola e M.L. Merlini Giuliani [IG12], e para loops de Moufang automórficos por A. Grishkov, M.L. Merlini Giuliani, M. Rasskazova e L. Sabinina [GGRS16] no caso de half-automorfismos, sendo estes resultados generalizados por M.K. Kinyon, I. Stuhl e P. Vojtěchovský [KSV16]. Em [IG13] os autores S. Gagola e M.L. Merlini Giuliani descreveram condições para a existência de half-automorfismos não triviais entre certos tipos de loops de Moufang de ordem par. 
O objetivo deste trabalho é estender o estudo da existência de half-isomorfismos não triviais à classe dos loops automórficos, sendo o foco aqui os loops automórficos diedrais e de Lie.

No capítulo dois definimos os conceitos básicos que serão utilizados nos outros capítulos. Definimos loop potencialmente associativo, loops de Moufang, loops automórficos, e enunciamos alguns resultados sobre estes. Acrescentamos aqui a demonstração de alguns resultados que, embora consolidados, não é frequente encontrar as suas provas, como por exemplo o teorema do homomorfismo.

No capítulo três introduzimos o conceito de half-isomorfismo entre loops, ressaltando as propriedades dos loops do conjunto domínio e do conjunto contra-domínio que são preservadas por half-isomorfismos. Definimos o conceito de half-isomorfismo especial e estabelecemos condições necessárias e suficientes para que um half-isomorfismo seja deste tipo. Construímos contra-exemplos para mostrar que nem sempre a recíproca dos resultados obtidos é válida. Por fim introduzimos o conceito de grupo de half-automorfismos de um loop.

No capítulo quatro mostramos que é possivel existir half-isomorfismos não triviais entre dois loops automórficos diedrais, identificando uma condição necessária e suficiente para isso. Além disso, caracterizamos tanto os half-isomorfismos triviais quanto os não triviais entre esses $A$-loops, obtendo suas quantidades. Por fim caracterizamos o grupo de half-automorfismos nesta classe de $A$-loops.

No capítulo cinco estudamos half-automorfismos para a classe de loops automórficos de Lie. Para o caso onde essa classe de $A$-loops é de ordem ímpar e não-associativa obtemos vários resultados, destacando-se que os half-automorfismos fixam o núcleo central e preservam a propriedade flexível. O resultado principal do capítulo é o de que, em loops automórficos de Lie de ordem ímpar, todo half-automorfismo é trivial. 


\section{Capítulo 2}

\section{Conceitos básicos}

Neste capítulo introduzimos os conceitos básicos de loops e suas propriedades que serão abordados nos capítulos seguintes. As seções de 1 a 3 destinam-se às definições principais de loops e suas estruturas algébricas fundamentais. Na quarta seção introduzimos os loops de Moufang, uma classe de loops que é uma generalização de grupos e que surgem quando a propriedade associativa é substituida pela identidade de Moufang. Por fim, na quinta seção introduzimos a classe dos loops automórficos e enunciamos resultados importantes sobre esta classe de loops.

\section{$2.1 \quad$ Loop}

Definição 2.1.1. Um loop é um par ordenado $(L, *)$, onde $L$ é um conjunto não vazio e * é uma operação binária que satisfaz:

(i) Existe 1 pertencente a $L$ tal que $1 * a=a * 1=a$ para todo $a$ pertencente a $L$.

(ii) Fixados elementos $a$ e $b$ pertencentes a $L$, existem únicos $x$ e $y$ pertencentes a $L$ tais que $a * x=b$ e $y * a=b$.

No caso em que $(L, *)$ também satisfaz a propriedade associativa, temos que $(L, *)$ é um grupo.

Geralmente deixaremos de indicar a operação do loop, caso ela não seja relevante, escrevendo apenas $L$ para denotar o loop $(L, *)$.

Definimos a ordem do loop $L$ como sendo a quantidade de elementos que o conjunto $L$ possui, e a denotamos por $|L|$.

Exemplo 2.1.2. Seja $L=\{1,2,3,4,5\}$ e defina em $L$ a operação $*$ dada pela seguinte tabela de multiplicação:

\begin{tabular}{|c|c|c|c|c|c|}
\hline$*$ & 1 & 2 & 3 & 4 & 5 \\
\hline 1 & 1 & 2 & 3 & 4 & 5 \\
\hline 2 & 2 & 1 & 4 & 5 & 3 \\
\hline 3 & 3 & 5 & 2 & 1 & 4 \\
\hline 4 & 4 & 3 & 5 & 2 & 1 \\
\hline 5 & 5 & 4 & 1 & 3 & 2 \\
\hline
\end{tabular}

É uma tarefa simples verificar que a tabela acima representa um loop de ordem 5 . Como $2 *(3 * 5)=2 * 4=5$ e $(2 * 3) * 5=4 * 5=1$, temos que $L$ é não associativo. Como $2 * 3=4 \mathrm{e}$ $3 * 2=5$, temos que $L$ é não comutativo.

Agora seja $L$ um loop e $x$ um elemento de $L$. Podemos observar que, da definição de loop, segue que existem únicos elementos $y$ e $z$ pertencentes a $L$ tais que $y * x=1$ e $x * z=1$. Tais elementos são chamados respectivamente de inverso à esquerda e inverso à direita de $x$, e são denotados 
respectivamente por $x^{\lambda}$ e $x^{\rho}$. No caso em que $x^{\lambda}=x^{\rho}$, denotamos este elemento por $x^{-1}$, que será chamado o inverso bilateral de $x$.

No exemplo 2.1.2, vemos que o elemento 2 de $L$ tem inverso bilateral $2^{-1}=2$, e o elemento 3 tem inversos $3^{\lambda}=5$ e $3^{\rho}=4$.

Observação 2.1.3. Segue da definição de loop que vale a lei do cancelamento, ou seja, para elementos $x, y$ e $z$ pertencentes a um loop $L$, se $x * y=x * z$ ou se $y * x=z * x$, então $y=z$.

Agora seja $L$ loop e $x$ pertencente a $L$. Defina as funções $L_{x}, R_{x}: L \longrightarrow L$ por:

$$
L_{x}(y)=x * y \quad R_{x}(y)=y * x \quad(y \in L)
$$

As funções $L_{x}$ e $R_{x}$ são chamadas respectivamente de translações à esquerda e à direita de $x$ em $L$, e são bijeções. Com isso, definimos o grupo multiplicativo de $L$, denotado por $\operatorname{Mlt}(L)$, como sendo o grupo gerado pelas bijeções $L_{x}$ e $R_{x}$, e o grupo das aplicações internas de $L$, denotado por $\operatorname{Inn}(L)$, como sendo o subgrupo de $M l t(L)$ formado pelos elementos deste que fixam a identidade. Para estes dois grupos, temos as seguintes expressões:

$\operatorname{Mlt}(L)=\left\langle R_{x}, L_{x} \mid x \in L\right\rangle$

$\operatorname{Inn}(L)=\{\varphi \in \operatorname{Mlt}(L) \mid \varphi(1)=1\}$

A partir das translações dos elementos de $L$, podemos definir também as seguintes bijeções:

$$
\begin{array}{r}
L_{(x, y)}=L_{(y x)}^{-1} \circ L_{y} \circ L_{x} \\
R_{(x, y)}=R_{(x y)}^{-1} \circ R_{y} \circ R_{x} \\
\\
T_{x}=L_{x}^{-1} \circ R_{x}
\end{array}
$$

Com elas podemos obter uma outra expressão para o grupo das aplicações internas, como é mostrado no resultado a seguir.

Proposição 2.1.4. ([Pfl90] Teo. I.5.3) Seja L um loop. Então:

$$
\operatorname{Inn}(L)=\left\langle L_{(x, y)}, R_{(x, y)}, T_{x} \mid x, y \in L\right\rangle
$$

O conceito de grupo das aplicações internas será importante para a definição de loop automórfico na seção 2.5 .

\subsection{Subloop}

Definição 2.2.1. Um subconjunto não vazio $H$ de um loop $(L, *)$ é dito ser subloop de $L$ se $(H, *)$ é loop.

No exemplo 2.1.2, $H=\{1,2\}$ é subloop de $L$. Podemos observar aqui que o teorema de Lagrange, um resultado clássico na teoria de grupos, não é verificado para este loop $L$, pois $|L|=5$ e $|H|=2$.

O resultado a seguir nos dá uma condição necessária e suficiente para um subconjunto de um loop ser um subloop. 
Proposição 2.2.2. ([GJM96] Prop. 1.2) Seja $H$ um subconjunto não vazio de um loop L. Então $H$ é subloop se, e somente se, as três condições abaixo são verificadas.

a) Se $x$ e y pertencem a $H$, então $x * y$ também pertence a $H$.

b) Se $x$ e z pertencem a $H$ e $x * y=z$, onde y pertence a $L$, então y pertence a $H$.

c) Se y e z pertencem a $H$ e $x * y=z$, onde $x$ pertence a $L$, então $x$ pertence $a H$.

No caso de loops finitos, apenas a condição a) é necessária, como podemos ver no resultado abaixo.

Proposição 2.2.3. Seja $H$ um subconjunto não vazio de um loop finito $(L, *)$. Então $H$ é subloop se, e somente se, $H$ é fechado para a operação $*$.

Demonstração. É fácil ver que se $H$ é subloop de $L$, então $H$ é fechado para *. Agora suponha que $H$ é fechado para $*$, ou seja, se $x, y \in H$, então $x * y \in H$.

Fixado $x \in H$, considere as translações $L_{x}$ e $R_{x}$. Como $H$ é fechado, segue que $L_{x}(H) \subseteq H$ e $R_{x}(H) \subseteq H$. Como $L_{x}$ e $R_{x}$ são bijeções e $L$ é finito, segue que $L_{x}(H)=R_{x}(H)=H$. Logo $L_{x}^{-1}(H)=R_{x}^{-1}(H)=H$.

Agora, sejam $x, z \in H$ e $a, b \in L$ tais que $a * x=z$ e $x * b=z$. Assim $a=R_{x}^{-1}(z)$ e $b=L_{x}^{-1}(z)$, e então $a, b \in H$. Portanto, da proposição 2.2.2 segue que $H$ é subloop de $L$.

A seguir serão definidos subloops importantes de um loop $L$.

i) Os núcleos esquerdo, central e direito de $L$, denotados respectivamente por $N_{\lambda}(L), N_{\mu}(L)$ e $N_{\rho}(L)$, são definidos por:

$N_{\lambda}(L)=\{a \in L \mid a *(x * y)=(a * x) * y \quad \forall x, y \in L\}$
$N_{\mu}(L)=\{a \in L \mid x *(a * y)=(x * a) * y \quad \forall x, y \in L\}$
$N_{\rho}(L)=\{a \in L \mid x *(y * a)=(x * y) * a \quad \forall x, y \in L\}$

ii) O núcleo e o centro de $L$, denotados respectivamente por $N(L)$ e $\mathcal{Z}(L)$, são definidos por:

$N(L)=N_{\lambda}(L) \cap N_{\mu}(L) \cap N_{\rho}(L)$

$\mathcal{Z}(L)=\{x \in N(L) \mid x * y=y * x \quad \forall y \in L\}$

Proposição 2.2.4. ([GJM96] Prop. 1.5) Seja L um loop. Então $N_{\lambda}(L), N_{\mu}(L), N_{\rho}(L)$ e $N(L)$ são subloops associativos de $L$ e $\mathcal{Z}(L)$ é um subloop associativo e comutativo de $L$.

Definição 2.2.5. Um subloop $H$ de $L$ é dito ser um subloop normal de $L$ se satisfaz, para todo $x$ e $y$ pertencentes a $L$ :

(i) $H x=x H$

(ii) $H(x * y)=(H x) y$

(iii) $(x H) y=x(H y)$

(iv) $(x * y) H=x(y H)$

Se $H$ é subloop normal de $L$, denotamos $H \triangleleft L$. 
Observação 2.2.6. Na definição acima, verifica-se facilmente que se $H$ satisfazer a condição (i), e quaisquer duas de (ii), (iii) e (iv), satisfará também a terceira.

Dado um subloop normal $H$ de um loop $L$, definimos o conjunto $L / H$ por:

$$
L / H=\{H x \mid x \in L\}
$$

e definimos a operação · neste conjunto por:

$$
H x \cdot H y=H(x * y)
$$

Analogamente com a teoria de grupos, a condição $H \triangleleft L$ garante que $L / H$ e $\cdot$ estão bem definidos e que formam um loop, chamado loop quociente de $H$ em L.

O centro $\mathcal{Z}(L)$ é um exemplo de subloop normal de um loop $L$. Assim $L / Z(L)$ é um loop quociente.

Definição 2.2.7. Sejam $(L, *)$ e $\left(L^{\prime}, \cdot\right)$ loops e $\varphi: L \rightarrow L^{\prime}$ uma função. Dizemos que:

a) $\varphi$ é um homomorfismo se $\varphi(x * y)=\varphi(x) \cdot \varphi(y)$, para todo $x$ e $y$ pertencentes a $L$.

b) $\varphi$ é um anti-homomorfismo se $\varphi(x * y)=\varphi(y) \cdot \varphi(x)$, para todo $x$ e $y$ pertencentes a $L$.

c) $\varphi$ é um isomorfismo se $\varphi$ é um homomorfismo e é bijetora.

d) $\varphi$ é um anti-isomorfismo se $\varphi$ é um anti-homomorfismo e é bijetora.

No caso em que $\varphi$ é um homomorfismo, definimos o núcleo e a imagem de $\varphi$, denotados respectivamente por $\operatorname{Ker}(\varphi)$ e $\operatorname{Im}(\varphi)$, como segue:

$\operatorname{Ker}(\varphi)=\left\{x \in L \mid \varphi(x)=1_{L^{\prime}}\right\}$

$\operatorname{Im}(\varphi)=\{\varphi(x) \mid x \in L\}$

onde $1_{L^{\prime}}$ é o elemento identidade do loop $L^{\prime}$.

Com estas definições, temos o teorema seguinte.

Teorema 2.2.8. (Teorema do Homomorfismo) Sejam $L$ e $L^{\prime}$ loops e $\varphi: L \rightarrow L^{\prime}$ um homomorfismo. Então:

$$
L / \operatorname{Ker}(\varphi) \cong \operatorname{Im}(\varphi)
$$

Demonstração. Vamos provar os itens de (i) a (iv) a seguir.

(i) $\operatorname{Ker}(\varphi)$ é subloop de $L$

Sejam $x, y \in \operatorname{Ker}(\varphi)$. Como $\varphi(x)=\varphi(y)=1_{L^{\prime}}$, temos:

$$
\varphi(x * y)=\varphi(x) \cdot \varphi(y)=1_{L^{\prime}}
$$

$\operatorname{Logo}, x * y \in \operatorname{Ker}(\varphi)$.

Agora, $\operatorname{sejam} x, z \in \operatorname{Ker}(\varphi)$ e $a, b \in L$ tais que $z=a * x=x * b$. Como $\varphi(x)=\varphi(z)=1_{L^{\prime}}$, temos: $1_{L^{\prime}}=\varphi(z)=\varphi(a * x)=\varphi(a) \cdot \varphi(x)=\varphi(a)$ 
$1_{L^{\prime}}=\varphi(z)=\varphi(x * b)=\varphi(x) \cdot \varphi(b)=\varphi(b)$

Desta forma, $a, b \in \operatorname{Ker}(\varphi)$. Logo, da proposição 2.2.2, temos que $\operatorname{Ker}(\varphi)$ é subloop de $L$.

(ii) $\operatorname{Ker}(\varphi)$ é normal em $L$

Fixados $x, y \in L$, mostraremos que as três identidades a seguir são válidas.

(ii.1) $\operatorname{Ker}(\varphi) x=x \operatorname{Ker}(\varphi)$

Fixado $h \in \operatorname{Ker}(\varphi)$, existe $w \in L$ tal que $h * x=x * w$. Assim:

$$
\varphi(x)=\varphi(h) \cdot \varphi(x)=\varphi(h * x)=\varphi(x * w)=\varphi(x) \cdot \varphi(w)
$$

Com isto, $\varphi(w)=1_{L^{\prime}}$, e temos que $\operatorname{Ker}(\varphi) x \subseteq x \operatorname{Ker}(\varphi)$.

Agora para $h \in \operatorname{Ker}(\varphi)$ fixo, existe $z \in L$ tal que $x * h=z * x$. Assim:

$$
\varphi(x)=\varphi(x) \cdot \varphi(h)=\varphi(x * h)=\varphi(z * x)=\varphi(z) \cdot \varphi(x)
$$

Desta forma, $\varphi(z)=1_{L^{\prime}}$, e temos que $x \operatorname{Ker}(\varphi) \subseteq \operatorname{Ker}(\varphi) x$. Portanto $\operatorname{Ker}(\varphi) x=x \operatorname{Ker}(\varphi)$.

(ii.2) $x(\operatorname{Ker}(\varphi) y)=(x \operatorname{Ker}(\varphi)) y$

Fixado $h \in \operatorname{Ker}(\varphi)$, existe $w \in L$ tal que $x *(h * y)=(x * w) * y$. Assim:

$$
\varphi(x) \cdot \varphi(y)=\varphi(x) \cdot(\varphi(h) \cdot \varphi(y))=\varphi(x *(h * y))=\varphi((x * w) * y)=(\varphi(x) \cdot \varphi(w)) \cdot \varphi(y)
$$

Pela lei do cancelamento, segue que $\varphi(x)=\varphi(x) \cdot \varphi(w)$, e usando novamente a lei do cancelamento, temos $\varphi(w)=1_{L^{\prime}}, \log x(\operatorname{Ker}(\varphi) y) \subseteq(x \operatorname{Ker}(\varphi)) y$.

Agora, fixado $h \in \operatorname{Ker}(\varphi)$, existe $z \in L$ tal que $(x * h) * y=x *(z * y)$. Assim:

$$
\varphi(x) \cdot \varphi(y)=(\varphi(x) \cdot \varphi(h)) \cdot \varphi(y)=\varphi((x * h) * y)=\varphi(x *(z * y))=\varphi(x) \cdot(\varphi(z) \cdot \varphi(y))
$$

Pela lei do cancelamento, segue que $\varphi(y)=\varphi(z) \cdot \varphi(y)$, e usando novamente a lei do cancelamento, temos $\varphi(z)=1_{L^{\prime}}, \operatorname{logo}(x \operatorname{Ker}(\varphi)) y \subseteq x(\operatorname{Ker}(\varphi) y)$. Portanto $x(\operatorname{Ker}(\varphi) y)=(x \operatorname{Ker}(\varphi)) y$.

(ii.3) $(\operatorname{Ker}(\varphi) x) y=\operatorname{Ker}(\varphi)(x y)$

Fixado $h \in \operatorname{Ker}(\varphi)$, existe $w \in L$ tal que $(h * x) * y=w *(x * y)$. Assim:

$$
\varphi(x) \cdot \varphi(y)=(\varphi(h) \cdot \varphi(x)) \cdot \varphi(y)=\varphi((h * x) * y)=\varphi(w *(x * y))=\varphi(w) \cdot(\varphi(x) \cdot \varphi(y))
$$

Pela lei do cancelamento, segue que $\varphi(w)=1_{L^{\prime}}$, logo $(\operatorname{Ker}(\varphi) x) y \subseteq \operatorname{Ker}(\varphi)(x y)$.

Agora, fixado $h \in \operatorname{Ker}(\varphi)$, existe $z \in L$ tal que $h *(x * y)=(z * x) * y$. Assim:

$$
\varphi(x) \cdot \varphi(y)=\varphi(h) \cdot(\varphi(x) \cdot \varphi(y))=\varphi(h *(x * y))=\varphi((z * x) * y)=(\varphi(z) \cdot \varphi(x)) \cdot \varphi(y)
$$

Pela lei do cancelamento, segue que $\varphi(x)=\varphi(z) \cdot \varphi(x)$, e usando novamente a lei do cancelamento, temos $\varphi(z)=1_{L^{\prime}}, \operatorname{logo} \operatorname{Ker}(\varphi)(x y) \subseteq(\operatorname{Ker}(\varphi) x) y$. Portanto $(\operatorname{Ker}(\varphi) x) y=\operatorname{Ker}(\varphi)(x y)$.

Assim, de (ii.1), (ii.2) e (ii.3), segue que $\operatorname{Ker}(\varphi)$ é normal em $L$.

(iii) $\operatorname{Im}(\varphi)$ é subloop de $L^{\prime}$ 
Fixados $\varphi(x), \varphi(y) \in \operatorname{Im}(\varphi)$, temos:

$$
\varphi(x) \cdot \varphi(y)=\varphi(x * y) \in \operatorname{Im}(\varphi)
$$

Agora, sejam $\varphi(x), \varphi(z) \in \operatorname{Im}(\varphi)$ e $a, b \in L^{\prime}$ tais que $\varphi(z)=a * \varphi(x)=\varphi(x) * b$.

Como $x, z \in L$, existem $c, d \in L$ tais que $z=c * x=x * d$. Logo:

$$
\varphi(z)=\varphi(c * x)=\varphi(c) \cdot \varphi(x) \quad \text { e } \quad \varphi(z)=\varphi(x * d)=\varphi(x) \cdot \varphi(d)
$$

Pela lei do cancelamento, segue que $a=\varphi(c)$ e $b=\varphi(d)$, $\operatorname{logo} a, b \in \operatorname{Im}(\varphi)$. Portanto, da proposição 2.2 .2 , temos que $\operatorname{Im}(\varphi)$ é subloop de $L^{\prime}$.

(iv) $L / \operatorname{Ker}(\varphi) \cong \operatorname{Im}(\varphi)$

Defina $\psi: L / \operatorname{Ker}(\varphi) \rightarrow \operatorname{Im}(\varphi)$ por $\psi(\operatorname{Ker}(\varphi) x)=\varphi(x)$. Vamos mostrar que $\psi$ é isomorfismo e faremos isso em quatro etapas:

(iv.1) $\psi$ está bem definida

Sejam $x, y \in L$ tais que $\operatorname{Ker}(\varphi) x=\operatorname{Ker}(\varphi) y$. Assim, $y=h * x$, onde $h \in \operatorname{Ker}(\varphi)$. Logo:

$$
\varphi(y)=\varphi(h * x)=\varphi(h) \cdot \varphi(x)=\varphi(x)
$$

Com isto, $\psi(\operatorname{Ker}(\varphi) x)=\psi(\operatorname{Ker}(\varphi) y)$, e asim $\psi$ está bem definida.

(iv.2) $\psi$ é sobrejetora

Fixado $\varphi(x) \in \operatorname{Im}(\varphi)$, temos que $\psi(\operatorname{Ker}(\varphi) x)=\varphi(x)$. Logo $\psi$ é sobrejetora.

(iv.3) $\psi$ é injetora

Sejam $\operatorname{Ker}(\varphi) x, \operatorname{Ker}(\varphi) y \in L / \operatorname{Ker}(\varphi)$ tais que $\psi(\operatorname{Ker}(\varphi) x)=\psi(\operatorname{Ker}(\varphi) y)$. Assim $\varphi(x)=\varphi(y)$. Como $x, y \in L$, existe $z \in L$ tal que $y=z * x$. Logo:

$$
\varphi(y)=\varphi(z * x)=\varphi(z) \cdot \varphi(x)=\varphi(z) \cdot \varphi(y)
$$

Pela lei do cancelamento, segue que $\varphi(z)=1_{L^{\prime}}$, e assim $z \in \operatorname{Ker}(\varphi)$. Logo $\operatorname{Ker}(\varphi) x=\operatorname{Ker}(\varphi) y$, e segue que $\psi$ é injetora.

(iv.4) $\psi$ é homomorfismo.

Sejam $\operatorname{Ker}(\varphi) x, \operatorname{Ker}(\varphi) y \in L / \operatorname{Ker}(\varphi)$. Assim:

$\psi(\operatorname{Ker}(\varphi) x * \operatorname{Ker}(\varphi) y)=\psi(\operatorname{Ker}(\varphi)(x * y))=\varphi(x * y)=\varphi(x) \cdot \varphi(y)=\psi(\operatorname{Ker}(\varphi) x) \cdot \psi(\operatorname{Ker}(\varphi) y)$

Logo $\psi$ é homomorfismo.

Portanto, pelos itens (iv.1) a (iv.4), temos que $L / \operatorname{Ker}(\varphi) \cong \operatorname{Im}(\varphi)$. 


\subsection{Loop potencialmente associativo}

Seja $S$ um subconjunto não vazio de um loop $L$. Denotamos por $\langle S\rangle$ o menor subloop de $L$ que contém o conjunto $S$, ou seja, ele tem a propriedade de que todo subloop de $L$ que contém $S$ também o contém. Com isso, temos a seguinte expressão para $\langle S\rangle$ :

$$
\langle S\rangle=\bigcap_{H \leq L, S \subseteq H} H
$$

Chamamos $\langle S\rangle$ o subloop gerado por $S$. No caso onde $S$ possui um único elemento $x$ pertencente a $L$, denotamos $\langle S\rangle$ por $\langle x\rangle$, e o chamamos de subloop gerado por $x$.

Definição 2.3.1. Um loop $L$ é dito ser potencialmente associativo se, para todo $x$ pertencente a $L$, temos que $\langle x\rangle$ é associativo.

A partir desta definição, devido a propriedade de que $\langle x\rangle$ é grupo, temos o seguinte resultado para loops potencialmente associativos:

Proposição 2.3.2. Seja $L$ um loop potencialmente associativo. Então todo elemento de L possui inverso bilateral.

Agora, para $x$ pertencente a um loop $(L, *)$, defina as potências de $x$ como segue:

$x^{0}=1$

$x^{n+1}=x^{n} * x$ para $n$ pertencente a $\mathbb{N}$

Observe que $\langle x\rangle$ contém todas as potências de $x$.

No caso onde $L$ possui a propriedade de que todo elemento possui inverso bilateral, podemos também definir as potências negativas de cada elemento $x$ como sendo $x^{-n}=\left(x^{n}\right)^{-1}$. Note que, neste caso, $\langle x\rangle$ contém também todas as potências negativas de $x$. Com estas definições, temos o resultado seguinte.

Proposição 2.3.3. Seja L um loop onde todo elemento possui inverso bilateral. Então L é potencialmente associativo se, e somente se, para todo $x$ pertencente a $L$ e para todo $n, r$ e s pertencentes $a \mathbb{Z}$ temos

$$
\left(x^{n} * x^{r}\right) * x^{s}=x^{n} *\left(x^{r} * x^{s}\right)
$$

Demonstração. Nesta demonstração denotaremos hipótese de indução por HI.

$(=>)$ Decorre do fato de $\langle x\rangle$ ser associativo e conter todas as potências de $x$.

$(<=)$ Fixado $x \in L$, primeiro vamos provar os itens de (i) a (vi) a seguir. Omitiremos os parenteses quando a equação estiver na forma 2.4 .

(i) $x^{n+m}=x^{n} * x^{m}$ para todo $n, m \in \mathbb{N}$

Fixado $n \in \mathbb{N}$, seja a nossa hipótese de indução sobre $m$ que $x^{n+m}=x^{n} * x^{m}$. Para $m=0$, temos que a hipótese é válida. Agora, suponha que ela é válida para $m$. Assim:

$$
x^{n+(m+1)}=x^{n+m} * x \stackrel{\mathrm{HI}}{=} x^{n} * x^{m} * x=x^{n} * x^{m+1}
$$


(ii) $\left(x^{n} * x^{m}\right)^{-1}=x^{-m} * x^{-n}$ para todo $n, m \in \mathbb{N}$

Fixados $n, m \in \mathbb{N}$ temos:

$$
\left(x^{n} * x^{m}\right) *\left(x^{-m} * x^{-n}\right) \stackrel{(i)}{=} x^{n+m} * x^{-m} * x^{-n} \stackrel{(i)}{=}\left(x^{n} * x^{m} * x^{-m}\right) * x^{-n}=x^{n} * x^{-n}=1
$$

(iii) $x * x^{r}=x^{r} * x$ para todo $r \in \mathbb{Z}$

Primeiramente, vamos mostrar que $x * x^{n}=x^{n} * x$ para todo $n \in \mathbb{N}$. Seja a nossa hipótese de indução que $x * x^{n}=x^{n} * x$. Para $n=0$, temos que a hipótese é válida. Agora suponha que ela é válida para $n$. Assim:

$$
x * x^{n+1}=x * x^{n} * x \stackrel{\mathrm{HI}}{=} x^{n} * x * x=x^{n+1} * x
$$

Agora, vamos mostrar que $x * x^{-n}=x^{-n} * x$ para todo $n \in \mathbb{N}$. Seja a nossa hipótese de indução que $x * x^{-n}=x^{-n} * x$. Para $n=0$, temos que a hipótese é válida. Suponha que ela é válida para n. Assim:

$x * x^{-(n+1)}=x *\left(x^{n+1}\right)^{-1}=x *\left(x^{n} * x\right)^{-1} \stackrel{(\mathrm{ii})}{=} x * x^{-1} * x^{-n}=x^{-1} * x * x^{-n} \stackrel{\mathrm{HI}}{=} x^{-1} * x^{-n} * x=$

$\stackrel{\text { (ii) }}{=}\left(x^{n+1}\right)^{-1} * x=x^{-(n+1)} * x$

(iv) $x^{-n}=\left(x^{-1}\right)^{n}$ para todo $n \in \mathbb{N}$

Seja a nossa hipótese de indução que $x^{-n}=\left(x^{-1}\right)^{n}$. Para $n=0$, temos que a hipótese é válida. Agora suponha que ela é válida para $n$. Assim:

$$
x^{n+1} *\left(x^{-1}\right)^{n+1}=x^{n+1} *\left(x^{-1}\right)^{n} * x^{-1} \stackrel{(\mathrm{iii})}{=}\left(x * x^{n} *\left(x^{-1}\right)^{n}\right) * x^{-1} \stackrel{H I}{=}\left(x * x^{n} * x^{-n}\right) * x^{-1}=x * x^{-1}=1
$$

(v) $x^{r+1}=x^{r} * x$ para todo $r \in \mathbb{Z}$

Para $r>0$ a afirmação é válida por definição. Assim basta provarmos que $x^{-n+1}=x^{-n} * x$, para todo $n \in \mathbb{N}$. Seja a nossa hipótese de indução que $x^{-n+1}=x^{-n} * x$. Para $n=0$, temos que a hipótese é válida. Também é fácil ver que ela é válida para $n=1$. Agora suponha que ela é válida para $n, \operatorname{com} n>0$. Assim:

$$
\begin{aligned}
& x^{-(n+1)+1}=x^{-n}=\left(x^{n}\right)^{-1} \stackrel{n \geq 0}{=}\left(x^{n-1} * x\right)^{-1} \stackrel{(\mathrm{ii}), n>0}{=} x^{-1} * x^{-n+1} \stackrel{(\mathrm{HI})}{=} x^{-1} * x^{-n} * x \stackrel{(\mathrm{iii})}{=} x^{-1} * x * x^{-n}= \\
& =x * x^{-1} * x^{-n} \stackrel{(\mathrm{ii})}{=} x *\left(x^{n} * x\right)^{-1}=x *\left(x^{n+1}\right)^{-1}=x * x^{-n-1} \stackrel{(\mathrm{iii})}{=} x^{-(n+1)} * x
\end{aligned}
$$

(vi) $x^{r+s}=x^{r} * x^{s}$ para todo $r, s \in \mathbb{Z}$

Fixado $r \in \mathbb{Z}$, primeiramente vamos mostrar que $x^{r+n}=x^{r} * x^{n}$, para todo $n \in \mathbb{N}$. Seja a nossa hipótese de indução que $x^{r+n}=x^{r} * x^{n}$. Para $n=0$, temos que a hipótese é válida. Agora suponha que ela é válida para $n$. Assim:

$$
x^{r+(n+1)} \stackrel{(\mathrm{v})}{=} x^{r+n} * x \stackrel{\mathrm{HI}}{=} x^{r} * x^{n} * x=x^{r} * x^{n+1}
$$

Agora, vamos mostrar que $x^{r-n}=x^{r} * x^{-n}$, para todo $n \in \mathbb{N}$. Seja a nossa hipótese de indução que $x^{r-n}=x^{r} * x^{-n}$. Para $n=0$, temos que a hipótese é válida. Agora suponha que ela é válida para n. Assim: 
$x^{r} * x^{-(n+1)} \stackrel{(\mathrm{iv})}{=} x^{r} *\left(x^{-1}\right)^{(n+1)}=x^{r} * x^{-n} * x^{-1} \stackrel{\mathrm{HI}}{=} x^{r-n} * x^{-1} \stackrel{\mathrm{v}}{=} x^{r-n-1} * x * x^{-1}=x^{r-(n+1)}$

(vii) Agora, seja $H=\left\{x^{n} \mid n \in \mathbb{Z}\right\}$. Da equação 2.4 segue que $H$ é associativo. Se $H$ for subloop, da definição de $\langle x\rangle$ segue que ele está contido em $H$, sendo assim associativo. Assim basta mostrarmos que $H$ é subloop de $L$.

Fixados $x^{r}, x^{s} \in H, \operatorname{com} r, s \in \mathbb{Z}$, de (vi) segue que $x^{r} * x^{s}=x^{r+s}, x^{r-s} * x^{s}=x^{r}$ e $x^{s} * x^{r-s}=x^{r}$. Como $x^{r+s}$ e $x^{r-s}$ pertencem a $H$, da proposição 2.2.2 segue que $H$ é subloop de $L$.

Para o caso onde $L$ é finito, podemos retirar algumas condições da proposição anterior, obtendo o resultado seguinte.

Proposição 2.3.4. Seja L um loop finito. Então L é potencialmente associativo se, e somente se, para todo $x$ pertencente a $L$ e para todo $n, r$ e $s$ pertencentes a $\mathbb{N}$ temos

$$
\left(x^{n} * x^{r}\right) * x^{s}=x^{n} *\left(x^{r} * x^{s}\right)
$$

Demonstração. Nesta demonstração denotaremos hipótese de indução por HI.

$(=>)$ Decorre do fato de $\langle x\rangle$ ser associativo e conter todas as potências positivas de $x$.

$(<=)$ Fixado $x \in L$, primeiramente vamos mostrar que $x^{n+m}=x^{n} * x^{m}$ para todo $n, m \in \mathbb{N}$.

Fixado $n \in \mathbb{N}$, seja a nossa hipótese de indução sobre $m$ que $x^{n+m}=x^{n} * x^{m}$. Para $m=0$, temos que a hipótese é válida. Agora suponha que ela é válida para $m$. Assim:

$$
x^{n+(m+1)}=x^{n+m} * x \stackrel{\text { HI }}{=}\left(x^{n} * x^{m}\right) * x \stackrel{\text { eq. } 2.5}{=} x^{n} *\left(x^{m} * x\right)=x^{n} * x^{m+1}
$$

Portanto, $x^{n+m}=x^{n} * x^{m}$ para todo $n, m \in \mathbb{N}$.

Agora, defina $H=\left\{x^{n} \mid n \in \mathbb{N}\right\}$. Da equação 2.5 segue que $H$ é associativo. Fixados $x^{r}, x^{s} \in H$, temos que $x^{r} * x^{s}=x^{r+s} \in H$. Logo, da proposição 2.2.3 segue que $H$ é subloop de $L$. Portanto, da definição de $\langle x\rangle$ segue que ele está contido em $H$ e assim é associativo.

Para $x$ pertencente a um loop $L$, definimos a ordem de $x$ como sendo a ordem do subloop $\langle x\rangle$, e a denotamos por $o(x)$. Da teoria de grupos segue o seguinte resultado:

Proposição 2.3.5. Seja $L$ um loop potencialmente associativo e $x$ pertencente a $L$ tal que o(x) é finita. Então o $(x)$ é o menor número natural $n$ tal que $x^{n}=1$. Além disso, se para $m$ pertencente $a \mathbb{N}$ temos $x^{m}=1$, então o $(x)$ divide $m$.

\subsection{Loops de Moufang}

Definição 2.4.1. Dizemos que um loop é de Moufang se satisfaz qualquer uma das três identidades a seguir.

(1) $((x * y) * x) * z=x *(y *(x * z))$,

(identidade à esquerda de Moufang)

(2) $((x * y) * z) * y=x *(y *(z * y))$, (identidade à direita de Moufang)

(3) $(x * y) *(z * x)=(x *(y * z)) * x$,

(identidade do meio de Moufang) 
Proposição 2.4.2. ([GJM96] Teorema 3.1) Em qualquer loop, as três identidades acima são equivalentes.

Convém observar que, mesmo com a falta da associatividade, muitas propriedades de grupo ainda são válidas para loops de Moufang.

Um loop é diassociativo se para quaisquer dois de seus elementos, o subloop gerado por eles é um grupo. Este é o caso para loops de Moufang, que além disso possuem uma propriedade mais forte, como pode ser observado no teorema de Moufang, enunciado a seguir.

Teorema 2.4.3. (Teorema de Moufang) Se a,b e c são elementos de um loop de Moufang L que se associam em alguma ordem, então o subloop gerado por a, b e c é um subgrupo de $L$.

A demonstração do teorema de Moufang pode ser encontrada no livro de H.O. Pflugfelder [Pf190] ou em [Drá10] onde A. Drápal apresenta uma demonstração mais moderna.

\subsection{Loops automórficos}

Definição 2.5.1. Dizemos que um loop $L$ é um loop automórfico, ou simplesmente $A$-loop, se todos os elementos de $\operatorname{Inn}(L)$ são automorfismos de $L$.

Observe que da proposição 2.1.4 segue que um loop $L$ é $A$-loop se, e somente se, as bijeções $T_{x}, L_{(x, y)}$ e $R_{(x, y)}$ são automorfismos de $L$, para todo $x$ e $y$ pertencentes a $L$.

Exemplo 2.5.2. Seja $L=\{1,2,3,4,5,6\}$ e defina em $L$ a operação $*$ dada pela seguinte tabela de multiplicação:

\begin{tabular}{|c|c|c|c|c|c|c|}
\hline$*$ & 1 & 2 & 3 & 4 & 5 & 6 \\
\hline 1 & 1 & 2 & 3 & 4 & 5 & 6 \\
\hline 2 & 2 & 3 & 1 & 6 & 4 & 5 \\
\hline 3 & 3 & 1 & 2 & 5 & 6 & 4 \\
\hline 4 & 4 & 5 & 6 & 1 & 3 & 2 \\
\hline 5 & 5 & 6 & 4 & 2 & 1 & 3 \\
\hline 6 & 6 & 4 & 5 & 3 & 2 & 1 \\
\hline
\end{tabular}

Usando o software GAP [Gro], é simples verificar que $(L, *)$ é um $A$-loop. Além disso, este é o menor $A$-loop não associativo.

Um subloop $H$ de um loop $L$ é dito ser característico se $\varphi(H)=H$, para todo automorfismo $\varphi$ de $L$. Na teoria de grupos temos que todo subgrupo característico é também subgrupo normal e, embora esta condição não é válida para loops em geral, ela é válida para $A$-loops. Este e outros resultados básicos para $A$-loops foram provados por R.H. Bruck e L.J. Paige [BP56] e são apresentados na proposição abaixo:

Proposição 2.5.3. ([BP56]) Seja L um A-loop. Então:

a) Se $H$ é subloop de L, então $H$ é A-loop

b) $S e H \triangleleft L$, então $L / H$ é A-loop

c) $L$ é potencialmente associativo

d) L tem a propriedade flexível, ou seja, $x(y x)=(x y) x$, para todo $x$ e y pertencentes a $L$

e) Se $H$ é subloop característico de $L$, então $H \triangleleft L$

f) $N_{\mu}(L)$ está contido em $N_{\lambda}(L)$ e em $N_{\rho}(L)$ 
A proposição a seguir complementa os resultados anteriores e podem ser encontrada no artigo [KKPV16].

Proposição 2.5.4. ([KKPV16]) Seja L um A-loop. Então:

a) $(x y)^{-1}=y^{-1} x^{-1}$, para todo $x$ e y pertencentes a $L$

b) $(a * x) * b=a *(x * b)$, para todo a e $b$ pertencentes a $N_{\mu}(L)$ e para todo $x$ pertencente $a L$

c) $N(L)=N_{\lambda}(L)=N_{\rho}(L)$

d) $\operatorname{Inn}(L)=\left\langle L_{(x, y)}, T_{x} \mid x, y \in L\right\rangle$

e) (Teorema de Lagrange) Se a ordem de $L$ é ímpar e $H$ é subloop de $L$, então $|H|$ divide $|L|$

f) (Teorema de Cauchy) Se a ordem de L é ímpar e p é um primo que divide a ordem de L, então existe $x$ pertencente a $L$ tal que $o(x)=p$.

Seja $L$ um loop e $S$ um subconjunto não vazio de $L$. Definimos o centralizador do subconjunto $S$ em $L$, o centralizador do elemento $x$ em $L$ e o comutante de $L$, denotados respectivamente por $C_{L}(x)$ e $C(L)$, como segue:

$C_{L}(S)=\{y \in L \mid y s=s y \quad \forall s \in S\}$

$C_{L}(x)=\{y \in L \mid x y=y x\}$

$C(L)=\{y \in L \mid y z=z y \quad \forall z \in L\}$

Note que os conjuntos $C_{L}(x)$ e $C(L)$ são respectivamente os centralizadores dos subconjuntos $\{x\}$ e $L$.

Os conjuntos $C_{L}(S), C_{L}(x)$ e $C(L)$ não são necessariamente subloops para loops em geral, mas o são para $A$-loops. Este resultado segue imediatamente do seguinte lema:

Lema 2.5.5. ([BP56] Lema 2.3) Sejam L um loop, $\Omega$ um conjunto não vazio de automorfismos de $L$ e Fix $(\Omega)$ definido como segue:

$$
\operatorname{Fix}(\Omega)=\{x \in L \mid \phi(x)=x \quad \forall \phi \in \Omega\}
$$

Então Fix $(\Omega)$ é subloop de L.

Desde que $C_{L}(S)=F i x\left(\left\{T_{s} \mid s \in S\right\}\right)$ podemos então estabelecer o resultado abaixo.

Proposição 2.5.6. Sejam $L$ um A-loop, $S$ um subconjunto não vazio de $L$ e x pertencente a $L$. Então $C_{L}(S), C_{L}(x)$ e $C(L)$ são subloops de $L$. 


\section{Capítulo 3}

\section{Half-isomorfismos de loops}

Neste capítulo, introduzimos o conceito de half-isomorfismo entre loops e suas propriedades. Muitos conceitos e também algumas propriedades presentes aqui são inéditos e foram desenvolvidos durante esse trabalho.

\subsection{Definição e exemplo}

Definição 3.1.1. Sejam $(L, *)$ e $\left(L^{\prime}, \cdot\right)$ loops. A função $f: L \rightarrow L^{\prime}$ é um half-isomorfismo se $f$ é bijetora e para todo $x$ e $y$ pertencentes a $L$ :

$$
f(x * y) \in\{f(x) \cdot f(y), f(y) \cdot f(x)\}
$$

No caso em que $L^{\prime}=L$ e $f$ satisfaz as condições anteriores, dizemos que $f$ é um halfautomorfismo.

Uma função bijetora $f: L \rightarrow L^{\prime}$ é dita ser um isomorfismo se $f(x * y)=f(x) \cdot f(y)$, para todo $x$ e $y$ pertencentes a $L$, e é dita ser um anti-isomorfismo se $f(x * y)=f(y) \cdot f(x)$, para todo $x$ e $y$ pertencentes a $L$. Observe que todo isomorfismo e todo anti-isomorfismo são também half-isomorfismos.

Dizemos que um half-isomorfismo $f: L \rightarrow L^{\prime}$ é trivial quando $f$ é um isomorfismo ou um anti-isomorfismo.

Observação 3.1.2. De 3.1, segue que um half-isomorfismo $f: L \rightarrow L^{\prime}$ satisfaz, para todo $x$ e $y$ pertencentes a $L$ :

$$
\{f(x * y), f(y * x)\} \subseteq\{f(x) \cdot f(y), f(y) \cdot f(x)\}
$$

A seguir será dado um exemplo de half-isomorfismo não trivial entre loops. As tabelas de multiplicação e a função dadas nele foram obtidas através de programação feita no software GAP [Gro].

Exemplo 3.1.3. Considere o loop automórfico não associativo $L=\{1,2, \ldots, 8\}$ cuja a tabela de multiplicação é dada abaixo. 


\begin{tabular}{|l|l|l|l|l|l|l|l|l|}
\hline$*$ & 1 & 2 & 3 & 4 & 5 & 6 & 7 & 8 \\
\hline 1 & 1 & 2 & 3 & 4 & 5 & 6 & 7 & 8 \\
\hline 2 & 2 & 1 & 4 & 3 & 6 & 5 & 8 & 7 \\
\hline 3 & 3 & 4 & 1 & 2 & 7 & 8 & 6 & 5 \\
\hline 4 & 4 & 3 & 2 & 1 & 8 & 7 & 5 & 6 \\
\hline 5 & 5 & 6 & 8 & 7 & 1 & 2 & 4 & 3 \\
\hline 6 & 6 & 5 & 7 & 8 & 2 & 1 & 3 & 4 \\
\hline 7 & 7 & 8 & 5 & 6 & 3 & 4 & 2 & 1 \\
\hline 8 & 8 & 7 & 6 & 5 & 4 & 3 & 1 & 2 \\
\hline
\end{tabular}

Seja $f$ a permutação $(35)(46)$ de $L$. Usando o software GAP é simples verificar que $f$ é um half-automorfismo. Além disso, $f$ é um half-automorfismo não trivial, como podemos ver abaixo:

$$
\begin{array}{lll}
f(3 * 7)=f(6)=4 & f(3) * f(7)=5 * 7=4 & f(7) * f(3)=7 * 5=3 \\
f(3 * 5)=f(7)=7 & f(3) * f(5)=5 * 3=8 & f(5) * f(3)=3 * 5=7
\end{array}
$$

\subsection{Propriedades}

Proposição 3.2.1. Sejam $f: L \rightarrow L^{\prime}$ um half-isomorfismo e $H$ um subloop de $L^{\prime}$. Então:

a) $f^{-1}(H)$ é subloop de $L$.

b) Se $H$ é comutativo, então $f^{-1}(H)$ é comutativo e $f^{-1}(H)$ é isomorfo a $H$.

c) Se $H$ é grupo abeliano, então $f^{-1}(H)$ é grupo abeliano.

d) Se $L^{\prime}$ é comutativo, então $L$ é isomorfo a $L^{\prime}$.

Demonstração. a) Sejam $x, y \in f^{-1}(H)$. Então $f(x), f(y) \in H$, e assim $\{f(x) \cdot f(y), f(y) \cdot f(x)\} \subseteq H$. Logo, da equação 3.2 , segue que $f(x * y), f(y * x) \in H$, e assim $x * y \in f^{-1}(H)$.

Agora sejam $w, z \in L$ tais que $x * w=y$ e $z * x=y$. Da equação 3.1, segue que:

$f(y) \in\{f(x) \cdot f(w), f(w) \cdot f(x)\}$

$f(y) \in\{f(x) \cdot f(z), f(z) \cdot f(x)\}$

Assim, como $f(x), f(y) \in H$, segue que $f(w), f(z) \in H$. Logo temos que $w, z \in f^{-1}(H)$, e portanto $f^{-1}(H)$ é subloop de $L$.

b) Sejam $x, y \in f^{-1}(H)$. Usando a equação 3.2:

$$
\{f(x * y), f(y * x)\} \subseteq\{f(x) \cdot f(y), f(y) \cdot f(x)\} \stackrel{H \text { comut. }}{=}\{f(x) \cdot f(y)\}
$$

Assim, $f(x * y)=f(y * x)=f(x) \cdot f(y)$. Como $f$ é bijetora, segue que $x * y=y * x, \log$ o $f^{-1}(H)$ é comutativo. Além disso, temos que $f(x * y)=f(x) \cdot f(y)$ para todo $x, y \in f^{-1}(H)$, e $\operatorname{assim} f^{-1}(H) \cong H$.

Os itens c) e d) decorrem de b).

Lema 3.2.2. Seja $f: L \rightarrow L^{\prime}$ um half-isomorfismo, e $L$ e $L^{\prime}$ com identidades $1_{L}$ e $1_{L^{\prime}}$. Então $f\left(1_{L}\right)=1_{L^{\prime}}$. 
Demonstração. Aplicando $f$ em $1_{L}$, temos que $f\left(1_{L}\right)=f\left(1_{L} * 1_{L}\right)=f\left(1_{L}\right) \cdot f\left(1_{L}\right)$. Portanto, $f\left(1_{L}\right)=1_{L^{\prime}}$

Proposição 3.2.3. Sejam $f: L \rightarrow L^{\prime}$ um half-isomorfismo e $L^{\prime}$ com a propriedade de que todo elemento possui inverso bilateral. Então todo elemento $x$ de $L$ possui inverso bilateral $x^{-1}=f^{-1}\left((f(x))^{-1}\right)$.

Demonstração. Fixado $x \in L$, como $f(x) \in L^{\prime}$, temos que existe $(f(x))^{-1} \in L^{\prime}$. Logo, da equação 3.2 :

$$
\left\{f\left(x * f^{-1}\left((f(x))^{-1}\right)\right), f\left(f^{-1}\left((f(x))^{-1}\right) * x\right)\right\} \subseteq\left\{f(x) \cdot(f(x))^{-1},(f(x))^{-1} \cdot f(x)\right\}=\left\{1_{L^{\prime}}\right\}
$$

Logo $f\left(x * f^{-1}\left((f(x))^{-1}\right)\right)=f\left(f^{-1}\left((f(x))^{-1}\right) * x\right)=1_{L^{\prime}}$. Assim do lema 3.2.2 e do fato de $f$ ser bijetora, segue que $x * f^{-1}\left((f(x))^{-1}\right)=f^{-1}\left((f(x))^{-1}\right) * x=1_{L}$. Portanto, $x^{-1}=f^{-1}\left((f(x))^{-1}\right)$.

Proposição 3.2.4. Sejam $f: L \rightarrow L^{\prime}$ um half-isomorfismo e $L^{\prime}$ potencialmente associativo. Então:

a) $f\left(x^{n}\right)=(f(x))^{n}$, para todo $x$ pertencente a $L$ e para todo $n$ pertencente $a \mathbb{Z}$.

b) L é potencialmente associativo.

c) Se $x$ pertencente a L é tal que o(f(x)) é finita, então o $(x)=o(f(x))$.

Demonstração. a) Seja $x \in L$. Pelo lema 3.2.2 temos que $f\left(x^{0}\right)=(f(x))^{0}$.

Suponha que $f\left(x^{n}\right)=(f(x))^{n}$ para $n \in \mathbb{N}$. Assim:

$$
f\left(x^{n+1}\right)=f\left(x^{n} * x\right) \in\left\{(f(x))^{n} \cdot f(x), f(x) \cdot(f(x))^{n}\right\} \stackrel{L^{\prime}}{\text { pot. ass. }}\left\{(f(x))^{n+1}\right\}
$$

Portanto $f\left(x^{n}\right)=(f(x))^{n}$, para todo $n \in \mathbb{N}$.

Agora, como $L^{\prime}$ é potencialmente associativo, da proposição 2.3.2 temos que todo elemento de $L^{\prime}$ possui inverso bilateral, assim da proposição 3.2.3 segue que todo elemento de $L$ possui inverso bilateral. Desta forma, para $n \in \mathbb{N}$ temos:

$$
x^{-n}=\left(x^{n}\right)^{-1} \stackrel{\text { prop } 3.2 .3}{=} f^{-1}\left(f\left(x^{n}\right)^{-1}\right)
$$

Logo $f\left(x^{-n}\right)=f\left(x^{n}\right)^{-1}=\left((f(x))^{n}\right)^{-1}=(f(x))^{-n}$.

b) Sejam $x \in L$ e $n, r, s \in \mathbb{Z}$. Então:

$$
\begin{aligned}
& \left\{f\left(\left(x^{n} * x^{r}\right) * x^{s}\right), f\left(x^{n} *\left(x^{r} * x^{s}\right)\right)\right\} \subseteq\left\{\left(f(x)^{n} \cdot f(x)^{r}\right) \cdot f(x)^{s}, f(x)^{n} \cdot\left(f(x)^{r} \cdot f(x)^{s}\right), \ldots\right. \\
\ldots, & \left.\left(f(x)^{s} \cdot f(x)^{r}\right) \cdot f(x)^{n}\right\} \stackrel{L^{\prime} \text { pot. ass. }}{=}\left\{(f(x))^{n+r+s}\right\}
\end{aligned}
$$

Com isto, temos que $f\left(\left(x^{n} * x^{r}\right) * x^{s}\right)=f\left(x^{n} *\left(x^{r} * x^{s}\right)\right)$, e como $f$ é bijetora, segue que $\left(x^{n} * x^{r}\right) * x^{s}=x^{n} *\left(x^{r} * x^{s}\right)$. Portanto da proposição 2.3.3, temos que $L$ é potencialmente associativo.

c) Seja $x \in L$ tal que $o(f(x))<\infty$. De a) temos $f\left(x^{o(f(x))}\right)=(f(x))^{o(f(x))}=1_{L^{\prime}}$. Como $f$ é bijetora, segue que $x^{o(f(x))}=1_{L}$, logo $o(x) \leq o(f(x))<\infty$. Assim, usando a) novamente temos $(f(x))^{o(x)}=f\left(x^{o(x)}\right)=f\left(1_{L}\right)=1_{L^{\prime}}, \log o o(f(x)) \leq o(x)$. 
Defina o seguinte conjunto:

$$
K(L)=\{(x, y) \in L \times L \mid x * y=y * x\}
$$

Proposição 3.2.5. Sejam $f: L \rightarrow L^{\prime}$ um half-isomorfismo e $K(L)$ finito. Então $\left|K\left(L^{\prime}\right)\right|$ é menor ou igual à $|K(L)|$.

Demonstração. Como $f$ é bijeção, o conjunto $K\left(L^{\prime}\right)$ pode ser reescrito na seguinte forma:

$$
K\left(L^{\prime}\right)=\left\{(f(x), f(y)) \in L^{\prime} \times L^{\prime} \mid f(x) \cdot f(y)=f(y) \cdot f(x), x, y \in L\right\}
$$

Assim, seja $(f(x), f(y)) \in K\left(L^{\prime}\right), \operatorname{com} x, y \in L$. Logo, $f(x) \cdot f(y)=f(y) \cdot f(x)$. De 3.2 temos:

$$
\{f(x * y), f(y * x)\} \subseteq\{f(x) \cdot f(y), f(y) \cdot f(x)\}=\{f(x) \cdot f(y)\}
$$

Com isto, $f(x * y)=f(y * x)$, e como $f$ é bijetora, segue que $x * y=y * x$. Assim $(x, y) \in K(L)$. Logo a função:

$$
\psi: \begin{array}{ccc}
K\left(L^{\prime}\right) & \rightarrow & K(L) \\
(f(x), f(y)) & \mapsto & (x, y)
\end{array}
$$

está bem definida. Além disso, como $f$ é bijetora, segue que $\psi$ é injetora. Portanto $\left|K\left(L^{\prime}\right)\right| \leq|K(L)|$.

Definição 3.2.6. Um half-isomorfismo $f: L \rightarrow L^{\prime}$ é dito ser especial se a função $f^{-1}: L^{\prime} \rightarrow L$ também for um half-isomorfismo.

Teorema 3.2.7. Seja $f: L \rightarrow L^{\prime}$ um half-isomorfismo. São equivalentes:

a) $f$ é especial.

b) $\{f(x * y), f(y * x)\}=\{f(x) \cdot f(y), f(y) \cdot f(x)\}$, para todo $x$ e y pertencentes a $L$.

c) Para todo $x$ e y pertencentes a $L$ tais que $x * y=y * x$, temos $f(x) \cdot f(y)=f(y) \cdot f(x)$.

Além disso, no caso em que $K(L)$ é finito, estas condições são equivalentes à:

d) $|K(L)|=\left|K\left(L^{\prime}\right)\right|$

Demonstração. a) $=>$ b) Sejam $x, y \in L$. Como $f$ é half-isomorfismo, da equação 3.2 temos:

$$
\{f(x * y), f(y * x)\} \subseteq\{f(x) \cdot f(y), f(y) \cdot f(x)\}
$$

Como $f^{-1}$ é half-isomorfismo, da equação 3.2 temos:

$$
\left\{f^{-1}(f(x) \cdot f(y)), f^{-1}(f(y) \cdot f(x))\right\} \subseteq\{x * y, y * x\}
$$

Logo, $\{f(x) \cdot f(y), f(y) \cdot f(x)\} \subseteq\{f(x * y), f(y * x)\}$.

b) $=>$ a) Sejam $f(x), f(y) \in L^{\prime}$. Como $\{f(x * y), f(y * x)\}=\{f(x) \cdot f(y), f(y) \cdot f(x)\}$, aplicando $f^{-1}$ nesta igualdade, temos:

$$
\{x * y, y * x\}=\left\{f^{-1}(f(x) \cdot f(y)), f^{-1}(f(y) \cdot f(x))\right\}
$$

Logo $f^{-1}(f(x) \cdot f(y)) \in\left\{f^{-1}(f(x)) * f^{-1}(f(y)), f^{-1}(f(y)) * f^{-1}(f(x))\right\}$.

b) $=>$ c) Sejam $x, y \in L$ tais que $x * y=y * x$. Como $f$ é bijetora, segue que $f(x * y)=f(y * x)$. Assim, da hipótese segue que: 


$$
\{f(x) \cdot f(y), f(y) \cdot f(x)\}=\{f(x * y), f(y * x)\}=\{f(x * y)\}
$$

Logo $f(x) \cdot f(y)=f(y) \cdot f(x)$.

c) $=>$ b) Sejam $x, y \in L$. Temos dois casos:

(i) Suponha que $x * y=y * x$. Como $f$ é bijetora, segue que $f(x * y)=f(y * x)$, e da hipótese temos que $f(x) \cdot f(y)=f(y) \cdot f(x)$. Logo da definição de half-isomorfismo, segue que $f(x * y)=f(x) \cdot f(y)$, e desta forma:

$$
\{f(x * y), f(y * x)\}=\{f(x * y)\}=\{f(x) \cdot f(y)\}=\{f(x) \cdot f(y), f(y) \cdot f(x)\}
$$

(ii) Suponha que $x * y \neq y * x$. Como $f$ é bijetora, segue que $f(x * y) \neq f(y * x)$. De 3.2, temos

$$
\{f(x * y), f(y * x)\} \subseteq\{f(x) \cdot f(y), f(y) \cdot f(x)\}
$$

Assim, do fato do conjunto da esquerda ter dois elementos e o da direita ter no máximo dois, segue a igualdade.

a) => d) Como $|K(L)|<\infty$, da proposição 3.2.5 segue que $\left|K\left(L^{\prime}\right)\right| \leq|K(L)|<\infty$. Logo, como $f^{-1}: L^{\prime} \rightarrow L$ é um half-isomorfismo, da proposição 3.2 .5 segue que $|K(L)| \leq\left|K\left(L^{\prime}\right)\right|$.

d) => c) Pela demonstração da proposição 3.2.5, segue que a função $\psi: K\left(L^{\prime}\right) \rightarrow K(L)$, com $\psi((f(x), f(y)))=(x, y)$, é injetora. Como $|K(L)|=\left|K\left(L^{\prime}\right)\right|$, segue que $\psi$ é bijetora. Assim, sejam $x, y \in L$ tais que $x * y=y * x$. Logo $(x, y) \in K(L)$, e como $\psi$ é bijetora, segue que $(f(x), f(y)) \in K\left(L^{\prime}\right)$, e portanto $f(x) \cdot f(y)=f(y) \cdot f(x)$.

Do item d) do teorema 3.2.7, seguem os corolários a seguir, onde identificamos condições para que todo half-automorfismo do loop seja especial.

Corolário 3.2.8. Seja $L$ um loop tal que $K(L)$ é finito. Então todo half-automorfismo de L é especial.

Corolário 3.2.9. Seja L loop finito. Então todo half-automorfismo de L é especial.

No caso onde temos um half-isomorfismo especial, podemos usar a função inversa do halfisomorfismo nas proposições 3.2.1, 3.2.3 e 3.2.4, obtendo assim recíprocas das mesmas. Com isso, na proposição a seguir estão reunidos os resultados válidos para half-isomorfismos especiais.

Proposição 3.2.10. Sejam $f: L \rightarrow L^{\prime}$ um half-isomorfismo especial e $H$ subloop de L. Então:

a) $f(H)$ é subloop de $L^{\prime}$.

b) Se $H$ é comutativo, então $f(H)$ é comutativo e $f(H)$ é isomorfo a $H$.

c) Se $H$ é grupo abeliano, então $f(H)$ é grupo abeliano.

d) Se $L$ é comutativo, então $L$ é isomorfo a $L^{\prime}$.

e) Se L tem a propriedade de que todo elemento possui inverso bilateral, então todo elemento $x$ de

$L^{\prime}$ possui inverso bilateral $x^{-1}=f\left(\left(f^{-1}(x)\right)^{-1}\right)$.

f) Se $L$ é potencialmente associativo, então $f\left(x^{n}\right)=(f(x))^{n}$, para todo $x$ pertencente a $L$ e para todo $n$ pertencente a $\mathbb{Z}$.

g) Se L é potencialmente associativo, então $L^{\prime}$ é potencialmente associativo.

h) Se $L$ é potencialmente associativo e x pertencente a $L$ é tal que o $(x)$ é finita, então $o(x)=o(f(x))$. 
i) $\{f(x * y), f(y * x)\}=\{f(x) \cdot f(y), f(y) \cdot f(x)\}$, para todo $x$ e y pertencentes a L.

j) Para todo $x$ e y pertencentes a $L$ tais que $x * y=y * x$, temos $f(x) \cdot f(y)=f(y) \cdot f(x)$.

k) Se $K(L)$ é finito, então $|K(L)|=\left|K\left(L^{\prime}\right)\right|$

Quando o half-isomorfismo $f$ não for especial, temos que as recíproca das proposições 3.2.1, 3.2.3 e 3.2.4 nem sempre são válidas. Nos exemplos a seguir analisaremos este caso.

Exemplo 3.2.11. Sejam $L=C_{6}$, o grupo cíclico de ordem 6 , e $f$ a função identidade. Considere as seguintes tabelas de multiplicação para os loops $L$ e $L^{\prime}$ :

\begin{tabular}{|l|l|l|l|l|l|l|}
\hline$L$ & 1 & 2 & 3 & 4 & 5 & 6 \\
\hline 1 & 1 & 2 & 3 & 4 & 5 & 6 \\
\hline 2 & 2 & 3 & 4 & 5 & 6 & 1 \\
\hline 3 & 3 & 4 & 5 & 6 & 1 & 2 \\
\hline 4 & 4 & 5 & 6 & 1 & 2 & 3 \\
\hline 5 & 5 & 6 & 1 & 2 & 3 & 4 \\
\hline 6 & 6 & 1 & 2 & 3 & 4 & 5 \\
\hline
\end{tabular}

\begin{tabular}{|c|c|c|c|c|c|c|}
\hline$L^{\prime}$ & 1 & 2 & 3 & 4 & 5 & 6 \\
\hline 1 & 1 & 2 & 3 & 4 & 5 & 6 \\
\hline 2 & 2 & 3 & 4 & 5 & 6 & 1 \\
\hline 3 & 3 & 1 & 5 & 6 & 4 & 2 \\
\hline 4 & 4 & 5 & 6 & 1 & 2 & 3 \\
\hline 5 & 5 & 6 & 1 & 2 & 3 & 4 \\
\hline 6 & 6 & 4 & 2 & 3 & 1 & 5 \\
\hline
\end{tabular}

Podemos ver que $f: L \rightarrow L^{\prime}$ é um half-isomorfismo não trivial. Note que o loop $L$ é um grupo abeliano e, portanto, comutativo, associativo, potencialmente associativo e todos os seus elementos possuem inverso bilateral. Enquanto que o loop $L^{\prime}$ é não comutativo, não associativo, não potencialmente associativo $\left(2^{2} \cdot 2=1\right.$ e $\left.2 \cdot 2^{2}=4\right)$ e existem elementos que não possuem inversos bilaterais $\left(6^{\lambda}=2,6^{\rho}=5\right)$. Também vemos que $H=\{1,3,5\}$ é subloop de $L$, enquanto $f(H)=\{1,3,5\}$ não é subloop de $L^{\prime}$. Além disso $|K(L)|=|L|^{2}$ e $\left|K\left(L^{\prime}\right)\right|$ é menor do que $|K(L)|$, logo do teorema 3.2.7 segue que $f$ não é um half-isomorfismo especial.

Exemplo 3.2.12. Agora veremos um exemplo de um half-isomorfismo não trivial entre loops de ordem ímpar. Sejam $L=C_{7}$, o grupo cíclico de ordem 7 , e $f$ a função identidade. Considere as seguintes tabelas de multiplicação para os loops $L$ e $L^{\prime}$ :

\begin{tabular}{|l|l|l|l|l|l|l|l|}
\hline$L$ & 1 & 2 & 3 & 4 & 5 & 6 & 7 \\
\hline 1 & 1 & 2 & 3 & 4 & 5 & 6 & 7 \\
\hline 2 & 2 & 3 & 4 & 5 & 6 & 7 & 1 \\
\hline 3 & 3 & 4 & 5 & 6 & 7 & 1 & 2 \\
\hline 4 & 4 & 5 & 6 & 7 & 1 & 2 & 3 \\
\hline 5 & 5 & 6 & 7 & 1 & 2 & 3 & 4 \\
\hline 6 & 6 & 7 & 1 & 2 & 3 & 4 & 5 \\
\hline 7 & 7 & 1 & 2 & 3 & 4 & 5 & 6 \\
\hline
\end{tabular}

\begin{tabular}{|c|c|c|c|c|c|c|c|}
\hline$L^{\prime}$ & 1 & 2 & 3 & 4 & 5 & 6 & 7 \\
\hline 1 & 1 & 2 & 3 & 4 & 5 & 6 & 7 \\
\hline 2 & 2 & 3 & 7 & 5 & 6 & 1 & 4 \\
\hline 3 & 3 & 4 & 5 & 6 & 7 & 2 & 1 \\
\hline 4 & 4 & 5 & 6 & 7 & 1 & 3 & 2 \\
\hline 5 & 5 & 6 & 4 & 1 & 2 & 7 & 3 \\
\hline 6 & 6 & 7 & 1 & 2 & 3 & 4 & 5 \\
\hline 7 & 7 & 1 & 2 & 3 & 4 & 5 & 6 \\
\hline
\end{tabular}

Novamente $f: L \rightarrow L^{\prime}$ é um half-isomorfismo não trivial que não preserva as propriedades de $L$ ser comutativo, associativo, potencialmente associativo e de todos os seus elementos terem inverso bilateral. Além disso, como no caso anterior, $f$ não é um half-isomorfismo especial.

Na próxima seção analisaremos o grupo de half-automorfismos de um loop. Antes disso, analisaremos a composição de half-isomorfismos.

Proposição 3.2.13. Sejam $(L, *),\left(L^{\prime}, \cdot\right)$ e $\left(L^{\prime \prime}, \bullet\right)$ loops e $f: L \rightarrow L^{\prime}$ e $g: L^{\prime} \rightarrow L^{\prime \prime}$ halfisomorfismos. Então $g \circ f: L \rightarrow L^{\prime \prime}$ é um half-isomorfismo.

Demonstração. Para $x, y \in L$, temos:

$$
f(x * y) \in\{f(x) \cdot f(y), f(y) \cdot f(x)\}
$$


Aplicando $g$ nos dois lados da equação acima, e usando o fato de que $g$ é half-isomorfismo temos:

$$
\begin{aligned}
& g(f(x * y)) \in\{g(f(x) \cdot f(y)), g(f(y) \cdot f(x))\} \subseteq \\
& \subseteq\{g(f(x)) \bullet g(f(y)), g(f(y)) \bullet g(f(x)), g(f(y)) \bullet g(f(x)), g(f(x)) \bullet g(f(y))\}= \\
& =\{g(f(x)) \bullet g(f(y)), g(f(y)) \bullet g(f(x))\}
\end{aligned}
$$

Portanto $g \circ f$ é um half-isomorfismo.

Proposição 3.2.14. Sejam $(L, *),\left(L^{\prime}, \cdot\right)$ e $\left(L^{\prime \prime}, \bullet\right)$ loops e $f: L \rightarrow L^{\prime}$ e $g: L^{\prime} \rightarrow L^{\prime \prime}$ halfisomorfismos. Então

a) Se $f$ e $g$ são isomorfismos, então $g \circ f$ é um isomorfismo.

b) Se $f$ e $g$ são anti-isomorfismos, então $g \circ f$ é um isomorfismo.

c) Se $f$ é um isomorfismo e $g$ é um anti-isomorfismo, então $g \circ f$ é um anti-isomorfismo.

d) Se $f$ é um anti-isomorfismo e g é um isomorfismo, então $g \circ f$ é um anti-isomorfismo.

e) Se $f$ é um half-isomorfismo não trivial e $g$ é um half-isomorfismo trivial, então $g \circ f$ é um half-isomorfismo não trivial.

f) Se $f$ é um half-isomorfismo trivial e $g$ é um half-isomorfismo não trivial, então $g \circ f$ é um half-isomorfismo não trivial.

Demonstração. a) Fixados $x, y \in L$, temos:

$$
g \circ f(x * y)=g(f(x) \cdot f(y))=g(f(x)) \bullet g(f(y))
$$

Logo $g \circ f$ é um isomorfismo.

As demonstrações dos itens b), c) e d) são análogas à do item a).

e) Como $f$ é não trivial, existem $x_{1}, x_{2}, y_{1}, y_{2} \in L$ tais que:

$$
\begin{aligned}
& f\left(x_{1} * y_{1}\right)=f\left(x_{1}\right) \cdot f\left(y_{1}\right) \neq f\left(y_{1}\right) \cdot f\left(x_{1}\right) \\
& f\left(x_{2} * y_{2}\right)=f\left(y_{2}\right) \cdot f\left(x_{2}\right) \neq f\left(x_{2}\right) \cdot f\left(y_{2}\right)
\end{aligned}
$$

Como $g$ é um half-isomorfismo trivial, temos dois casos:

(i) Suponha que $g$ é um isomorfismo.

Assim, aplicando $g$ nas equações 3.3, temos:

$$
\begin{aligned}
& g\left(f\left(x_{1} * y_{1}\right)\right)=g\left(f\left(x_{1}\right)\right) \bullet g\left(f\left(y_{1}\right)\right) \neq g\left(f\left(y_{1}\right)\right) \bullet g\left(f\left(x_{1}\right)\right) \\
& g\left(f\left(x_{2} * y_{2}\right)\right)=g\left(f\left(y_{2}\right)\right) \bullet g\left(f\left(x_{2}\right)\right) \neq g\left(f\left(x_{2}\right)\right) \bullet g\left(f\left(y_{2}\right)\right)
\end{aligned}
$$

Logo, $g \circ f$ é um half-isomorfismo não trivial.

(ii) Suponha que $g$ é um anti-isomorfismo.

Assim, aplicando $g$ nas equações 3.3, temos:

$$
\begin{aligned}
& g\left(f\left(x_{1} * y_{1}\right)\right)=g\left(f\left(y_{1}\right)\right) \bullet g\left(f\left(x_{1}\right)\right) \neq g\left(f\left(x_{1}\right)\right) \bullet g\left(f\left(y_{1}\right)\right) \\
& g\left(f\left(x_{2} * y_{2}\right)\right)=g\left(f\left(x_{2}\right)\right) \bullet g\left(f\left(y_{2}\right)\right) \neq g\left(f\left(y_{2}\right)\right) \bullet g\left(f\left(x_{2}\right)\right)
\end{aligned}
$$


Logo, $g \circ f$ é um half-isomorfismo não trivial.

f) A demonstração é análoga à do item e).

\subsection{O grupo de half-automorfismos}

Para um loop $L$, defina o conjunto dos half-automorfismos de $L$, o conjunto dos half-automorfismos triviais de $L$ e o conjunto dos half-automorfismos não triviais de $L$ respectivamente por:

$$
\begin{aligned}
\operatorname{Half}_{(L)} & =\{f: L \rightarrow L ; f \text { é half-automorfismo }\} \\
\operatorname{Half}_{T}(L) & =\{f: L \rightarrow L ; f \text { é half-automorfismo trivial }\} \\
\operatorname{Half}_{N}(L) & =\{f: L \rightarrow L ; f \text { é half-automorfismo não trivial }\}
\end{aligned}
$$

Note que $\operatorname{Half}(L)=\operatorname{Half}_{T}(L) \cup \operatorname{Half}_{N}(L)$, sendo esta união disjunta.

Das proposições 3.2.13 e 3.2.14, observamos que os conjuntos $\operatorname{Half}_{(L)}(L) \operatorname{Half}_{T}(L)$ são fechados para a operação de composição de funções. Além disso, é fácil ver que a função inversa de um automorfismo é também um automorfismo, e que a função inversa de um anti-automorfismo é também um anti-automorfismo. Com isso, temos que $\operatorname{Half}_{T}(L)$ é fechado para inversos, e portanto ele é um grupo, e será chamado grupo dos half-automorfismos triviais de L. Além disso, sendo $f$ um automorfismo e $g$ um anti-automorfismo, da proposição 3.2.14 temos que $g^{-1} f g$ é um automorfismo, e assim $\varphi^{-1} \operatorname{Aut}(L) \varphi$ está contido em $\operatorname{Aut}(L)$, para todo $\varphi$ pertencente a $\operatorname{Half}_{T}(L)$ e, portanto, $\operatorname{Aut}(L) \triangleleft \operatorname{Half}_{T}(L)$. Desta forma, estabelecemos o resultado a seguir.

Proposição 3.3.1. Seja L loop. Então $\operatorname{Half}_{T}(L)$ é um grupo. Além disso, Aut $(L) \triangleleft \operatorname{Half}_{T}(L)$.

Agora para o conjunto Half $(L)$ ser um grupo, precisamos de informações adicionais. Como Half $(L)$ é fechado para a operação de composição de funções, se ele for fechado para inversos, ele será um grupo. Reciprocamente, se $\operatorname{Half}(L)$ é grupo, então ele é fechado para inversos. Com isso, estabelecemos o resultado a seguir.

Proposição 3.3.2. Seja L loop. Então Half $(L)$ é um grupo se, e somente se, todos os halfautomorfismos de L forem especiais.

Observação 3.3.3. Dos corolários 3.2 .8 e 3.2.9, segue que se um loop $L$ é finito ou se $K(L)$ é finito, então $\operatorname{Half}(L)$ é grupo. 


\section{Capítulo 4}

\section{Half-isomorfismos de loops automórficos diedrais}

Neste capítulo introduzimos uma classe de $A$-loops que foi apresentada primeiramente por M.K. Kinyon, K. Kunen, J.D. Phillips e P. Vojtěchovský em [KKPV16]. Os autores construiram uma classe de $A$-loops a partir da duplicação de um grupo abeliano $G$ e de um de seus automorfismos. Aqui chamamos um loop resultante desta construção de loop automórfico diedral. Neste mesmo artigo, os autores provaram que todo $A$-loop não associativo de ordem $2 p$, com $p$ primo ímpar, é um loop automórfico diedral construído a partir de $\mathbb{Z}_{p}$. Provaram ainda que existem $p-2$ destes loops. Essa caracterização torna o estudo desta classe de $A$-loops ainda mais importante. Na dissertação [dA14] e no artigo de M. Aboras e P. Vojtěchovský [AV16] foram estudadas esta classe de $A$-loops e o seu grupo de automorfismos, sendo que alguns dos resultados obtidos são utilizados neste capítulo. Aqui analisamos os half-isomorfismos triviais e não trivias nesta classe de $A$-loops.

Na primeira seção definimos o loop automórfico diedral. Na segunda seção estudamos os halfisomorfismos entre dois loops automórficos diedrais construídos a partir do mesmo grupo. Primeiramente mostramos que todo half-isomorfismo entre estes loops é especial. Em seguida, para o caso não comutativo, mostramos a forma tanto dos half-isomorfismos triviais como dos não triviais, obtendo condições para suas existências e suas quantidades. Na terceira seção obtemos expressões para os grupos dos half-automorfismos e dos half-automorfismos triviais de loops automórficos diedrais.

\subsection{Loop automórfico diedral}

Sejam $G$ grupo abeliano finito e $\alpha$ pertencente a $A u t(G)$. Defina o seguinte conjunto:

$$
\operatorname{Dih}(\alpha, G):=\mathbb{Z}_{2} \times G
$$

Definimos a operação $*$ em $\operatorname{Dih}(\alpha, G)$ da seguinte maneira:

$$
(i, u) *(j, v):=\left(i+j, \alpha^{i j}\left(u^{(-1)^{j}} v\right)\right) \quad i, j \in \mathbb{Z}_{2}, u, v \in G
$$

ou, equivalentemente, para $u$ e $v$ pertencentes a $G$ :

$(0, u) *(0, v)=(0, u v)$

$(0, u) *(1, v)=\left(1, u^{-1} v\right)$

$(1, u) *(0, v)=(1, u v)$

$(1, u) *(1, v)=\left(0, \alpha\left(u^{-1} v\right)\right)$

A partir desta definição, podemos observar que: 
(i) O elemento $(0, e)$ é a identidade de $\operatorname{Dih}(\alpha, G)$, onde $e$ é a identidade de $G$.

(ii) $(0, G)=\{(0, x) \mid x \in G\}$ é subgrupo abeliano de $\operatorname{Dih}(\alpha, G)$ isomorfo a $G$.

(iii) $o((0, u))=o(u)$, para todo $u$ pertencente a $G$.

(iv) Os elementos da forma $(1, u)$ possuem ordem 2.

Além disto, sendo $I_{d}$ a função identidade em $A u t(G)$, temos o seguinte resultado:

Proposição 4.1.1. ([KKPV16] Prop. 9.1) Sejam $G$ grupo abeliano e a pertencente a Aut $(G)$. Então Dih $(\alpha, G)$ é A-loop. Se $\alpha$ é diferente de $I_{d}$, então $N_{\mu}(\operatorname{Dih}(\alpha, G))=(0, G)$.

Os $A$-loops do tipo Dih $(\alpha, G)$ são chamados loops automórficos diedrais. A seguir, dois resultados que caracterizam quando este tipo de $A$-loop é associativo ou comutativo.

Teorema 4.1.2. ([dA14] Teorema 3.1.2) Sejam G grupo abeliano e a pertencente a Aut $(G)$. Então $\operatorname{Dih}(\alpha, G)$ é grupo se, e somente se, $\alpha=I_{d}$. Além disso, se $\alpha$ é diferente de $I_{d}$, temos:

$$
N_{\lambda}(\operatorname{Dih}(\alpha, G))=\{(0, u) \in \operatorname{Dih}(\alpha, G) \mid \alpha(u)=u\}
$$

Teorema 4.1.3. ([dA14] Teorema 3.1.3) Sejam G grupo abeliano e a pertencente a Aut $(G)$. Então $D i h(\alpha, G)$ é comutativo se, e somente se, $G$ tem período 2. Além disso, se o período de $G$ é diferente de 2, temos:

$$
\begin{gathered}
C(\operatorname{Dih}(\alpha, G))=\left\{(0, u) \in \operatorname{Dih}(\alpha, G) \mid u=u^{-1}\right\} \\
\mathcal{Z}(\operatorname{Dih}(\alpha, G))=\left\{(0, u) \in \operatorname{Dih}(\alpha, G) \mid \alpha(u)=u \text { e } u=u^{-1}\right\}
\end{gathered}
$$

onde $C(\operatorname{Dih}(\alpha, G))$ e $\mathcal{Z}(\operatorname{Dih}(\alpha, G))$ são respectivamente o comutante e o centro de Dih $(\alpha, G)$.

\subsection{Half-isomorfismos entre $L_{\alpha}$ e $L_{\beta}$}

Nesta seção, denotaremos por $L_{\alpha}$ o loop automórfico diedral $\operatorname{Dih}(\alpha, G)$. Além disso para $\alpha$ e $\beta$ pertencentes a $\operatorname{Aut}(G)$, denotaremos por $* \mathrm{e} \cdot$ respectivamente as operações de $L_{\alpha}$ e $L_{\beta}$. Nosso objetivo será caracterizar os half-isomorfismos entre $L_{\alpha}$ e $L_{\beta}$.

No capítulo 3, para um loop $L$, definimos o conjunto $K(L)=\{(x, y) \in L \times L \mid x y=y x\}$. A partir da definição da operação em $L_{\alpha}$, para $u$ e $v$ pertencentes a $G$ temos:

$(0, u) *(0, v)=(0, v) *(0, u)$

$(0, u) *(1, v)=(1, v) *(0, u)$ se, e somente se, $u=u^{-1}$

$(1, u) *(1, v)=(1, v) *(1, u)$ se, e somente se, $u^{2}=v^{2}$

Assim:

$K\left(L_{\alpha}\right)=\left\{\left(\left(0, u_{1}\right),\left(0, v_{1}\right)\right),\left(\left(0, u_{2}\right),\left(1, v_{2}\right)\right),\left(\left(1, u_{3}\right),\left(1, v_{3}\right)\right) \mid u_{1}, u_{2}, u_{3}, v_{1}, v_{2}, v_{3} \in G, u_{2}=u_{2}^{-1}, u_{3}^{2}=v_{3}^{2}\right\}$

Logo, é fácil ver que $K\left(L_{\alpha}\right)=K\left(L_{\beta}\right)$. Desta forma, segue do teorema 3.2.7 o resultado a seguir.

Proposição 4.2.1. Seja $f: L_{\alpha} \rightarrow L_{\beta}$ um half-isomorfismo. Então $f$ é especial. 
Desta forma, os loops automórficos diedrais constituem uma classe de loop onde todo halfisomorfismo é especial.

A partir do resultado anterior e das proposições 3.2 .1 e 3.2.10 podemos estabelecer o seguinte resultado:

Proposição 4.2.2. Seja $f: L_{\alpha} \rightarrow L_{\beta}$ um half-isomorfismo. Se $L_{\alpha}$ ou $L_{\beta}$ é comutativo, então $f$ é isomorfismo.

Com este resultado, vemos que, se $L_{\alpha}$ ou $L_{\beta}$ é comutativo, então o estudo de half-isomorfismos se reduz ao estudo de automorfismos de loops automórficos diedrais, o que foi feito em [AV16].

De agora em diante, $L_{\alpha}$ e $L_{\beta}$ serão não comutativos e, do teorema 4.1.3, $G$ será tal que o seu período é diferente de 2 .

Proposição 4.2.3. Seja $f: L_{\alpha} \rightarrow L_{\beta}$ um half-isomorfismo. Então $f((0, G))=(0, G)$.

Demonstração. Como o período de $G$ é diferente de 2, existe $u \in G$ tal que $o(u)>2$.

Pela proposição 3.2.4, segue que $f$ preserva a ordem dos elementos de $L_{\alpha}$. Desta forma, como $o((1, v))=2$, para todo $v \in G$, segue que $f((0, u))=\left(0, u^{\prime}\right)$, com $u^{\prime} \in G$.

Suponha, por absurdo, que existe $v \in G$ tal que $f((0, v))=\left(1, v^{\prime}\right)$, com $v^{\prime} \in G$. Como $f$ preserva a ordem, temos que $o(v)=2$. Desta forma:

$$
(u v)^{2}=u^{2} v^{2}=u^{2} \neq e
$$

Logo $o(u v)>2$ e assim temos $f((0, u v))=(0, w)$, para algum $w \in G$. Com isto:

$$
\begin{aligned}
& (0, w)=f((0, u v))=f((0, u) *(0, v)) \in\{f((0, u)) \cdot f((0, v)), f((0, v)) \cdot f((0, u))\}= \\
& \left\{\left(0, u^{\prime}\right) \cdot\left(1, v^{\prime}\right),\left(1, v^{\prime}\right) \cdot\left(0, u^{\prime}\right)\right\}=\left\{\left(1, u^{\prime-1} v^{\prime}\right),\left(1, v^{\prime} u^{\prime}\right)\right\}
\end{aligned}
$$

Assim, temos um absurdo. Logo $f((0, G)) \subseteq(0, G)$, e como $f$ é bijetora e $G$ finito, segue que $f((0, G))=(0, G)$.

Seja $f: L_{\alpha} \rightarrow L_{\beta}$ um half-isomorfismo. Defina $f^{\prime}: G \rightarrow G$ por:

$$
\left(0, f^{\prime}(u)\right)=f((0, u))
$$

Pela proposição 4.2.3 temos que $f^{\prime}$ está bem definida. Além disso, temos o seguinte resultado:

Proposição 4.2.4. A função $f^{\prime}$ definida anteriormente é um automorfismo de $G$.

Demonstração. Para $u, v \in G$, temos:

$$
\begin{aligned}
&\left(0, f^{\prime}(u v)\right)=f((0, u v))=f((0, u) *(0, v)) \in\{f((0, u)) \cdot f((0, v)), f((0, v)) \cdot f((0, u))\}= \\
&=\left\{\left(0, f^{\prime}(u)\right) \cdot\left(0, f^{\prime}(v)\right),\left(0, f^{\prime}(v)\right) \cdot\left(0, f^{\prime}(u)\right)\right\}=\left\{\left(0, f^{\prime}(u) f^{\prime}(v)\right)\right\}
\end{aligned}
$$

Logo, $f^{\prime}(u v)=f^{\prime}(u) f^{\prime}(v)$. 
Proposição 4.2.5. Seja $f: L_{\alpha} \rightarrow L_{\beta}$ um half-isomorfismo. Então existe a pertencente a $G$ tal que para todo u pertencente a $G$, temos:

$$
f((i, u))=\left\{\begin{array}{ll}
\left(0, f^{\prime}(u)\right), & i=0 \\
\left(1, a f^{\prime}\left(u^{\epsilon_{u}}\right)\right), & i=1
\end{array} \quad\left(\epsilon_{u} \in\{-1,1\}\right)\right.
$$

Demonstração. Seja $a \in G$ tal que $f((1, e))=(1, a)$. Como $f((0, u))=\left(0, f^{\prime}(u)\right)$, temos:

$$
\begin{aligned}
& f((1, u))=f((1, e) *(0, u)) \in\{f((1, e)) \cdot f((0, u)), f((0, u)) \cdot f((1, e))\}= \\
= & \left\{(1, a) \cdot\left(0, f^{\prime}(u)\right),\left(0, f^{\prime}(u)\right) \cdot(1, a)\right\}=\left\{\left(1, a f^{\prime}(u)\right),\left(1, a f^{\prime}\left(u^{-1}\right)\right)\right\}
\end{aligned}
$$

Seja $f^{\prime}: G \rightarrow G$ um automorfismo e $a$ pertencente a $G$. Defina $f_{-a}, f_{+a}: L_{\alpha} \rightarrow L_{\beta}$ por

$$
f_{-a}((i, u))=\left\{\begin{array}{ll}
\left(0, f^{\prime}(u)\right), & i=0 \\
\left(1, a f^{\prime}\left(u^{-1}\right)\right), & i=1
\end{array} \quad f_{+a}((i, u))= \begin{cases}\left(0, f^{\prime}(u)\right) & i=0 \\
\left(1, a f^{\prime}(u)\right) & i=1\end{cases}\right.
$$

Podemos observar que como $G$ não tem período 2 , então $f_{-a}$ é diferente de $f_{+a}$, para todo $a$ pertencente a $G$.

Defina a função $J: G \rightarrow G$ por $J(x)=x^{-1}$. Como $G$ é comutativo, então $J$ é um automorfismo de $G$. Além disso, $J$ pertence a $\mathcal{Z}(A u t(G))$, onde $\mathcal{Z}(A u t(G))$ denota o centro do grupo de automorfismos de $G$.

Proposição 4.2.6. Seja $f^{\prime}: G \rightarrow G$ um automorfismo e a pertencente a $G$. Então:

a) Se $f^{\prime} \alpha=\beta f^{\prime}$, então $f_{+a}$ é isomorfismo e $f_{-a}$ é anti-isomorfismo.

b) Se $f^{\prime} \alpha=\beta f^{\prime} J$, então $f_{+a}$ e $f_{-a}$ são half-isomorfismos não triviais.

Demonstração. Provaremos os resultados para cada função separadamente.

(i) Considere a função $f_{+a}$. Temos quatro casos:

(i.1) Sejam $(0, u),(0, v) \in L_{\alpha}$. Então:

$f_{+a}((0, u) *(0, v))=f_{+a}((0, u v))=\left(0, f^{\prime}(u v)\right)=\left(0, f^{\prime}(u)\right) \cdot\left(0, f^{\prime}(v)\right)=f_{+a}((0, u)) \cdot f_{+a}((0, v))$

(i.2) Sejam $(0, u),(1, v) \in L_{\alpha}$. Então:

$f_{+a}((0, u) *(1, v))=f_{+a}\left(\left(1, u^{-1} v\right)\right)=\left(1, a f^{\prime}\left(u^{-1} v\right)\right)=\left(0, f^{\prime}(u)\right) \cdot\left(1, a f^{\prime}(v)\right)=$

$=f_{+a}((0, u)) \cdot f_{+a}((1, v))$

(i.3) Sejam $(1, u),(0, v) \in L_{\alpha}$. Então:

$$
f_{+a}((1, u) *(0, v))=f_{+a}((1, u v))=\left(1, a f^{\prime}(u v)\right)=\left(1, a f^{\prime}(u)\right) \cdot\left(0, f^{\prime}(v)\right)=f_{+a}((1, u)) \cdot f_{+a}((0, v))
$$

(i.4) Sejam $(1, u),(1, v) \in L_{\alpha}$. Então:

$$
\begin{aligned}
& f_{+a}((1, u) *(1, v))=f_{+a}\left(\left(0, \alpha\left(u^{-1} v\right)\right)\right)=\left(0, f^{\prime} \alpha\left(u^{-1} v\right)\right) \\
& f_{+a}((1, u)) \cdot f_{+a}((1, v))=\left(1, a f^{\prime}(u)\right) \cdot\left(1, a f^{\prime}(v)\right)=\left(0, \beta f^{\prime}\left(u^{-1} v\right)\right) \\
& f_{+a}((1, v)) \cdot f_{+a}((1, u))=\left(1, a f^{\prime}(v)\right) \cdot\left(1, a f^{\prime}(u)\right)=\left(0, \beta f^{\prime} J\left(u^{-1} v\right)\right)
\end{aligned}
$$


Agora temos dois casos, dependendo do comportamento de $f^{\prime} \alpha$.

(i.4.1) Suponha que $f^{\prime} \alpha=\beta f^{\prime}$

Assim $f_{+a}((1, u) *(1, v))=f_{+a}((1, u)) \cdot f_{+a}((1, v))$. Logo, disto juntamente com os casos (i.1), (i.2) e (i.3) segue que $f_{+a}$ é um isomorfismo.

(i.4.2) Suponha que $f^{\prime} \alpha=\beta f^{\prime} J$

Assim $f_{+a}((1, u) *(1, v))=f_{+a}((1, v)) \cdot f_{+a}((1, u))$. Logo, disto juntamente com os casos (i.1), (i.2) e (i.3) segue que $f_{+a}$ é um half-isomorfismo. Agora veremos que ele é não trivial. Como $G$ não tem período 2 , seja $w \in G$ tal que $w \neq w^{-1}$. temos:

De (i.3) temos que $f_{+a}((1, e) *(0, w))=f_{+a}((1, e)) \cdot f_{+a}((0, w))=\left(1, a f^{\prime}(w)\right)$. Por outro lado,

$$
f_{+a}((0, w)) \cdot f_{+a}((1, e))=\left(0, f^{\prime}(w)\right) \cdot(1, a)=\left(1, a f^{\prime}\left(w^{-1}\right)\right) \stackrel{\substack{w \neq w^{-1} \\ \neq}}{\neq}\left(1, a f^{\prime}(w)\right)
$$

Assim temos que $f_{+a}((1, e) *(0, w)) \neq f_{+a}((0, w)) \cdot f_{+a}((1, e))$, o que implica que $f_{+a}$ não é um anti-isomorfismo. lado:

Além disso, vimos que $f_{+a}((1, w) *(1, e))=f_{+a}((1, e)) \cdot f_{+a}((1, w))=\left(0, f^{\prime} \alpha\left(w^{-1}\right)\right)$. Por outro

$$
f_{+a}((1, w)) \cdot f_{+a}((1, e))=\left(0, \beta f^{\prime}\left(w^{-1}\right)\right)=\left(0, \beta f^{\prime} J(w)\right)=\left(0, f^{\prime} \alpha(w)\right) \stackrel{\substack{w \neq w^{-1} \\ \neq}}{\neq}\left(0, f^{\prime} \alpha\left(w^{-1}\right)\right)
$$

Desta forma $f_{+a}((1, w) *(1, e)) \neq f_{+a}((1, w)) \cdot f_{+a}((1, e))$, logo $f_{+a}$ não é um isomorfismo. Portanto $f_{+a}$ é um half-isomorfismo não trivial.

(ii) Considere a função $f_{-a}$.

(ii.1) Sejam $(0, u),(0, v) \in L_{\alpha}$. Então:

$f_{-a}((0, u) *(0, v))=f_{-a}((0, u v))=\left(0, f^{\prime}(u v)\right)=\left(0, f^{\prime}(v)\right) \cdot\left(0, f^{\prime}(u)\right)=f_{-a}((0, v)) \cdot f_{-a}((0, u))$

(ii.2) Sejam $(0, u),(1, v) \in L_{\alpha}$. Então:

$f_{-a}((0, u) *(1, v))=f_{-a}\left(\left(1, u^{-1} v\right)\right)=\left(1, a f^{\prime}\left(u v^{-1}\right)\right)=\left(1, a f^{\prime}\left(v^{-1}\right)\right) \cdot\left(0, f^{\prime}(u)\right)=$

$=f_{-a}((1, v)) \cdot f_{-a}((0, u))$

(ii.3) Sejam $(1, u),(0, v) \in L_{\alpha}$. Então:

$f_{-a}((1, u) *(0, v))=f_{-a}((1, u v))=\left(1, a f^{\prime}\left(u^{-1} v^{-1}\right)\right)=\left(0, f^{\prime}(v)\right) \cdot\left(1, a f^{\prime}\left(u^{-1}\right)\right)=$

$=f_{-a}((0, v)) \cdot f_{-a}((1, u))$

(ii.4) Sejam $(1, u),(1, v) \in L_{\alpha}$. Então:

$$
\begin{aligned}
& f_{-a}((1, u) *(1, v))=f_{-a}\left(\left(0, \alpha\left(u^{-1} v\right)\right)\right)=\left(0, f^{\prime} \alpha\left(u^{-1} v\right)\right) \\
& f_{-a}((1, u)) \cdot f_{-a}((1, v))=\left(1, a f^{\prime}\left(u^{-1}\right)\right) \cdot\left(1, a f^{\prime}\left(v^{-1}\right)\right)=\left(0, \beta f^{\prime} J\left(u^{-1} v\right)\right) \\
& f_{-a}((1, v)) \cdot f_{-a}((1, u))=\left(1, a f^{\prime}\left(v^{-1}\right)\right) \cdot\left(1, a f^{\prime}\left(u^{-1}\right)\right)=\left(0, \beta f^{\prime}\left(u^{-1} v\right)\right)
\end{aligned}
$$


Agora temos dois casos, dependendo do comportamento de $f^{\prime} \alpha$.

(ii.4.1) Suponha que $f^{\prime} \alpha=\beta f^{\prime}$

Assim $f_{-a}((1, u) *(1, v))=f_{-a}((1, v)) \cdot f_{-a}((1, u))$. Logo, disto juntamente com os casos (ii.1), (ii.2) e (ii.3) segue que $f_{-a}$ é um anti-isomorfismo.

(ii.4.2) Suponha que $f^{\prime} \alpha=\beta f^{\prime} J$

Assim $f_{-a}((1, u) *(1, v))=f_{-a}((1, u)) \cdot f_{-a}((1, v))$. Logo, disto juntamente com os casos (ii.1), (ii.2) e (ii.3) segue que $f_{-a}$ é um half-isomorfismo. Agora veremos que ele é não trivial. Como $G$ não tem período 2 , seja $w \in G$ tal que $w \neq w^{-1}$.

De (ii.3) temos que $f_{-a}((1, e) *(0, w))=f_{-a}((0, w)) \cdot f_{-a}((1, e))=\left(1, a f^{\prime}\left(w^{-1}\right)\right)$. Por outro lado temos:

$$
f_{-a}((1, e)) \cdot f_{-a}((0, w))=(1, a) \cdot\left(0, f^{\prime}(w)\right)=\left(1, a f^{\prime}(w)\right) \stackrel{\substack{w \neq w^{-1} \\ \neq}}{\neq}\left(1, a f^{\prime}\left(w^{-1}\right)\right)
$$

Assim temos que $f_{-a}((1, e) *(0, w)) \neq f_{-a}((1, e)) \cdot f_{-a}((0, w))$, o que implica que $f_{-a}$ não é um isomorfismo. lado:

Além disso, vimos que $f_{-a}((1, w) *(1, e))=f_{-a}((1, w)) \cdot f_{-a}((1, e))=\left(0, f^{\prime} \alpha\left(w^{-1}\right)\right)$. Por outro

$$
f_{-a}((1, e)) \cdot f_{-a}((1, w))=\left(0, \beta f^{\prime}\left(w^{-1}\right)\right)=\left(0, \beta f^{\prime} J(w)\right)=\left(0, f^{\prime} \alpha(w)\right) \stackrel{w \neq w^{-1}}{\neq}\left(0, f^{\prime} \alpha\left(w^{-1}\right)\right)
$$

Desta forma $f_{-a}((1, w) *(1, e)) \neq f_{-a}((1, e)) \cdot f_{-a}((1, w))$, logo $f_{-a}$ não é um anti-isomorfismo. Portanto $f_{-a}$ é um half-isomorfismo não trivial.

Lema 4.2.7. Sejam $L$ um loop e $H, K$ subloops de $L$ tais que $L=H \cup K$. Então $L$ pertence a $\{H, K\}$.

Demonstração. Se $H$ é subloop de $K$ ou se $K$ é subloop de $H$, temos que $L=H \cup K \in\{H, K\}$. Suponha que $H$ não é subloop de $K$ e que $K$ não é subloop de $H$. Assim, existem $x, y \in L$ tais que $x \in H-K$ e $y \in K-H$.

Como $x y \in L=H \cup K$, ou $x y \in H$, ou $x y \in K$. Se $x y \in H$, como $x \in H$, segue que $y \in H$. Mas $y \in K-H$, e assim temos um absurdo. Analogamente, se $x y \in K$ teremos um absurdo.

Proposição 4.2.8. Seja $f: L_{\alpha} \rightarrow L_{\beta}$ um half-isomorfismo. Então $f^{\prime} \alpha$ pertence a $\left\{\beta f^{\prime}, \beta f^{\prime} J\right\}$.

Demonstração. Das proposições 4.2.4 e 4.2.5 segue que $f^{\prime}: G \rightarrow G$ é automorfismo e existe $a \in G$ tal que para todo $u \in G$ :

$$
f((i, u))=\left\{\begin{array}{ll}
\left(0, f^{\prime}(u)\right), & i=0 \\
\left(1, a f^{\prime}\left(u^{\epsilon_{u}}\right)\right), & i=1
\end{array} \quad\left(\epsilon_{u} \in\{-1,1\}\right)\right.
$$

Assim, como $f((1, e))=(1, a)$, temos que para todo $v \in G$ :

$$
\begin{aligned}
& f((1, e) *(1, v))=f((0, \alpha(v)))=\left(0, f^{\prime} \alpha(v)\right) \\
& f((1, e)) \cdot f((1, v))=(1, a) \cdot\left(1, a f^{\prime}\left(v^{\epsilon_{v}}\right)\right)=\left(0, \beta f^{\prime}\left(v^{\epsilon_{v}}\right)\right)
\end{aligned}
$$


$f((1, v)) \cdot f((1, e))=\left(1, a f^{\prime}\left(v^{\epsilon_{v}}\right)\right) \cdot(1, a)=\left(0, \beta f^{\prime}\left(v^{-\epsilon_{v}}\right)\right)$

Como $f$ é half-isomorfismo, segue que para todo $v \in G$ :

$f^{\prime} \alpha(v) \in\left\{\beta f^{\prime}\left(v^{\epsilon_{v}}\right), \beta f^{\prime}\left(v^{-\epsilon_{v}}\right)\right\}=\left\{\beta f^{\prime}(v), \beta f^{\prime}\left(v^{-1}\right)\right\}$

Defina:

$H=\left\{v \in G \mid f^{\prime} \alpha(v)=\beta f^{\prime}(v)\right\}$

$K=\left\{v \in G \mid f^{\prime} \alpha(v)=\beta f^{\prime} J(v)\right\}$

De $(*)$ segue que $G=H \cup K$. Como $\beta f^{\prime}(e)=\beta f^{\prime} J(e)=e=f^{\prime} \alpha(e)$, segue que $e \in H \cap K$.

(i) Vamos mostrar que $H$ é subgrupo de $G$

Para $x, y \in H$, temos que $f^{\prime} \alpha(x)=\beta f^{\prime}(x)$ e $f^{\prime} \alpha(y)=\beta f^{\prime}(y)$. Logo:

$$
f^{\prime} \alpha(x y)=f^{\prime} \alpha(x) f^{\prime} \alpha(y)=\beta f^{\prime}(x) \beta f^{\prime}(y)=\beta f^{\prime}(x y)
$$

Assim, $x y \in H$. Além disso:

$$
e=f^{\prime} \alpha(e)=f^{\prime} \alpha\left(x x^{-1}\right)=f^{\prime} \alpha(x) f^{\prime} \alpha\left(x^{-1}\right)=\beta f^{\prime}(x) f^{\prime} \alpha\left(x^{-1}\right)
$$

Desta forma, $f^{\prime} \alpha\left(x^{-1}\right)=\beta f^{\prime}\left(x^{-1}\right)$, e temos que $x^{-1} \in H$. Portanto $H$ é subgrupo de $G$.

(ii) Vamos mostrar que $K$ é subgrupo de $G$

Para $x, y \in H$, temos que $f^{\prime} \alpha(x)=\beta f^{\prime} J(x)$ e $f^{\prime} \alpha(y)=\beta f^{\prime} J(y)$. Logo:

$$
f^{\prime} \alpha(x y)=f^{\prime} \alpha(x) f^{\prime} \alpha(y)=\beta f^{\prime} J(x) \beta f^{\prime} J(y)=\beta f^{\prime} J(x y)
$$

Assim, $x y \in K$. Além disso:

$$
e=f^{\prime} \alpha(e)=f^{\prime} \alpha\left(x x^{-1}\right)=f^{\prime} \alpha(x) f^{\prime} \alpha\left(x^{-1}\right)=\beta f^{\prime} J(x) f^{\prime} \alpha\left(x^{-1}\right)
$$

Desta forma, $f^{\prime} \alpha\left(x^{-1}\right)=\beta f^{\prime} J\left(x^{-1}\right)$, e temos que $x^{-1} \in K$. Portanto $K$ é subgrupo de $G$.

Logo, como $G$ é a união de dois subgrupos, do lema 4.2 .7 segue que $G \in\{H, K\}$. Assim $f^{\prime} \alpha \in\left\{\beta f^{\prime}, \beta f^{\prime} J\right\}$.

Proposição 4.2.9. Seja $f: L_{\alpha} \rightarrow L_{\beta}$ um half-isomorfismo. Se $f^{\prime} \alpha$ pertence a $\left\{\beta f^{\prime}, \beta f^{\prime} J\right\}$, então $f$ pertence $a\left\{f_{-a}, f_{+a} \mid a \in G\right\}$.

Demonstração. Segue da proposição 4.2 .5 que existe $a \in G$ tal que para todo $x \in G$ :

$$
f((i, x))=\left\{\begin{array}{ll}
\left(0, f^{\prime}(x)\right), & i=0 \\
\left(1, a f^{\prime}\left(x^{\epsilon_{x}}\right)\right), & i=1
\end{array} \quad\left(\epsilon_{x} \in\{-1,1\}\right)\right.
$$

(i) Caso 1: $f^{\prime} \alpha=\beta f^{\prime}$.

Seja $u \in G$ tal que $u \neq u^{-1}$. Como $f((1, u))=\left(1, a f^{\prime}\left(u^{ \pm 1}\right)\right)$, temos dois casos:

(i.1) Suponha que $f((1, u))=\left(1, a f^{\prime}(u)\right)$.

Queremos mostrar que $f=f_{+a}$. Sabemos que $f((0, x))=\left(0, f^{\prime}(x)\right)=f_{+a}((0, x))$, para todo $x \in G$. Assim basta mostrarmos que: 


$$
f((1, v))=\left(1, a f^{\prime}(v)\right), \text { para todo } v \in G \backslash_{\{u\}}
$$

Temos mais dois casos:

(i.1.1) Suponha que $v=v^{-1}$.

$\operatorname{Logo} f((1, v))=\left(1, a f^{\prime}\left(v^{ \pm 1}\right)\right)=\left(1, a f^{\prime}(v)\right)$.

(i.1.2) Suponha que $v \neq v^{-1}$.

Como $u$ e $v$ são diferentes dos seus inversos, segue que:

$$
u^{-1} v \notin\left\{u v, u^{-1} v^{-1}\right\}
$$

Temos que $f((1, v))=\left(1, a f^{\prime}\left(v^{\epsilon_{x}}\right)\right)$, com $\epsilon_{v} \in\{-1,1\}$. Queremos mostrar que $\epsilon_{v}=1$.

$$
\begin{aligned}
& f((1, u) *(1, v))=f\left(\left(0, \alpha\left(u^{-1} v\right)\right)\right)=\left(0, f^{\prime} \alpha\left(u^{-1} v\right)\right) \stackrel{f^{\prime} \alpha=\beta f^{\prime}}{=}\left(0, \beta f^{\prime}\left(u^{-1} v\right)\right) \\
& f((1, u)) \cdot f((1, v))=\left(1, a f^{\prime}(u)\right) \cdot\left(1, a f^{\prime}\left(v^{\epsilon_{v}}\right)\right)=\left(0, \beta f^{\prime}\left(u^{-1} v^{\epsilon_{v}}\right)\right) \\
& f((1, v)) \cdot f((1, u))=\left(1, a f^{\prime}\left(v^{\epsilon_{v}}\right)\right) \cdot\left(1, a f^{\prime}(u)\right)=\left(0, \beta f^{\prime}\left(u v^{-\epsilon_{v}}\right)\right)
\end{aligned}
$$

Como $f$ é half-isomorfismo, temos:

$$
\begin{aligned}
& f((1, u) *(1, v)) \in\{f((1, u)) \cdot f((1, v)), f((1, v)) \cdot f((1, u))\} \\
& =>\left(0, \beta f^{\prime}\left(u^{-1} v\right)\right) \in\left\{\left(0, \beta f^{\prime}\left(u^{-1} v^{\epsilon_{v}}\right)\right),\left(0, \beta f^{\prime}\left(u v^{-\epsilon_{v}}\right)\right)\right\} \\
& =>\beta f^{\prime}\left(u^{-1} v\right) \in\left\{\beta f^{\prime}\left(u^{-1} v^{\epsilon_{v}}\right), \beta f^{\prime}\left(u v^{-\epsilon_{v}}\right)\right\} \\
& \beta f^{\prime} \text { é bijeção } \\
& \stackrel{=}{-1} v \in\left\{u^{-1} v^{\epsilon_{v}}, u v^{-\epsilon_{v}}\right\}
\end{aligned}
$$

Note que se $\epsilon_{v}=-1$, então $u^{-1} v \in\left\{u v, u^{-1} v^{-1}\right\}$, o que contraria a equação 4.1. Logo $\epsilon_{v}=1$, e $\operatorname{assim} f((1, v))=\left(1, a f^{\prime}(v)\right)$.

Portanto $f=f_{+a}$.

(i.2) Suponha que $f((1, u))=\left(1, a f^{\prime}\left(u^{-1}\right)\right)$.

Queremos mostrar que $f=f_{-a}$. Sabemos que $f((0, x))=\left(0, f^{\prime}(x)\right)=f_{-a}((0, x))$, para todo $x \in G$. Assim basta mostrarmos que:

$$
f((1, v))=\left(1, a f^{\prime}\left(v^{-1}\right)\right), \text { para todo } v \in G \backslash\{u\}
$$

Temos mais dois casos:

(i.2.1) Suponha que $v=v^{-1}$.

$\operatorname{Logo} f((1, v))=\left(1, a f^{\prime}\left(v^{ \pm 1}\right)\right)=\left(1, a f^{\prime}\left(v^{-1}\right)\right)$.

(i.2.2) Suponha que $v \neq v^{-1}$.

Como $u$ e $v$ são diferentes dos seus inversos, repare que a equação 4.1 continua válida. 
Temos que $f((1, v))=\left(1, a f^{\prime}\left(v^{\epsilon_{x}}\right)\right)$, com $\epsilon_{v} \in\{-1,1\}$. Queremos mostrar que $\epsilon_{v}=-1$.

$$
\begin{aligned}
& f((1, u) *(1, v))=f\left(\left(0, \alpha\left(u^{-1} v\right)\right)\right)=\left(0, f^{\prime} \alpha\left(u^{-1} v\right)\right) \stackrel{f^{\prime} \alpha=\beta f^{\prime}}{=}\left(0, \beta f^{\prime}\left(u^{-1} v\right)\right) \\
& f((1, u)) \cdot f((1, v))=\left(1, a f^{\prime}\left(u^{-1}\right)\right) \cdot\left(1, a f^{\prime}\left(v^{\epsilon_{v}}\right)\right)=\left(0, \beta f^{\prime}\left(u v^{\epsilon_{v}}\right)\right) \\
& f((1, v)) \cdot f((1, u))=\left(1, a f^{\prime}\left(v^{\epsilon_{v}}\right)\right) \cdot\left(1, a f^{\prime}\left(u^{-1}\right)\right)=\left(0, \beta f^{\prime}\left(u^{-1} v^{-\epsilon_{v}}\right)\right)
\end{aligned}
$$

Como $f$ é half-isomorfismo, temos:

$$
\begin{aligned}
& f((1, u) *(1, v)) \in\{f((1, u)) \cdot f((1, v)), f((1, v)) \cdot f((1, u))\} \\
& =>\left(0, \beta f^{\prime}\left(u^{-1} v\right)\right) \in\left\{\left(0, \beta f^{\prime}\left(u v^{\epsilon_{v}}\right)\right),\left(0, \beta f^{\prime}\left(u^{-1} v^{-\epsilon_{v}}\right)\right)\right\} \\
& =>\beta f^{\prime}\left(u^{-1} v\right) \in\left\{\beta f^{\prime}\left(u v^{\epsilon_{v}}\right), \beta f^{\prime}\left(u^{-1} v^{-\epsilon_{v}}\right)\right\} \\
& \beta f^{\prime} \text { é bijeção } \\
& =>
\end{aligned}
$$

Note que se $\epsilon_{v}=1$, então $u^{-1} v \in\left\{u v, u^{-1} v^{-1}\right\}$, o que contraria a equação 4.1. Logo $\epsilon_{v}=-1$, e $\operatorname{assim} f((1, v))=\left(1, a f^{\prime}\left(v^{-1}\right)\right)$.

$$
\text { Portanto } f=f_{-a} \text {. }
$$

(ii) Caso 2: $f^{\prime} \alpha=\beta f^{\prime} J$.

Seja $u \in G$ tal que $u \neq u^{-1}$. Como $f((1, u))=\left(1, a f^{\prime}\left(u^{ \pm 1}\right)\right)$, temos dois casos:

(ii.1) Suponha que $f((1, u))=\left(1, a f^{\prime}(u)\right)$.

Queremos mostrar que $f=f_{+a}$. Sabemos que $f((0, x))=\left(0, f^{\prime}(x)\right)=f_{+a}((0, x))$, para todo $x \in G$. Assim basta mostrarmos que:

$$
f((1, v))=\left(1, a f^{\prime}(v)\right), \text { para todo } v \in G \backslash_{\{u\}}
$$

Temos mais dois casos:

(ii.1.1) Suponha que $v=v^{-1}$.

Logo $f((1, v))=\left(1, a f^{\prime}\left(v^{ \pm 1}\right)\right)=\left(1, a f^{\prime}(v)\right)$.

(ii.1.2) Suponha que $v \neq v^{-1}$.

Como $u$ e $v$ são diferentes dos seus inversos, segue que:

$$
u v^{-1} \notin\left\{u v, u^{-1} v^{-1}\right\}
$$

Temos que $f((1, v))=\left(1, a f^{\prime}\left(v^{\epsilon_{x}}\right)\right)$, com $\epsilon_{v} \in\{-1,1\}$. Queremos mostrar que $\epsilon_{v}=1$.

$$
\begin{aligned}
& f((1, u) *(1, v))=f\left(\left(0, \alpha\left(u^{-1} v\right)\right)\right)=\left(0, f^{\prime} \alpha\left(u^{-1} v\right)\right) \stackrel{f^{\prime} \alpha=\beta f^{\prime} J}{=}\left(0, \beta f^{\prime}\left(u v^{-1}\right)\right) \\
& f((1, u)) \cdot f((1, v))=\left(1, a f^{\prime}(u)\right) \cdot\left(1, a f^{\prime}\left(v^{\epsilon_{v}}\right)\right)=\left(0, \beta f^{\prime}\left(u^{-1} v^{\epsilon_{v}}\right)\right) \\
& f((1, v)) \cdot f((1, u))=\left(1, a f^{\prime}\left(v^{\epsilon_{v}}\right)\right) \cdot\left(1, a f^{\prime}(u)\right)=\left(0, \beta f^{\prime}\left(u v^{-\epsilon_{v}}\right)\right)
\end{aligned}
$$

Como $f$ é half-isomorfismo, temos: 
$f((1, u) *(1, v)) \in\{f((1, u)) \cdot f((1, v)), f((1, v)) \cdot f((1, u))\}$

$=>\left(0, \beta f^{\prime}\left(u v^{-1}\right)\right) \in\left\{\left(0, \beta f^{\prime}\left(u^{-1} v^{\epsilon_{v}}\right)\right),\left(0, \beta f^{\prime}\left(u v^{-\epsilon_{v}}\right)\right)\right\}$

$=>\beta f^{\prime}\left(u v^{-1}\right) \in\left\{\beta f^{\prime}\left(u^{-1} v^{\epsilon_{v}}\right), \beta f^{\prime}\left(u v^{-\epsilon_{v}}\right)\right\}$

$\beta f^{\prime} \stackrel{\text { é bijeção }}{=} u v^{-1} \in\left\{u^{-1} v^{\epsilon_{v}}, u v^{-\epsilon_{v}}\right\}$

Note que se $\epsilon_{v}=-1$, então $u v^{-1} \in\left\{u v, u^{-1} v^{-1}\right\}$, o que contraria a equação 4.2. Logo $\epsilon_{v}=1$, e $\operatorname{assim} f((1, v))=\left(1, a f^{\prime}(v)\right)$.

Portanto $f=f_{+a}$.

(ii.2) Suponha que $f((1, u))=\left(1, a f^{\prime}\left(u^{-1}\right)\right)$.

Queremos mostrar que $f=f_{-a}$. Sabemos que $f((0, x))=\left(0, f^{\prime}(x)\right)=f_{-a}((0, x))$, para todo $x \in G$. Assim basta mostrarmos que:

$$
f((1, v))=\left(1, a f^{\prime}\left(v^{-1}\right)\right), \text { para todo } v \in G \backslash_{\{u\}}
$$

Temos mais dois casos:

(ii.2.1) Suponha que $v=v^{-1}$.

$\operatorname{Logo} f((1, v))=\left(1, a f^{\prime}\left(v^{ \pm 1}\right)\right)=\left(1, a f^{\prime}\left(v^{-1}\right)\right)$.

(ii.2.2) Suponha que $v \neq v^{-1}$.

Como $u$ e $v$ são diferentes dos seus inversos, repare que a equação 4.2 continua válida.

Temos que $f((1, v))=\left(1, a f^{\prime}\left(v^{\epsilon_{x}}\right)\right)$, com $\epsilon_{v} \in\{-1,1\}$. Queremos mostrar que $\epsilon_{v}=-1$.

$f((1, u) *(1, v))=f\left(\left(0, \alpha\left(u^{-1} v\right)\right)\right)=\left(0, f^{\prime} \alpha\left(u^{-1} v\right)\right) \stackrel{f^{\prime} \alpha=\beta f^{\prime} J}{=}\left(0, \beta f^{\prime}\left(u v^{-1}\right)\right)$

$f((1, u)) \cdot f((1, v))=\left(1, a f^{\prime}\left(u^{-1}\right)\right) \cdot\left(1, a f^{\prime}\left(v^{\epsilon_{v}}\right)\right)=\left(0, \beta f^{\prime}\left(u v^{\epsilon_{v}}\right)\right)$

$f((1, v)) \cdot f((1, u))=\left(1, a f^{\prime}\left(v^{\epsilon_{v}}\right)\right) \cdot\left(1, a f^{\prime}\left(u^{-1}\right)\right)=\left(0, \beta f^{\prime}\left(u^{-1} v^{-\epsilon_{v}}\right)\right)$

Como $f$ é half-isomorfismo, temos:

$f((1, u) *(1, v)) \in\{f((1, u)) \cdot f((1, v)), f((1, v)) \cdot f((1, u))\}$

$=>\left(0, \beta f^{\prime}\left(u v^{-1}\right)\right) \in\left\{\left(0, \beta f^{\prime}\left(u v^{\epsilon_{v}}\right)\right),\left(0, \beta f^{\prime}\left(u^{-1} v^{-\epsilon_{v}}\right)\right)\right\}$

$=>\beta f^{\prime}\left(u v^{-1}\right) \in\left\{\beta f^{\prime}\left(u v^{\epsilon_{v}}\right), \beta f^{\prime}\left(u^{-1} v^{-\epsilon_{v}}\right)\right\}$

$\beta f^{\prime} \stackrel{\text { é bijeção }}{=} u v^{-1} \in\left\{u v^{\epsilon_{v}}, u^{-1} v^{-\epsilon_{v}}\right\}$

Note que se $\epsilon_{v}=1$, então $u v^{-1} \in\left\{u v, u^{-1} v^{-1}\right\}$, o que contraria a equação 4.2. Logo $\epsilon_{v}=-1$, e $\operatorname{assim} f((1, v))=\left(1, a f^{\prime}\left(v^{-1}\right)\right)$.

Portanto $f=f_{-a}$.

O próximo teorema nos dá uma caracterização dos half-isomorfismos não triviais entre $L_{\alpha}$ e $L_{\beta}$. 
Teorema 4.2.10. São equivalentes:

a) $f: L_{\alpha} \rightarrow L_{\beta}$ é um half-isomorfismo não trivial.

b) $f$ pertence $a\left\{f_{-a}, f_{+a} \mid a \in G\right\}$, com $f^{\prime}: G \rightarrow G$ automorfismo e $f^{\prime} \alpha=\beta f^{\prime} J$.

Demonstração. (b) => (a) Segue da proposição 4.2.6.

(a) $=>$ (b) Pela proposição 4.2.8 temos que $f^{\prime} \alpha \in\left\{\beta f^{\prime}, \beta f^{\prime} J\right\}$. Logo, da proposição 4.2.9 segue que $f \in\left\{f_{-a}, f_{+a} \mid a \in G\right\}$. Desta forma, por $f$ ser um half-isomorfismo não trivial, da proposição 4.2.6 temos que $f^{\prime} \alpha=\beta f^{\prime} J$.

Para $\phi$ pertencente a $A u t(G)$, o centralizador e a classe de conjugação de $\phi$ em $A u t(G)$ são definidos respectivamente por:

$C_{\text {Aut }(G)}(\phi)=\{\psi \in \operatorname{Aut}(G) \mid \phi \psi=\psi \phi\}$

$C l(\phi)=\left\{\psi \phi \psi^{-1} \mid \psi \in \operatorname{Aut}(G)\right\}$

Corolário 4.2.11. Existe um half-isomorfismo não trivial entre $L_{\alpha}$ e $L_{\beta}$ se, e somente se, $\beta J$ pertence a $C l(\alpha)$.

Demonstração. (=>) Do Teorema 4.2.10 b), existe $f^{\prime}: G \rightarrow G$ automorfismo tal que $f^{\prime} \alpha=\beta f^{\prime} J=\beta J f^{\prime} . \operatorname{Logo} \beta J=f^{\prime} \alpha f^{\prime-1} \in C l(\alpha)$.

$(<=)$ Por hipótese, existe $f^{\prime} \in A u t(G)$ tal que $\beta J=f^{\prime} \alpha f^{\prime-1}$, $\log 0 f^{\prime} \alpha=\beta f^{\prime} J$. Assim pelo Teorema 4.2.10, segue que para todo $f \in\left\{f_{-a}, f_{+a} \mid a \in G\right\}, f: L_{\alpha} \rightarrow L_{\beta}$ é um half-isomorfismo não trivial.

Sejam $L$ e $L^{\prime}$ loops. Seguindo as definições da seção 3.3, vamos definir os conjuntos abaixo:

$\operatorname{Half}\left(L, L^{\prime}\right) \quad:=\left\{f: L \rightarrow L^{\prime} \mid f\right.$ é half-isomorfismo $\}$

$\operatorname{Half}_{T}\left(L, L^{\prime}\right):=\left\{f: L \rightarrow L^{\prime} \mid f\right.$ é half-isomorfismo trivial $\}$

$\operatorname{Half}_{N}\left(L, L^{\prime}\right):=\left\{f: L \rightarrow L^{\prime} \mid f\right.$ é half-isomorfismo não trivial $\}$

Note que $\operatorname{Half}\left(L, L^{\prime}\right)=\operatorname{Half}_{T}\left(L, L^{\prime}\right) \cup \operatorname{Half}_{N}\left(L, L^{\prime}\right)$, onde a união é disjunta.

Corolário 4.2.12. Se $\operatorname{Half}_{N}\left(L_{\alpha}, L_{\beta}\right)$ é não vazio, então:

$$
\left|\operatorname{Half}_{N}\left(L_{\alpha}, L_{\beta}\right)\right|=2|G|\left|C_{A u t(G)}(\alpha)\right|
$$

Demonstração. Do Teorema 4.2.10 segue que:

$\left|\operatorname{Half}_{N}\left(L_{\alpha}, L_{\beta}\right)\right|=2 .|G| \cdot \#\left\{f^{\prime}: G \rightarrow G \mid f^{\prime} \alpha=\beta f^{\prime} J\right\}=$

$=2 .|G| . \#\left\{f^{\prime} \in \operatorname{Aut}(G) \mid \beta J=f^{\prime} \alpha f^{\prime-1}\right\} \stackrel{\beta J \in C l(\alpha)}{=} 2 .|G| \cdot\left|C_{\text {Aut }(G)}(\alpha)\right|$

Observação 4.2.13. Na demonstração do corolário 4.2.12 podemos proceder de maneira análoga com a função $\beta J$ no lugar de $\alpha J$. Com isso, obtemos facilmente que $\left|\operatorname{Half}_{N}\left(L_{\alpha}, L_{\beta}\right)\right|=2|G|\left|C_{A u t(G)}(\beta)\right|$ e também que $\left|C_{A u t(G)}(\alpha)\right|=\left|C_{A u t(G)}(\beta)\right|$. 
Sabemos que se $\alpha=I_{d}$, onde $I_{d}$ é a função identidade de $A u t(G)$, então $L_{I_{d}}$ é o grupo diedral generalizado $\left(D_{2|G|}(G)\right)$. E se $\beta=J$, então $L_{J}$ é um loop automórfico não associativo. Como $J^{2}=I_{d}$, temos o seguinte resultado:

Corolário 4.2.14. Existem half-isomorfismos não triviais entre $L_{I_{d}}$ e $L_{J}$. Além disso:

$$
\left|\operatorname{Half}_{N}\left(L_{I_{d}}, L_{J}\right)\right|=2|G||A u t(G)|
$$

A partir deste corolário, podemos construir infinitos exemplos de half-isomorfismos não triviais entre grupos e loops automórficos não associativos.

Exemplo 4.2.15. Considere $G=C_{3}=\{1,2,3\}$, o grupo cíclio de ordem 3. Assim $L_{I_{d}}$ é o grupo diedral de ordem 6 e $L_{J}$ é o menor loop automórfico não associativo. Abaixo nós temos respectivamente as tabelas de Cayley de $L_{I_{d}}$ e $L_{J}$ :

\begin{tabular}{|c|c|c|c|c|c|c|}
\hline$*$ & $(0,1)$ & $(0,2)$ & $(0,3)$ & $(1,1)$ & $(1,2)$ & $(1,3)$ \\
\hline$(0,1)$ & $(0,1)$ & $(0,2)$ & $(0,3)$ & $(1,1)$ & $(1,2)$ & $(1,3)$ \\
\hline$(0,2)$ & $(0,2)$ & $(0,3)$ & $(0,1)$ & $(1,3)$ & $(1,1)$ & $(1,2)$ \\
\hline$(0,3)$ & $(0,3)$ & $(0,1)$ & $(0,2)$ & $(1,2)$ & $(1,3)$ & $(1,1)$ \\
\hline$(1,1)$ & $(1,1)$ & $(1,2)$ & $(1,3)$ & $(0,1)$ & $(0,2)$ & $(0,3)$ \\
\hline$(1,2)$ & $(1,2)$ & $(1,3)$ & $(1,1)$ & $(0,3)$ & $(0,1)$ & $(0,2)$ \\
\hline$(1,3)$ & $(1,3)$ & $(1,1)$ & $(1,2)$ & $(0,2)$ & $(0,3)$ & $(0,1)$ \\
\hline
\end{tabular}

\begin{tabular}{|c|c|c|c|c|c|c|}
\hline$\cdot$ & $(0,1)$ & $(0,2)$ & $(0,3)$ & $(1,1)$ & $(1,2)$ & $(1,3)$ \\
\hline$(0,1)$ & $(0,1)$ & $(0,2)$ & $(0,3)$ & $(1,1)$ & $(1,2)$ & $(1,3)$ \\
\hline$(0,2)$ & $(0,2)$ & $(0,3)$ & $(0,1)$ & $(1,3)$ & $(1,1)$ & $(1,2)$ \\
\hline$(0,3)$ & $(0,3)$ & $(0,1)$ & $(0,2)$ & $(1,2)$ & $(1,3)$ & $(1,1)$ \\
\hline$(1,1)$ & $(1,1)$ & $(1,2)$ & $(1,3)$ & $(0,1)$ & $(0,3)$ & $(0,2)$ \\
\hline$(1,2)$ & $(1,2)$ & $(1,3)$ & $(1,1)$ & $(0,2)$ & $(0,1)$ & $(0,3)$ \\
\hline$(1,3)$ & $(1,3)$ & $(1,1)$ & $(1,2)$ & $(0,3)$ & $(0,2)$ & $(0,1)$ \\
\hline
\end{tabular}

O grupo $G$ só tem dois automorfismos, as funções $I_{d}$ e $J$. Assim, as expressões para os halfisomorfismos não trivias entre $L_{I_{d}}$ e $L_{J}$ escritos em forma de permutação são:

$$
\begin{array}{ll}
I_{d-1}=((1,2)(1,3)) & I_{d+1}=I \\
I_{d-2}=((1,1)(1,2)) & I_{d+2}=((1,1)(1,2)(1,3)) \\
I_{d-3}=((1,1)(1,3)) & I_{d+3}=((1,1)(1,3)(1,2)) \\
J_{-1}=((0,2)(0,3)) & J_{+1}=((0,2)(0,3))((1,2)(1,3)) \\
J_{-2}=((0,2)(0,3))((1,1)(1,2)(1,3)) & J_{+2}=((0,2)(0,3))((1,1)(1,2)) \\
J_{-3}=((0,2)(0,3))((1,1)(1,3)(1,2)) & J_{+3}=((0,2)(0,3))((1,1)(1,3))
\end{array}
$$

onde $I: L_{I_{d}} \longrightarrow L_{J}$ é dada por $I(x)=x$.

Agora analisaremos os half-isomorfismos triviais entre $L_{\alpha}$ e $L_{\beta}$ (lembrando que um half-isomorfismo é trivial se é um isomorfismo ou um anti-isomorfismo). O próximo teorema nos dá uma caracterização destes. 
Teorema 4.2.16. São equivalentes:

a) $f: L_{\alpha} \rightarrow L_{\beta}$ é um half-isomorfismo trivial.

b) $f$ pertence $a\left\{f_{-a}, f_{+a} \mid a \in G\right\}$, com $f^{\prime}: G \rightarrow G$ automorfismo e $f^{\prime} \alpha=\beta f^{\prime}$.

Demonstração. (b) => (a) Segue da proposição 4.2.6.

(a) $=>$ (b) Pela proposição 4.2.8 temos que $f^{\prime} \alpha \in\left\{\beta f^{\prime}, \beta f^{\prime} J\right\}$. Logo, da proposição 4.2.9 segue que $f \in\left\{f_{-a}, f_{+a} \mid a \in G\right\}$. Desta forma, por $f$ ser um half-isomorfismo trivial, da proposição 4.2.6 temos que $f^{\prime} \alpha=\beta f^{\prime}$.

Corolário 4.2.17. São equivalentes:

a) Existe um half-isomorfismo trivial entre $L_{\alpha}$ e $L_{\beta}$.

b) $\beta$ pertence a $C l(\alpha)$.

c) $L_{\alpha}$ é isomorfo a $L_{\beta}$.

Demonstração. $c)=>a)$ Direto.

$a)=>b$ ) Do Teorema 4.2.16 b), existe $f^{\prime}: G \rightarrow G$ automorfismo tal que $f^{\prime} \alpha=\beta f^{\prime}$. Logo $\beta=f^{\prime} \alpha f^{\prime-1} \in C l(\alpha)$.

$b)=>c$ ) Por hipótese, existe $f^{\prime} \in \operatorname{Aut}(G)$ tal que $\beta=f^{\prime} \alpha f^{\prime-1}$, $\operatorname{logo} f^{\prime} \alpha=\beta f^{\prime}$. Assim pela proposição 4.2.6 segue que $f_{+a}: L_{\alpha} \rightarrow L_{\beta}$ é um isomorfismo para todo $a \in G$.

Corolário 4.2.18. Se $\operatorname{Half}_{T}\left(L_{\alpha}, L_{\beta}\right)$ é não vazio, então:

$$
\left|\operatorname{Half}_{T}\left(L_{\alpha}, L_{\beta}\right)\right|=2|G|\left|C_{A u t(G)}(\alpha)\right|
$$

Demonstração. Do Teorema 4.2.16 segue que:

$$
\begin{aligned}
& \left|\operatorname{Half}_{T}\left(L_{\alpha}, L_{\beta}\right)\right|=2 .|G| \cdot \#\left\{f^{\prime}: G \rightarrow G \mid f^{\prime} \alpha=\beta f^{\prime}\right\}= \\
& =2 .|G| . \#\left\{f^{\prime} \in \operatorname{Aut}(G) \mid \beta=f^{\prime} \alpha f^{\prime-1}\right\} \stackrel{\beta \in C l(\alpha)}{=} 2 .|G| \cdot\left|C_{\text {Aut }(G)}(\alpha)\right|
\end{aligned}
$$

Observação 4.2.19. Analogamente à observação 4.2.13, é fácil ver que nas condições do corolário 4.2.18 também podemos obter que $\left|\operatorname{Half}_{N}\left(L_{\alpha}, L_{\beta}\right)\right|=2|G|\left|C_{A u t(G)}(\beta)\right|$ e que $\left|C_{A u t(G)}(\alpha)\right|=\left|C_{A u t(G)}(\beta)\right|$.

Como consequência dos corolários 4.2.11, 4.2.12, 4.2.17 e 4.2.18, obtemos a ordem de $\operatorname{Half}\left(L_{\alpha}, L_{\beta}\right)$.

Corolário 4.2.20. Com as mesmas notações anteriores, temos que:

a) Se $\beta$ e $\beta J$ não pertencem a $C l(\alpha)$, então $\operatorname{Half}\left(L_{\alpha}, L_{\beta}\right)=\emptyset$

b) Se $\beta$ não pertence a $C l(\alpha)$ e $\beta J$ pertence a $C l(\alpha)$, então $\left|\operatorname{Half}\left(L_{\alpha}, L_{\beta}\right)\right|=2 \cdot|G| .\left|C_{A u t(G)}(\alpha)\right|$

c) Se $\beta$ pertence a $C l(\alpha)$ e $\beta J$ não pertence a $C l(\alpha)$, então $\left|H a l f\left(L_{\alpha}, L_{\beta}\right)\right|=2 \cdot|G| \cdot\left|C_{A u t(G)}(\alpha)\right|$

d) Se $\beta$ e $\beta J$ pertencem a $C l(\alpha)$, então $\left|\operatorname{Half}\left(L_{\alpha}, L_{\beta}\right)\right|=4 .|G| \cdot\left|C_{A u t(G)}(\alpha)\right|$ 


\subsection{O grupo de half-automorfismos de $L_{\alpha}$}

Nesta seção vamos identificar e caracterizar o grupo de half-automorfismos dos loops automórficos diedrais. Lembrando que este grupo é formado por half-automorfismos triviais e não triviais, e que $L_{\alpha}$ denota o loop automórfico diedral $\operatorname{Dih}(\alpha, G)$. Além disso, denotaremos a identidade de $\operatorname{Aut}(G)$ por $I^{\prime}$.

Primeiramente vamos definir os conceitos de produto semidireto entre grupos e o de grupo holomorfo para fixar a notação.

Sejam $G$ e $H$ grupos e $\varphi: G \rightarrow A u t(H)$ um homomorfismo, onde para a pertencente a $G$, denotamos por $\varphi_{a}$ o automorfismo $\varphi(a)$ de $H$. O produto semidireto entre $G$ e $H$ é o grupo denotado por $G \stackrel{\varphi}{\ltimes} H$, formado pelo conjunto $G \times H$ com a operação $\stackrel{\varphi}{*}$ dada por:

$$
(a, b) \stackrel{\varphi}{*}(c, d)=\left(a c, b \varphi_{a}(d)\right)
$$

O grupo holomorfo de um grupo $G$, denotado por $\operatorname{Hol}(G)$, é o produto semidireto $A u t(G) \stackrel{\pi}{\ltimes} G$, onde $\pi$ é a função identidade:

$$
\begin{array}{ccc}
\pi: A u t(G) & \rightarrow \operatorname{Aut}(G) \\
\phi & \mapsto \quad \phi
\end{array}
$$

Agora, como $L_{\alpha}$ é finito, do corolário 3.2 .9 e da proposição 3.3.2 segue que $\operatorname{Half}\left(L_{\alpha}\right)$ é grupo. Este grupo contém o grupo dos automorfismos de $L_{\alpha}$, que foi caracterizado em quase todos os casos no artigo [AV16] através do resultado seguinte.

Proposição 4.3.1. ([AV16] Corollary 5.4) Se $\alpha$ é diferente de $I^{\prime}$ ou se $\alpha=I^{\prime}$ e G não tem período 2, então:

$$
\operatorname{Aut}\left(L_{\alpha}\right) \cong C_{A u t(G)}(\alpha) \stackrel{\pi}{\ltimes} G
$$

onde $C_{\text {Aut }(G)}(\alpha) \stackrel{\pi}{\ltimes} G$ é subgrupo de $\operatorname{Hol}(G)$.

Considere agora o caso onde $\alpha=I^{\prime}$ e $G$ é grupo abeliano de período 2 . Assim $G \cong \prod_{i=1}^{m} C_{2}$, onde $m \geq 1$. É fácil ver que $L_{\alpha}$ é um grupo abeliano de período 2 , e desta forma $L_{\alpha} \cong \prod_{i=1}^{m+1} C_{2}$. Logo, não é difícil ver que neste caso:

$$
\operatorname{Aut}\left(L_{\alpha}\right) \cong G L\left(m+1, \mathbb{Z}_{2}\right)
$$

onde $G L\left(m+1, \mathbb{Z}_{2}\right)$ é o grupo linear geral das matrizes quadradas invertíveis de tamanho $m+1$ com entradas em $\mathbb{Z}_{2}$.

No caso onde $L_{\alpha}$ é comutativo, todo half-automorfismo é um automorfismo, e assim $\operatorname{Half}\left(L_{\alpha}\right)=\operatorname{Half}_{T}\left(L_{\alpha}\right)=\operatorname{Aut}\left(L_{\alpha}\right)$, sendo este último descrito pela proposição 4.3 .1 e pela discussão acima. Assim, de agora em diante, $L_{\alpha}$ será não comutativo, ou equivalentemente, $G$ não terá período 2 .

Seguindo a notação da seção anterior, onde $f^{\prime}$ representa um automorfismo de $G$, vamos definir os seguintes conjuntos: 
$A=\left\{f^{\prime} \in \operatorname{Aut}(G) \mid \alpha=f^{\prime} \alpha f^{\prime-1}\right\}$

$B=\left\{f^{\prime} \in \operatorname{Aut}(G) \mid \alpha J=f^{\prime} \alpha f^{\prime-1}\right\}$

Note que o conjunto $A$ é igual à $C_{A u t(G)}(\alpha)$, o centralizador de $\alpha$ em $A u t(G)$, que é não vazio, pois contém a identidade $I^{\prime}$ de $A u t(G)$. Já o conjunto $B$ é não vazio quando $\alpha J$ pertence a $C l(\alpha)$ (corolário 4.2.11). Além disso, estes conjuntos são disjuntos.

Para os conjuntos $A$ e $B$ temos também o resultado seguinte.

Lema 4.3.2. Temos que:

a) Se $f^{\prime}$ pertence a $A$, então $f^{\prime-1}$ pertence a $A$.

b) Se $f^{\prime}$ pertence a $B$, então $f^{-1}$ pertence a $B$.

c) Se $f^{\prime}$ pertence a $A$ e $g^{\prime}$ pertence a $A$, então $f^{\prime} g^{\prime}$ pertence a $A$.

d) Se $f^{\prime}$ pertence a $A$ e $g^{\prime}$ pertence a $B$, então $f^{\prime} g^{\prime}$ pertence a $B$.

e) Se $f^{\prime}$ pertence a $B$ e $g^{\prime}$ pertence a $A$, então $f^{\prime} g^{\prime}$ pertence a $B$.

f) Se $f^{\prime}$ pertence a $B$ e $g^{\prime}$ pertence a $B$, então $f^{\prime} g^{\prime}$ pertence a $A$.

Demonstração. Os itens a) e c) seguem do fato de $A$ ser subgrupo de $A u t(G)$. Desta forma, serão demonstrados apenas os item b) e d), pois as demonstrações de e) e f) são análogos à de d).

b) Seja $f^{\prime} \in B$. Assim $f^{\prime} \alpha f^{\prime-1}=\alpha J$, o que implica que $\alpha=f^{\prime-1} \alpha J f^{\prime}$. Como $J \in \mathcal{Z}(A u t(G))$ e tem ordem 2 , temos que $\alpha J=f^{\prime-1} \alpha f^{\prime}$. Logo $f^{\prime-1} \in B$.

d) Sejam $f^{\prime} \in A$ e $g^{\prime} \in B$. Assim:

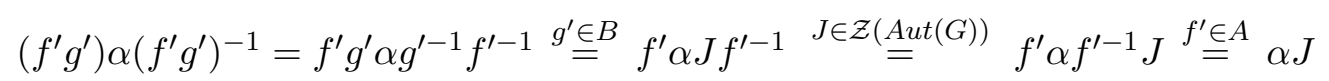

Na seção anterior, para $f^{\prime}$ pertencente a $A u t(G)$, foram construídas extensões $f_{+a}$ e $f_{-a}(a$ pertencente a $G$ ) sobre o conjunto $L_{\alpha}$. Agora denote por $f_{+a}^{*}$ e $f_{-a}^{*}$ estas extensões para o caso onde $f^{\prime}$ pertence a $A$, e denote por $f_{+a}^{\bullet}$ e $f_{-a}^{\bullet}$ estas extensões para o caso onde $f^{\prime}$ pertence a $B$. Com isto, dos teoremas 4.2.10 e 4.2.16 e da proposição 4.2.6 temos:

$$
\begin{aligned}
& \operatorname{Aut}\left(L_{\alpha}\right)=\left\{f_{+a}^{*} \mid f^{\prime} \in A, a \in G\right\} \\
& \operatorname{Half}_{T}\left(L_{\alpha}\right)=\left\{f_{+a}^{*}, f_{-a}^{*} \mid f^{\prime} \in A, a \in G\right\} \\
& \operatorname{Half}_{N}\left(L_{\alpha}\right)=\left\{f_{+a}^{\bullet}, f_{-a}^{\bullet} \mid f^{\prime} \in B, a \in G\right\} \\
& \operatorname{Half}\left(L_{\alpha}\right)=\left\{f_{+a}^{*}, f_{-a}^{*}, g_{+a}^{\bullet}, g_{-a}^{\bullet} \mid f^{\prime} \in A, g^{\prime} \in B, a \in G\right\}
\end{aligned}
$$

Denotando por $(f g)_{+a}^{*}$ e $(f g)_{-a}^{*}$ as extensões da função composta $f^{\prime} g^{\prime}$ pertencente a $A$, e $(f g)_{+a}^{\bullet}$ e $(f g)_{-a}^{\bullet}$ as extensões da função composta $f^{\prime} g^{\prime}$ pertencente a $B$, calcularemos a seguir os produtos dos elementos de $\operatorname{Half}\left(L_{\alpha}\right)$.

Sejam $f^{\prime}$ e $g^{\prime}$ pertencentes a $A$ e $h^{\prime}$ e $k^{\prime}$ pertencentes a $B$. Assim, para $a, b$ e $u$ pertencentes a $G$, e usando o lema 4.3.2, temos:

$$
\begin{aligned}
& f_{+a}^{*} \circ g_{+b}^{*}((0, u))=f_{+a}^{*}\left(g_{+b}^{*}((0, u))\right)=f_{+a}^{*}((0, u))=(0, u) \\
& f_{+a}^{*} \circ g_{+b}^{*}((1, u))=f_{+a}^{*}\left(g_{+b}^{*}((1, u))\right)=f_{+a}^{*}\left(\left(1, b g^{\prime}(u)\right)\right)=\left(1, a f^{\prime}(b) f^{\prime} g^{\prime}(u)\right)
\end{aligned}
$$


$=>f_{+a}^{*} \circ g_{+b}^{*}=(f g)_{+a f^{\prime}(b)}^{*}$

$f_{+a}^{*} \circ g_{-b}^{*}((0, u))=f_{+a}^{*}\left(g_{-b}^{*}((0, u))\right)=f_{+a}^{*}((0, u))=(0, u)$

$f_{+a}^{*} \circ g_{-b}^{*}((1, u))=f_{+a}^{*}\left(g_{-b}^{*}((1, u))\right)=f_{+a}^{*}\left(\left(1, b g^{\prime}\left(u^{-1}\right)\right)\right)=\left(1, a f^{\prime}(b) f^{\prime} g^{\prime}\left(u^{-1}\right)\right)$

$=>f_{+a}^{*} \circ g_{+b}^{*}=(f g)_{-a f^{\prime}(b)}^{*}$

$f_{+a}^{*} \circ k_{+b}^{\bullet}((0, u))=f_{+a}^{*}\left(k_{+b}^{\bullet}((0, u))\right)=f_{+a}^{*}((0, u))=(0, u)$

$f_{+a}^{*} \circ k_{+b}^{\bullet}((1, u))=f_{+a}^{*}\left(k_{+b}^{\bullet}((1, u))\right)=f_{+a}^{*}\left(\left(1, b k^{\prime}(u)\right)\right)=\left(1, a f^{\prime}(b) f^{\prime} k^{\prime}(u)\right)$

$=>f_{+a}^{*} \circ k_{+b}^{\bullet}=(f k)_{+a f^{\prime}(b)}^{\bullet}$

$f_{+a}^{*} \circ k_{-b}^{\bullet}((0, u))=f_{+a}^{*}\left(k_{-b}^{\bullet}((0, u))\right)=f_{+a}^{*}((0, u))=(0, u)$

$f_{+a}^{*} \circ k_{-b}^{\bullet}((1, u))=f_{+a}^{*}\left(k_{-b}^{\bullet}((1, u))\right)=f_{+a}^{*}\left(\left(1, b k^{\prime}\left(u^{-1}\right)\right)\right)=\left(1, a f^{\prime}(b) f^{\prime} k^{\prime}\left(u^{-1}\right)\right)$

$=>f_{+a}^{*} \circ k_{+b}^{\bullet}=(f k)_{-a f^{\prime}(b)}^{\bullet}$

Fazendo as demais composições, obtemos a tabela a seguir, que representa os produtos em $\operatorname{Half}\left(L_{\alpha}\right)$.

\begin{tabular}{|c|c|c|c|c|}
\hline$\circ$ & $g_{+b}^{*}$ & $g_{-b}^{*}$ & $k_{+b}^{\bullet}$ & $k_{-b}^{\bullet}$ \\
\hline$f_{+a}^{*}$ & $(f g)_{+a f^{\prime}(b)}^{*}$ & $(f g)_{-a f^{\prime}(b)}^{*}$ & $(f k)_{+a f^{\prime}(b)}^{\bullet}$ & $(f k)_{-a f^{\prime}(b)}^{\bullet}$ \\
\hline$f_{-a}^{*}$ & $(f g)_{-a f^{\prime}\left(b^{-1}\right)}^{*}$ & $(f g)_{+a f^{\prime}\left(b^{-1}\right)}^{*}$ & $(f k)_{-a f^{\prime}\left(b^{-1}\right)}^{\bullet}$ & $(f k)_{+a f^{\prime}\left(b^{-1}\right)}^{\bullet}$ \\
\hline$h_{+a}^{\bullet}$ & $(h g)_{+a h^{\prime}(b)}^{\bullet}$ & $(h g)_{-a h^{\prime}(b)}^{\bullet}$ & $(h k)_{+a h^{\prime}(b)}^{*}$ & $(h k)_{-a h^{\prime}(b)}^{*}$ \\
\hline$h_{-a}^{\bullet}$ & $(h g)_{-a h^{\prime}\left(b^{-1}\right)}^{\bullet}$ & $(h g)_{+a h^{\prime}\left(b^{-1}\right)}^{\bullet}$ & $(h k)_{-a h^{\prime}\left(b^{-1}\right)}^{*}$ & $(h k)_{+a h^{\prime}\left(b^{-1}\right)}^{*}$ \\
\hline
\end{tabular}

A partir desta tabela, vemos que $I_{+e}^{*}$ é a identidade de $\operatorname{Half}\left(L_{\alpha}\right)$, onde $I^{\prime}$ é a identidade de $\operatorname{Aut}(G)$ e $e$ é a identidade de $G$. Além disso, sendo $f_{+x}^{-1 *}$ e $f_{-x}^{-1 *}$ as extensões de $f^{\prime-1}$ pertencente a $A$ e $f_{+x}^{-1 \bullet}$ e $f_{-x}^{-1 \bullet}$ as extensões de $f^{\prime-1}$ pertencente a $B$, podemos obter os inversos dos elementos de $\operatorname{Half}\left(L_{\alpha}\right)$ :

$$
\left(f_{+a}^{*}\right)^{-1}=f_{+f^{\prime-1}\left(a^{-1}\right)}^{-1 *},\left(f_{-a}^{*}\right)^{-1}=f_{-f^{\prime-1}(a)}^{-1 *},\left(f_{+a}^{\bullet}\right)^{-1}=f_{+f^{\prime-1}\left(a^{-1}\right)}^{-1 \bullet},\left(f_{-a}^{\bullet}\right)^{-1}=f_{-f^{\prime-1}(a)}^{-1 \bullet}
$$

Agora defina a seguinte função:

$$
\begin{aligned}
\varphi: \operatorname{Aut}\left(L_{\alpha}\right) & \rightarrow \operatorname{Aut}\left(L_{\alpha}\right) \\
f_{+a}^{*} & \mapsto f_{+a^{-1}}^{*}
\end{aligned}
$$

É fácil ver que $\varphi$ é uma bijeção. Além disso, para $f_{+a}^{*}$ e $g_{+b}^{*}$ pertencentes a $A u t\left(L_{\alpha}\right)$, temos:

$$
\begin{aligned}
& \varphi\left(f_{+a}^{*} \circ g_{+b}^{*}\right)=\varphi\left((f g)_{+a f^{\prime}(b)}^{*}\right)=(f g)_{+f^{\prime}\left(b^{-1}\right) a^{-1}}^{*} \stackrel{G \text { comut. }}{=}(f g)_{+a^{-1} f^{\prime}\left(b^{-1}\right)}^{*}=f_{+a^{-1}}^{*} \circ g_{+b^{-1}}^{*}= \\
& =\varphi\left(f_{+a}^{*}\right) \circ \varphi\left(g_{+b}^{*}\right)
\end{aligned}
$$

Assim $\varphi$ é um automorfismo.

Além disso, temos que $\varphi$ tem ordem 2. Logo podemos definir o produto semidireto

$$
C_{2} \stackrel{\sigma}{\ltimes} \operatorname{Aut}\left(L_{\alpha}\right)
$$

onde:

$$
\begin{array}{rccc}
\sigma: & C_{2} & \rightarrow & \operatorname{Aut}\left(\operatorname{Aut}\left(L_{\alpha}\right)\right) \\
1 & \mapsto & I \\
2 & \mapsto & \varphi
\end{array}
$$


sendo $I$ a identidade de $\operatorname{Aut}\left(\operatorname{Aut}\left(L_{\alpha}\right)\right)$. Com isso, a tabela de multiplicação desse produto semidireto é representada pela tabela seguinte.

\begin{tabular}{|c|c|c|}
\hline $\begin{array}{c}\sigma \\
*\end{array}$ & $\left(0, g_{+b}^{*}\right)$ & $\left(1, g_{+b}^{*}\right)$ \\
\hline$\left(0, f_{+a}^{*}\right)$ & $\left(0, f_{+a}^{*} \circ g_{+b}^{*}\right)$ & $\left(1, f_{+a}^{*} \circ g_{+b}^{*}\right)$ \\
\hline$\left(1, f_{+a}^{*}\right)$ & $\left(1, f_{+a}^{*} \circ g_{+b^{-1}}^{*}\right)$ & $\left(0, f_{+a}^{*} \circ g_{+b^{-1}}^{*}\right)$ \\
\hline
\end{tabular}

Teorema 4.3.3. $\operatorname{Half}_{T}\left(L_{\alpha}\right) \cong C_{2} \stackrel{\sigma}{\ltimes} \operatorname{Aut}\left(L_{\alpha}\right) \cong C_{2} \stackrel{\sigma}{\ltimes}\left(C_{A u t(G)}(\alpha) \stackrel{\pi}{\ltimes} G\right)$

Demonstração. Defina a função:

$$
\begin{aligned}
\psi: \operatorname{Half}_{T}\left(L_{\alpha}\right) & \rightarrow & C_{2} \stackrel{\sigma}{\ltimes} \operatorname{Aut}\left(L_{\alpha}\right) \\
f_{+a}^{*} & \mapsto & \left(0, f_{+a}^{*}\right) \\
f_{-a}^{*} & \mapsto & \left(1, f_{+a}^{*}\right)
\end{aligned}
$$

Claramente $\psi$ é bijetora. Agora vamos mostrar que $\psi$ é um homomorfismo. Para isso, analisaremos os quatro casos seguintes.

(i) Sejam $f_{+a}^{*}, g_{+b}^{*} \in \operatorname{Half}_{T}\left(L_{\alpha}\right)$. Assim:

$$
\begin{aligned}
& \psi\left(f_{+a}^{*} \circ g_{+b}^{*}\right)=\psi\left((f g)_{+a f^{\prime}(b)}^{*}\right)=\left(0,(f g)_{+a f^{\prime}(b)}^{*}\right)=\left(0, f_{+a}^{*} \circ g_{+b}^{*}\right)=\left(0, f_{+a}^{*}\right)^{\sigma} *\left(0, g_{+b}^{*}\right)= \\
& =\psi\left(f_{+a}^{*}\right)^{\sigma} * \psi\left(g_{+b}^{*}\right)
\end{aligned}
$$

(ii) Sejam $f_{+a}^{*}, g_{-b}^{*} \in \operatorname{Half}_{T}\left(L_{\alpha}\right)$. Assim:

$\psi\left(f_{+a}^{*} \circ g_{-b}^{*}\right)=\psi\left((f g)_{-a f^{\prime}(b)}^{*}\right)=\left(1,(f g)_{+a f^{\prime}(b)}^{*}\right)=\left(1, f_{+a}^{*} \circ g_{+b}^{*}\right)=\left(0, f_{+a}^{*}\right)^{\sigma}{ }^{*}\left(0, g_{+b}^{*}\right)=$

$=\psi\left(f_{+a}^{*}\right)^{\sigma} * \psi\left(g_{+b}^{*}\right)$

(iii) Sejam $f_{-a}^{*}, g_{+b}^{*} \in \operatorname{Half}_{T}\left(L_{\alpha}\right)$. Assim:

$\psi\left(f_{-a}^{*} \circ g_{+b}^{*}\right)=\psi\left((f g)_{-a f^{\prime}\left(b^{-1}\right)}^{*}\right)=\left(1,(f g)_{+a f^{\prime}\left(b^{-1}\right)}^{*}\right)=\left(1, f_{+a}^{*} \circ g_{+b^{-1}}^{*}\right)=\left(1, f_{+a}^{*}\right)^{\sigma} *\left(0, g_{+b}^{*}\right)=$ $=\psi\left(f_{-a}^{*}\right) \stackrel{\sigma}{*} \psi\left(g_{+b}^{*}\right)$

(iv) Sejam $f_{-a}^{*}, g_{-b}^{*} \in \operatorname{Half}_{T}\left(L_{\alpha}\right)$. Assim:

$$
\begin{aligned}
& \psi\left(f_{-a}^{*} \circ g_{-b}^{*}\right)=\psi\left((f g)_{+a f^{\prime}\left(b^{-1}\right)}^{*}\right)=\left(0,(f g)_{+a f^{\prime}\left(b^{-1}\right)}^{*}\right)=\left(0, f_{+a}^{*} \circ g_{+b^{-1}}^{*}\right)=\left(1, f_{+a}^{*}\right)^{\sigma}\left(1, g_{+b}^{*}\right)= \\
& =\psi\left(f_{-a}^{*}\right) \stackrel{\sigma}{*} \psi\left(g_{-b}^{*}\right)
\end{aligned}
$$

Portanto $\psi$ é isomorfismo. O restante do teorema segue da proposição 4.3.1.

Teorema 4.3.4. Com as mesmas notações anteriores, valem:

a) Se $\alpha J$ não pertence a $C l(\alpha)$, então $\operatorname{Half}\left(L_{\alpha}\right)=\operatorname{Half}_{T}\left(L_{\alpha}\right) \cong C_{2} \stackrel{\sigma}{\ltimes}\left(C_{A u t(G)}(\alpha) \stackrel{\pi}{\ltimes} G\right)$

b) Se $\alpha J$ pertence a $C l(\alpha)$, então Half $\left(L_{\alpha}\right) \cong C_{2} \times \operatorname{Half}_{T}\left(L_{\alpha}\right) \cong C_{2} \times\left(C_{2} \stackrel{\sigma}{\ltimes}\left(C_{\text {Aut }(G)}(\alpha) \stackrel{\pi}{\ltimes} G\right)\right)$ 
Demonstração. a) Se $\alpha J \notin C l(\alpha)$, do corolário 4.2.11 segue que $\operatorname{Half}_{N}\left(L_{\alpha}\right)$ é um conjunto vazio, $\operatorname{assim} \operatorname{Half}\left(L_{\alpha}\right)=\operatorname{Half}_{T}\left(L_{\alpha}\right)$, e o restante segue do teorema 4.3.3.

b) Defina a função:

$$
\begin{array}{rlc}
\psi: \quad \operatorname{Half}_{(}\left(L_{\alpha}\right) & \rightarrow & C_{2} \times \operatorname{Half}_{T}\left(L_{\alpha}\right) \\
f_{+a}^{*} & \mapsto & \left(0, f_{+a}^{*}\right) \\
f_{-a}^{*} & \mapsto & \left(0, f_{-a}^{*}\right) \\
f_{+a}^{\bullet} & \mapsto & \left(1, f_{+a}^{*}\right) \\
f_{-a}^{\bullet} & \mapsto & \left(1, f_{-a}^{*}\right)
\end{array}
$$

Claramente $\psi$ é bijetora. Agora vamos mostrar que $\psi$ é um homomorfismo. Para isso, analisaremos os dezesseis casos de produtos de $C_{2} \times \operatorname{Half}_{T}\left(L_{\alpha}\right)$.

Primeiro, a partir da tabela que representa os produtos em $\operatorname{Half}\left(L_{\alpha}\right)$, obtemos a seguinte tabela para $\operatorname{Half}_{T}\left(L_{\alpha}\right)$ :

\begin{tabular}{|c|c|c|}
\hline$\circ$ & $g_{+b}^{*}$ & $g_{-b}^{*}$ \\
\hline$f_{+a}^{*}$ & $(f g)_{+a f^{\prime}(b)}^{*}$ & $(f g)_{-a f^{\prime}(b)}^{*}$ \\
\hline$f_{-a}^{*}$ & $(f g)_{-a f^{\prime}\left(b^{-1}\right)}^{*}$ & $(f g)_{+a f^{\prime}\left(b^{-1}\right)}^{*}$ \\
\hline
\end{tabular}

Agora, sejam $f_{+a}^{*}, f_{-a}^{*}, g_{+b}^{*}, g_{-b}^{*}, h_{+a}^{*}, h_{-a}^{*}, k_{+b}^{*}, k_{-b}^{*} \in \operatorname{Half}_{T}\left(L_{\alpha}\right)$. Assim a tabela que representa o produto em $C_{2} \times \operatorname{Half}_{T}\left(L_{\alpha}\right)$ é dada por:

\begin{tabular}{|c|c|c|c|c|}
\hline$\cdot$ & $\left(0, g_{+b}^{*}\right)$ & $\left(0, g_{-b}^{*}\right)$ & $\left(1, k_{+b}^{*}\right)$ & $\left(1, k_{-b}^{*}\right)$ \\
\hline$\left(0, f_{+a}^{*}\right)$ & $\left(0,(f g)_{+a f^{\prime}(b)}^{*}\right)$ & $\left(0,(f g)_{-a f^{\prime}(b)}^{*}\right)$ & $\left(1,(f k)_{+a f^{\prime}(b)}^{*}\right)$ & $\left(1,(f k)_{-a f^{\prime}(b)}^{*}\right)$ \\
\hline$\left(0, f_{-a}^{*}\right)$ & $\left(0,(f g)_{-a f^{\prime}\left(b^{-1}\right)}^{*}\right)$ & $\left(0,(f g)_{+a f^{\prime}\left(b^{-1}\right)}^{*}\right)$ & $\left(1,(f k)_{-a f^{\prime}\left(b^{-1}\right)}^{*}\right)$ & $\left(1,(f k)_{+a f^{\prime}\left(b^{-1}\right)}^{*}\right)$ \\
\hline$\left(1, h_{+a}^{*}\right)$ & $\left(1,(h g)_{+a h^{\prime}(b)}^{*}\right)$ & $\left(1,(h g)_{-a h^{\prime}(b)}^{*}\right)$ & $\left(0,(h k)_{+a h^{\prime}(b)}^{*}\right)$ & $\left(0,(h k)_{-a h^{\prime}(b)}^{*}\right)$ \\
\hline$\left(1, h_{-a}^{*}\right)$ & $\left(1,(h g)_{-a h^{\prime}\left(b^{-1}\right)}^{*}\right)$ & $\left(1,(h g)_{+a h^{\prime}\left(b^{-1}\right)}^{*}\right)$ & $\left(0,(h k)_{-a h^{\prime}\left(b^{-1}\right)}^{*}\right)$ & $\left(0,(h k)_{+a h^{\prime}\left(b^{-1}\right)}^{*}\right)$ \\
\hline
\end{tabular}

Comparando-se a tabela acima com a tabela que representa os produtos em $\operatorname{Half}\left(L_{\alpha}\right)$, vemos que $\psi$ é um isomorfismo. O restante segue do teorema 4.3.3.

Terminaremos essa seção com um exemplo. Construiremos um loop $L_{\alpha}$ onde $\operatorname{Half}\left(L_{\alpha}\right)$ é diferente de $\operatorname{Half}_{T}\left(L_{\alpha}\right)$, ou seja, o grupo abeliano $G$ é tal que seu período é diferente de 2 e existe $\alpha$ pertencente a $A u t(G)$ tal que $\alpha J$ pertence a $C l(\alpha)$. Caso o grupo $A u t(G)$ seja abeliano, então não existe $\alpha$ pertencente a $A u t(G)$ tal que $\alpha J$ pertence a $C l(\alpha)$. Como o grupo de automorfismos de um grupo cíclico sempre é abeliano, então o grupo de menor ordem candidato a este exemplo é o grupo $G=C_{4} \times C_{2}$.

Exemplo 4.3.5. Seja $G=C_{4} \times C_{2}$, e considere sua tabela de multiplicação:

\begin{tabular}{|c|c|c|c|c|c|c|c|c|}
\hline$*$ & 1 & 2 & 3 & 4 & 5 & 6 & 7 & 8 \\
\hline 1 & 1 & 2 & 3 & 4 & 5 & 6 & 7 & 8 \\
\hline 2 & 2 & 4 & 5 & 6 & 7 & 1 & 8 & 3 \\
\hline 3 & 3 & 5 & 1 & 7 & 2 & 8 & 4 & 6 \\
\hline 4 & 4 & 6 & 7 & 1 & 8 & 2 & 3 & 5 \\
\hline 5 & 5 & 7 & 2 & 8 & 4 & 3 & 6 & 1 \\
\hline 6 & 6 & 1 & 8 & 2 & 3 & 4 & 5 & 7 \\
\hline 7 & 7 & 8 & 4 & 3 & 6 & 5 & 1 & 2 \\
\hline 8 & 8 & 3 & 6 & 5 & 1 & 7 & 2 & 4 \\
\hline
\end{tabular}

É um fato bem conhecido que seu grupo de automorfismos tem ordem 8 e tem a seguinte apresentação em termos de permutações: 


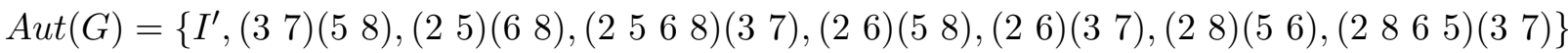

Note que $A u t(G)$ é isomorfo ao grupo diedral de ordem 8 e que a função $J$ é a permutação (2 6$)(58)$.

Seja $\alpha=\left(\begin{array}{l}3 \\ 7\end{array}\right)(58)$. Assim, sendo $f^{\prime}=(25)(68)$, temos que $\alpha J=f^{\prime} \alpha f^{\prime-1}$. Além disso, os conjuntos $A$ e $B$ são dados por:

$A=\left\{I^{\prime},(37)(58),(26)(58),(26)(37)\right\}$

$B=\{(25)(68),(2568)(37),(28)(56),(2865)(37)\}$

Com isso, $\left|C_{A u t(G)}(\alpha)\right|=|A|=4$ e, para o $A$-loop $L_{\alpha}=\operatorname{Dih}\left(C_{4} \times C_{2},(37)(58)\right)$, temos:

$\left|\operatorname{Half}\left(L_{\alpha}\right)\right|=4 .|G| \cdot\left|C_{\text {Aut }(G)}(\alpha)\right|=128$

$\left|\operatorname{Half}_{T}\left(L_{\alpha}\right)\right|=2 .|G| \cdot\left|C_{\text {Aut }(G)}(\alpha)\right|=64$

$\left|A u t\left(L_{\alpha}\right)\right|=|G| .\left|C_{\text {Aut }(G)}(\alpha)\right|=32$

Agora vamos exibir exemplos de half-automorfismos. Sejam os elementos $f^{\prime}=(26)(37)$ pertencente a $A$ e $g^{\prime}=\left(\begin{array}{ll}2 & 8\end{array}\right)\left(\begin{array}{l}5 \\ 6\end{array}\right)$ pertencente a $B$. Assim a extensão $f_{+1}^{*}$ é um automorfismo, a extensão $f_{-1}^{*}$ é um anti-automorfismo e a extensão $g_{+1}^{\bullet}$ é um half-automorfismo não trivial de $L_{\alpha}$. Suas expressões em termos de permutações são:

$$
\begin{aligned}
& f_{+1}^{*}=((0,2)(0,6))((0,3)(0,7))((1,2)(1,6))((1,3)(1,7)) \\
& f_{-1}^{*}=((0,2)(0,6))((0,3)(0,7))((1,3)(1,7))((1,5)(1,8)) \\
& g_{+1}^{\bullet}=((0,2)(0,8))((0,5)(0,6))((1,2)(1,8))((1,5)(1,6))
\end{aligned}
$$




\section{Capítulo 5}

\section{Half-automorfismos de loops automórficos de Lie}

Em [Wri69] C.R.B. Wright obteve um loop a partir de uma álgebra definindo uma nova operação entre seus elementos. Ele considerou $(A,+, \cdot)$ uma álgebra, e definiu uma operação $*$ da seguinte forma: $a * b=a+b-a \cdot b$. Seguindo a construção de Wright, M.K. Kinyon, K. Kunen, J.D. Phillips e P. Vojtěchovský [KKPV16] construíram uma classe de $A$-loops a partir de anéis de Lie. Aqui denominamos esta classe de loop automórfico de Lie. Em [GA17] os autores M.L. Merlini Giuliani e G.S. Anjos obtiveram o grupo de automorfismos de uma classe destes loops de ordem ímpar da forma $n^{3}$. Neste capítulo estudamos a existência de half-automorfismos não triviais em loops automórficos de Lie. Introduzimos os conceitos necessários para provar o resultado principal: em loops automórficos de Lie de ordem ímpar, todo half-automorfismo é trivial.

\subsection{Loop automórfico de Lie}

Definição 5.1.1. Uma tripla ordenada $(Q,+,[.,]$.$) , onde Q$ é um conjunto não vazio e + e [., .] são operações binárias definidas em $Q$, é um anel de Lie se para todo $x, y, z$ e $w$ pertencentes a $Q$ temos:
a) $x+(y+z)=(x+y)+z$,
b) $x+y=y+x$,
c) Existe 0 pertencente a $Q$ tal que $0+a=a$, para todo $a$ pertencente a $Q$,
d) Para todo $a$ pertencente a $Q$, existe $-a$ pertencente a $Q$ tal que $a+(-a)=0$,
e) $[x+y, z+w]=[x, z]+[x, w]+[y, z]+[y, w]$,
f) $[x, x]=0$,
g) $[x,[y, z]]+[y,[z, x]]+[z,[x, y]]=0$ (Identidade de Jacobi).

Observe que num anel de Lie $(Q,+,[.,]$.$) , o par ordenado (Q,+)$ é um grupo abeliano com elemento identidade 0 . Além disso, para $x$ e $y$ pertencentes a $Q$, usando e) e f) temos:

$$
0=[x+y, x+y]=[x, x]+[x, y]+[y, x]+[y, y]=[x, y]+[y, x]
$$

Assim em anéis de Lie é válida a seguinte relação, chamada de propriedade anti-simétrica:

$$
[x, y]=-[y, x], \text { para todo } x, y \in Q
$$

Outras propriedades básicas válidas para anéis de Lie são:

(i) O elemento 0 é único;

(ii) $[0, x]=[x, 0]=0$, para todo $x$ pertencente a $Q$. 
Definição 5.1.2. Dizemos que um subconjunto não vazio $H$ de um anel de Lie $(Q,+,[.,]$.$) é um$ subanel de Lie se, para todo $x$ e $y$ pertencentes a $H$, temos que $x-y$ e $[x, y]$ pertencem a $H$.

Agora, seja $(Q,+,[.,]$.$) um anel de Lie. Defina *$ em $Q$ por:

$$
x * y=x+y-[x, y]
$$

Sejam as seguintes condições sobre $(Q,+,[.,]$.$) :$

As aplicações $y \mapsto y+[y, x]$ são invertíveis para todo $x$ pertencente a $Q$;

$[[Q, x],[Q, x]]=0$ para todo $x$ pertencente a $Q ;$

$[[x, y],[z, w]]=0$ para todo $x, y, z$ e $w$ pertencentes a $Q$;

Note que a condição 5.4 implica a condição 5.3. O contrário não necessariamente é válido.

As três proposições seguintes podem ser encontradas em [KKPV16]:

Proposição 5.1.3. (Lema 5.1) $(Q, *)$ é loop com elemento identidade 0 se, e somente se, o anel de Lie $(Q,+,[.,]$.$) satisfaz 5.2.$

Proposição 5.1.4. (Prop. 5.2) Seja $(Q,+,[.,]$.$) um anel de Lie satisfazendo 5.2 e 5.3. Então$ $(Q, *)$ é A-loop e:

$$
\begin{aligned}
C(Q) & =\{a \in Q \mid 2[a, x]=0 \quad \forall x \in Q\} \\
N_{\lambda}(Q) & =\{a \in Q \mid[x,[y, a]]=0 \quad \forall x, y \in Q\} \\
N_{\mu}(Q) & =\{a \in Q \mid[a,[x, y]]=0 \quad \forall x, y \in Q\}
\end{aligned}
$$

Em particular, $(Q, *)$ é grupo se, e somente se, $[[x, y], z]=0$ para todo $x, y$ e z pertencentes a $Q$.

Chamaremos os A-loops do tipo $(Q, *)$ de loops automórficos de Lie.

Proposição 5.1.5. (Lema 5.8) Seja $(Q,+,[.,]$.$) um anel de Lie de ordem de impar. Então Q$ satisfaz 5.3 se, e somente se, satisfaz 5.4 .

Pela proposição 5.1.4 temos que se um anel de Lie $Q$ satisfaz 5.2 e 5.3 e é de característica 2, então $C(Q)=Q$, implicando que $(Q, *)$ é $A$-loop comutativo.

Se um anel de Lie $(Q,+,[.,]$.$) é de ordem ímpar, por (Q,+)$ ser grupo abeliano segue que ele não contém nenhum elemento de ordem par. Logo, a seguinte relação é válida:

$$
2 x=0<=>x=0
$$

Esta relação será útil para provar alguns itens da proposição a seguir e no decorrer do capítulo. 
Proposição 5.1.6. Sejam $(Q,+,[.,]$.$) um anel de Lie de ordem impar satisfazendo 5.2 e 5.3. En-$ tão em $(Q, *)$ as seguintes relações são válidas, para todo $x, y$ e z pertencentes a $Q$ e para todo $n$ pertencente a $N_{\mu}(Q)$ :

a) $x * y=y * x$ se, e somente se, $[x, y]=0$.

b) $x *(x * y)=(y * x) * x$ se, e somente se, $x * y=y * x$.

c) $x *(y * n)=y *(x * n)$ se, e somente se, $x * y=y * x$.

d) $(n * y) * x=y *(x * n)$ se, e somente se, $[n, x+y]=0$.

e) $(n * x) *(n * y)=(n * y) *(n * x)$ se, e somente se, $(x * n) *(y * n)=(y * n) *(x * n)$, e estas duas equações são válidas se, e somente se, $x *(n * y)=y *(n * x)$.

f) $x *(y * z)=(x * y) * z$ se, e somente se, $[y,[z, x]]=0$.

Demonstração. Sejam $x, y, z \in Q$ e $n \in N_{\mu}(Q)$.

a) Calculando $x * y$ e $y * x$, obtemos:

$x * y=x+y-[x, y]$

$y * x=y+x-[y, x]=x+y+[x, y]$

Assim:

$$
x * y=y * x<=>2[x, y]=0
$$

Logo, da expressão 5.8 segue que $x * y=y * x$ se, e somente se, $[x, y]=0$.

b) Calculando $x *(x * y)$ e $(y * x) * x$, obtemos:

$$
\begin{aligned}
x *(x * y) & =x *(x+y-[x, y]) \\
& =2 x+y-[x, y]-[x, x+y-[x, y]] \\
& =2 x+y-2[x, y]+[x,[x, y]] \\
(y * x) * x & =(y+x-[y, x]) * x \\
& =2 x+y-[y, x]-[y+x-[y, x], x] \\
& =2 x+y+2[x, y]+[x,[x, y]]
\end{aligned}
$$

Desta forma:

$$
x *(x * y)=(y * x) * x<=>4[x, y]=0
$$

Com isso, usando o item a) e a expressão 5.8 temos que $x *(x * y)=(y * x) * x$ se, e somente se $x * y=y * x$.

c) Da identidade de Jacobi, temos que:

$$
[x,[y, n]]+[y,[n, x]]+[n,[x, y]]=0
$$

Como $n$ está no núcleo central, da expressão 5.7 temos que $[n,[x, y]]=0$. Assim:

$$
[x,[y, n]]+[y,[n, x]]=0
$$

Agora, calculando $x *(y * n)$ e $y *(x * n)$, obtemos:

$$
\begin{aligned}
x *(y * n) & =x *(y+n-[y, n]) \\
& =x+y+n-[y, n]-[x, y+n-[y, n]] \\
& =x+y+n-[y, n]-[x, y]-[x, n]+[x,[y, n]]
\end{aligned}
$$




$$
\begin{aligned}
y *(x * n) & =y *(x+n-[x, n]) \\
& =x+y+n-[x, n]-[y, x+n-[x, n]] \\
& =x+y+n-[x, n]-[y, x]-[y, n]+[y,[x, n]]
\end{aligned}
$$

Da equação 5.9 segue que $[x,[y, n]]=[y,[x, n]]$. Assim:

$$
x *(y * n)=y *(x * n)<=>2[x, y]=0
$$

Logo, usando o item a) e a expressão 5.8 temos que $x *(y * n)=y *(x * n)$ se, e somente se $x * y=y * x$.

d) Calculando $(n * y) * x$ e $y *(x * n)$, obtemos:

$$
\begin{aligned}
& (n * y) * x=(n+y-[n, y]) * x \\
& =n+x+y-[n, y]-[n+y-[n, y], x] \\
& =n+x+y-[n, y]-[n, x]-[y, x]+[[n, y], x] \\
& y *(x * n)=y *(x+n-[x, n]) \\
& =n+x+y-[x, n]-[y, x+n-[x, n]] \\
& =n+x+y-[x, n]-[y, x]-[y, n]+[y,[x, n]]
\end{aligned}
$$

Desta forma:

$$
(n * y) * x=y *(x * n)<=>-[n, x+y]+[[n, y], x]=[n, x+y]+[y,[x, n]]
$$

Rearranjando os termos da equação da direita, temos:

$$
(n * y) * x=y *(x * n)<=>2[n, x+y]=[y,[n, x]]+[x,[y, n]]
$$

Da equação 5.9 segue que $2[n, x+y]=0$. Assim, usando a expressão 5.8 obtemos que $(n * y) * x=y *(x * n)$ se, e somente se, $[n, x+y]=0$.

e) Calculando $x *(n * y)$ e $y *(n * x)$, obtemos:

$$
\begin{aligned}
x *(n * y) & =x *(n+y-[n, y]) \\
& =x+n+y-[n, y]-[x, n+y-[n, y]] \\
& =x+n+y-[n, y]-[x, n]-[x, y]+[x,[n, y]] \\
y *(n * x) & =y *(n+x-[n, x]) \\
& =x+n+y-[n, x]-[y, n+x-[n, x]] \\
& =x+n+y-[n, x]-[y, n]-[y, x]+[y,[n, x]]
\end{aligned}
$$

Assim:

$x *(n * y)=y *(n * x)<=>[n, x-y]-[x, y]+[x,[n, y]]=-[n, x-y]-[y, x]+[y,[n, x]]$

Rearranjando os termos da equação da direita, temos:

$$
x *(n * y)=y *(n * x)<=>2[n, x-y]-2[x, y]=[x,[y, n]]+[y,[n, x]]
$$

Da equação 5.9 segue que $2[n, x-y]-2[x, y]=0$. Assim, usando a expressão 5.8 obtemos:

$$
x *(n * y)=y *(n * x)<=>[n, x-y]=[x, y]
$$

Agora, de a) temos: 


$$
\begin{array}{llll}
(n * x) *(n * y)=(n * y) *(n * x) & <=> & {[n * x, n * y]=0} \\
(x * n) *(y * n) & =(y * n) *(x * n) & <=> & {[x * n, y * n]=0}
\end{array}
$$

Calculando $[n * x, n * y]$ e $[x * n, y * n]$ obtemos:

$$
\begin{aligned}
{[n * x, n * y] } & =[n+x-[n, x], n+y-[n, y]] \\
& =[n, y]+[x, n]+[x, y]-[x,[n, y]]-[[n, x], y]+[[n, x],[n, y]] \\
& =[x, y]-[n, x-y]+([x,[y, n]]+[y,[n, x]])+[[n, x],[n, y]] \\
{[x * n, y * n] } & =[x+n-[x, n], y+n-[y, n]] \\
& =[x, y]+[x, n]+[n, y]-[x,[y, n]]-[[x, n], y]+[[x, n],[y, n]] \\
& =[x, y]-[n, x-y]-([x,[y, n]]+[y,[n, x]])+[[x, n],[y, n]]
\end{aligned}
$$

Como $Q$ satisfaz 5.3 , temos que $[[n, x],[n, y]]$ e $[[x, n],[y, n]]$ são nulos. Além disso, da equação 5.9 segue que $[x,[y, n]]+[y,[n, x]]=0$. Logo:

$[n * x, n * y]=[x, y]-[n, x-y]$

$[x * n, y * n]=[x, y]-[n, x-y]$

Assim, das equações 5.11 temos:

$$
\begin{array}{llll}
(n * x) *(n * y)=(n * y) *(n * x) & <=> & {[n, x-y]=[x, y]} \\
(x * n) *(y * n)=(y * n) *(x * n) & <=> & {[n, x-y]=[x, y]}
\end{array}
$$

Logo, $(n * x) *(n * y)=(n * y) *(n * x)$ se, e somente se, $(x * n) *(y * n)=(y * n) *(x * n)$, e da equação 5.10 segue que estas equações são equivaletes à $x *(n * y)=y *(n * x)$.

f) Calculando $x *(y * z)$ e $(x * y) * z$, obtemos:

$$
\begin{aligned}
x *(y * z) & =x *(y+z-[y, z]) \\
& =x+y+z-[x, y]-[x, z]-[y, z]+[x,[y, z]] \\
(x * y) * z & =(x+y-[x, y]) * z \\
& =x+y+z-[x, y]-[x, z]-[y, z]+[[x, y], z]
\end{aligned}
$$

Assim:

$$
x *(y * z)=(x * y) * z<=>[x,[y, z]]=[[x, y], z]
$$

Rearranjando os termos da equação da direita, temos:

$$
x *(y * z)=(x * y) * z<=>[x,[y, z]]+[z,[x, y]]=0
$$

Logo, utilizando a identidade de Jacobi, obtemos que $x *(y * z)=(x * y) * z$ se, e somente se, $[y,[z, x]]=0$.

Os resultados da proposição 5.1.6 serão importantes nas demonstrações no decorrer do capítulo. Além disso sabemos que se $(Q, *)$ é um loop associativo, então todo half-automorfismo é trivial, portanto excluiremos este caso da nossa análise. Assim, de agora em diante considere o anel de Lie $(Q,+,[.,]$.$) finito de ordem ímpar, que satisfaça as condições 5.2$ e 5.3 (e por consequência 5.4) e que seja tal que $(Q, *)$ é não associativo. 
Primeiramente daremos um exemplo de um anel de Lie nestas condições. Tal exemplo foi estudado com mais detalhes em [GA17].

Exemplo 5.1.7. Seja $n \in \mathbb{N}$, com $n>1$. Defina o conjunto $P\left(\mathbb{Z}_{n}\right)$ por:

$$
P\left(\mathbb{Z}_{n}\right)=\left\{\left[\begin{array}{ccc}
0 & a & b \\
0 & 0 & 0 \\
-b & c & 0
\end{array}\right] \mid a, b, c \in \mathbb{Z}_{n}\right\}
$$

É fácil ver que $P\left(\mathbb{Z}_{n}\right)$ é fechado para somas e subtrações usuais em matrizes. O colchete usual de matrizes, $[x, y]=x y-y x$, é dado por:

$$
\left[\left[\begin{array}{ccc}
0 & a & b \\
0 & 0 & 0 \\
-b & c & 0
\end{array}\right],\left[\begin{array}{ccc}
0 & a^{\prime} & b^{\prime} \\
0 & 0 & 0 \\
-b^{\prime} & c^{\prime} & 0
\end{array}\right]\right]=\left[\begin{array}{ccc}
0 & b c^{\prime}-b^{\prime} c & 0 \\
0 & 0 & 0 \\
0 & -b a^{\prime}+b^{\prime} a & 0
\end{array}\right]
$$

Assim $P\left(\mathbb{Z}_{n}\right)$ é subanel de Lie do anel de Lie das matrizes de traço zero, cuja expressão é a seguinte:

$$
S L\left(3, \mathbb{Z}_{n}\right)=\left\{A \in M\left(3, \mathbb{Z}_{n}\right) \mid \operatorname{tr}(A)=0\right\}
$$

Da expressão para o colchete dada anteriormente segue também que $P\left(\mathbb{Z}_{n}\right)$ satisfaz a condição 5.4.

Pela definição da operação $*$, temos que o produto em $P\left(\mathbb{Z}_{n}\right)$ é dado pela seguinte expressão:

$$
\left[\begin{array}{ccc}
0 & a & b \\
0 & 0 & 0 \\
-b & c & 0
\end{array}\right] *\left[\begin{array}{ccc}
0 & a^{\prime} & b^{\prime} \\
0 & 0 & 0 \\
-b^{\prime} & c^{\prime} & 0
\end{array}\right]=\left[\begin{array}{ccc}
0 & a+a^{\prime}-b c^{\prime}+b^{\prime} c & b+b^{\prime} \\
0 & 0 & 0 \\
-b-b^{\prime} & c+c^{\prime}+b a^{\prime}-b^{\prime} a & 0
\end{array}\right]
$$

No caso onde $n=\prod_{i=1}^{m} p_{i}^{r_{i}}$ com $p_{i}$ primo satisfazendo $p_{i} \equiv 3(\bmod 4)$ para $i=1, \ldots, m$ e $m>0$, foi provado em [GA17] que $P\left(\mathbb{Z}_{n}\right)$ satisfaz a condição 5.2. Assim temos que $P\left(\mathbb{Z}_{n}\right)$ é um $A$-loop de ordem ímpar $n^{3}$. Além disso, para os mesmos valores de $n$, foi mostrado em [GA17] que o núcleo $N\left(P\left(\mathbb{Z}_{n}\right)\right)$ contém apenas o elemento identidade, implicando que $P\left(\mathbb{Z}_{n}\right)$ é não associativo.

Agora voltemos à análise de $(Q, *)$.

Proposição 5.1.8. $(Q, *)$ é não comutativo.

Demonstração. Como $(Q, *)$ é não associativo, então da proposição $5.1 .6 \mathrm{f})$, existem $x, y, z \in Q$, tais que $[x,[y, z]] \neq 0$. Assim, da proposição 5.1 .6 a) segue que $x$ e $[y, z]$ não comutam.

Para $x$ e $y$ pertencentes a $Q$, defina os comutadores à esquerda e à direita de $x$ e $y$ em $Q$, denotados respectivamente por $(x, y)_{L}$ e $(x, y)_{R}$, pelas seguintes expressões:

$$
x * y=(y * x) *(x, y)_{R} \quad x * y=(x, y)_{L} *(y * x)
$$

Defina os seguintes conjuntos:

$$
(Q, Q)_{R}=\left\{(x, y)_{R} \mid x, y \in Q\right\} \quad(Q, Q)_{L}=\left\{(x, y)_{L} \mid x, y \in Q\right\}
$$


Cabe observar aqui que como $(Q, *)$ é não comutativo, então os conjuntos $(Q, Q)_{L}$ e $(Q, Q)_{R}$ não são iguais à $\{0\}$.

Proposição 5.1.9. Seja $H=<(Q, Q)_{R}>$. Então:

a) $H$ é subloop característico de $Q$.

b) $H$ é subloop normal de $Q$.

c) $H$ contém $(Q, Q)_{L}$.

Demonstração. a) Fixado $\varphi \in \operatorname{Aut}(Q)$, sejam $x, y \in Q$. Assim $x * y=(y * x) *(x, y)_{R}$ e temos:

$$
\varphi(x) * \varphi(y)=(\varphi(y) * \varphi(x)) * \varphi\left((x, y)_{R}\right)
$$

$\operatorname{Logo} \varphi\left((x, y)_{R}\right)=(\varphi(x), \varphi(y))_{R}$. Desta forma, $\varphi\left((Q, Q)_{R}\right) \subseteq(Q, Q)_{R}$. Como $Q$ é finito e $\varphi$ é bijetora, temos que $(Q, Q)_{R}=\varphi\left((Q, Q)_{R}\right)$. Assim $(Q, Q)_{R} \subseteq \varphi(H)$. Como $\varphi(H)$ é subloop, segue que $H=<(Q, Q)_{R}>\subseteq \varphi(H)$. Novamente do fato de $Q$ ser finito e $\varphi$ ser bijetora, temos que $\varphi(H)=H$. Portanto $H$ é característico em $Q$.

b) Como $H$ é característico, da proposição 2.5.3 segue que $H$ é subloop normal de $Q$.

c) Fixados $x, y \in Q$, considere a classe lateral $H\left((x, y)_{L} *(y * x)\right)$. Pela definição em 5.12 temos que:

$$
H\left((x, y)_{L} *(y * x)\right)=H\left((y * x) *(x, y)_{R}\right)
$$

Como $H$ é subloop normal de $Q$, usando a propriedade de que $H z=z H$ na equação da direita temos:

$$
H\left((x, y)_{L} *(y * x)\right)=\left((y * x) *(x, y)_{R}\right) H
$$

Como $H$ contém $(Q, Q)_{R}$, segue que $\left((y * x) *(x, y)_{R}\right) H=(y * x) H$. Assim usando novamente a propriedade de que $\mathrm{Hz}=z H$ obtemos:

$$
H\left((x, y)_{L} *(y * x)\right)=H(y * x)
$$

Assim existe $h \in H$ tal que $(x, y)_{L} *(y * x)=h *(y * x)$. Logo $(x, y)_{L} \in H$.

Defina o seguinte conjunto:

$$
[Q, Q]=\{[x, y] \mid x, y \in Q\}
$$

Proposição 5.1.10. $N_{\mu}(Q)$ é subanel de Lie de $Q$. Além disso, $[Q, Q]$ está contido em $N_{\mu}(Q)$.

Demonstração. Relembramos que:

$$
N_{\mu}(Q)=\{a \in Q \mid[a,[z, w]]=0 \quad \forall z, w \in Q\}
$$

Da equação 5.4 segue que $[[x, y],[z, w]]=0$, para todo $x, y, z, w \in Q$. Assim, $[x, y] \in N_{\mu}(Q)$, para todo $x, y \in Q$. Logo $[Q, Q] \subseteq N_{\mu}(Q)$.

Agora fixados $x, y \in N_{\mu}(Q)$, para $z, w \in Q$, temos:

$$
[x-y,[z, w]]=[x,[z, w]]-[y,[z, w]]=0-0=0
$$

Logo $x-y \in N_{\mu}(Q)$, e temos que $N_{\mu}(Q)$ é subanel de Lie de $Q$. 
Seja $S$ um subconjunto não vazio de $Q$ e $x$ pertencente a $Q$. Definimos o centralizador de $S$ em $Q$, o centralizador de $x$ em $Q$ e o comutante de $Q$ respectivamente por:

$C_{Q}(S)=\{y \in Q \mid y * s=s * y, \quad \forall s \in S\}$

$C_{Q}(x)=\{y \in Q \mid x * y=y * x\}$

$C(Q)=\{y \in Q \mid y * z=z * y \quad \forall z \in Q\}$

Em $A$-loops, os três conjuntos acima são sempre subloops (proposição 2.5.6). Para estes conjuntos, temos os resultados seguintes.

Proposição 5.1.11. Com as mesmas notações anteriores, temos que:

a) $H$ é subloop de $N_{\mu}(Q)$

b) $N_{\mu}(Q)=C_{Q}(H)=C_{Q}\left((Q, Q)_{R}\right)$

Além disso, para todo $x$ e y pertencentes a $Q$ temos:

$$
(x, y)_{R}=-2[x, y]+\left[x+y,(x, y)_{R}\right]
$$

Demonstração. a) Fixados $x, y \in Q$, temos que $x * y=(y * x) *(x, y)_{R}$. Assim:

$$
\begin{aligned}
x+y-[x, y] & =x * y \\
& =(y * x) *(x, y)_{R} \\
& =(y+x-[y, x]) *(x, y)_{R} \\
& =x+y+[x, y]+(x, y)_{R}-\left[x+y,(x, y)_{R}\right]+\left[[y, x],(x, y)_{R}\right]
\end{aligned}
$$

Logo, $(x, y)_{R}=-2[x, y]+\left[x+y,(x, y)_{R}\right]-\left[[y, x],(x, y)_{R}\right]$.

Desta forma, da proposição 5.1.10, segue que $(x, y)_{R} \in N_{\mu}(Q)$. Como $H=<(Q, Q)_{R}>$, segue que $H$ é subloop de $N_{\mu}(Q)$ e assim fica provado a). Além disso, da expressão para $N_{\mu}(Q)$ dada em 5.7 segue que $\left[[y, x],(x, y)_{R}\right]=0$. Com isso temos:

$$
(x, y)_{R}=-2[x, y]+\left[x+y,(x, y)_{R}\right]
$$

b) Vamos mostrar que $C_{Q}(H)=C_{Q}\left((Q, Q)_{R}\right)=N_{\mu}(Q)$. Como $(Q, Q)_{R} \subseteq H$, já temos que $C_{Q}(H) \subseteq C_{Q}\left((Q, Q)_{R}\right)$. Logo, temos dois casos:

(i) Vamos mostrar que $C_{Q}\left((Q, Q)_{R}\right) \subseteq N_{\mu}(Q)$.

Fixado $a \in C_{Q}\left((Q, Q)_{R}\right)$, sejam $x, y \in Q$. Como $a *(x, y)_{R}=(x, y)_{R} * a$, da proposição 5.1.6 a) temos que $\left[a,(x, y)_{R}\right]=0$. Assim, usando a equação 5.13 temos:

$$
\left[a,-2[x, y]+\left[x+y,(x, y)_{R}\right]\right]=0
$$

Logo $-2[a,[x, y]]+\left[a,\left[x+y,(x, y)_{R}\right]\right]=0$. Com isso temos que:

$$
2[a,[x, y]]=\left[a,\left[x+y,(x, y)_{R}\right]\right]
$$

Usando a identidade de Jacobi temos:

$$
\left[a,\left[x+y,(x, y)_{R}\right]\right]+\left[x+y,\left[(x, y)_{R}, a\right]\right]+\left[(x, y)_{R},[a, x+y]\right]=0
$$

Usando a equação 5.14 segue que: 


$$
2[a,[x, y]]+\left[x+y,\left[(x, y)_{R}, a\right]\right]+\left[(x, y)_{R},[a, x+y]\right]=0
$$

Como $\left[a,(x, y)_{R}\right]=0$, temos que $\left[x+y,\left[(x, y)_{R}, a\right]\right]=0$. Além disso, do fato de $(x, y)_{R} \in N_{\mu}(Q)$ temos que $\left[(x, y)_{R},[a, x+y]\right]=0$. Logo $2[a,[x, y]]=0$. Como a ordem de $Q$ é ímpar, da expressão 5.8 segue que $[a,[x, y]]=0$.

Portanto $[a,[x, y]]=0$ para todo $x, y \in Q$, e assim da expressão para $N_{\mu}(Q)$ dada em 5.7 segue que $C_{Q}\left((Q, Q)_{R}\right) \subseteq N_{\mu}(Q)$.

(ii) Vamos mostrar que $N_{\mu}(Q) \subseteq C_{Q}(H)$.

Como $N_{\mu}(Q)$ é grupo finito e $H$ é subgrupo de $N_{\mu}(Q)$, basta mostrarmos que os elementos de $N_{\mu}(Q)$ comutam com os geradores de $H$. Fixado $a \in N_{\mu}(Q)$, para $(x, y)_{R} \in H$ usando a equação 5.13 temos:

$$
\begin{aligned}
{\left[a,(x, y)_{R}\right] } & =\left[a,-2[x, y]+\left[x+y,(x, y)_{R}\right]\right] \\
& =-2[a,[x, y]]+\left[a,\left[x+y,(x, y)_{R}\right]\right]
\end{aligned}
$$

Como $a \in N_{\mu}(Q)$, de 5.7 temos que $[a,[x, y]]$ e $\left[a,\left[x+y,(x, y)_{R}\right]\right]$ são nulos, logo $\left[a,(x, y)_{R}\right]=0$. Assim da proposição 5.1.6 a) segue que $a \in C_{Q}(H)$. Desta forma, $N_{\mu}(Q) \subseteq C_{Q}(H)$.

Portanto $C_{Q}(H)=C_{Q}\left((Q, Q)_{R}\right)=N_{\mu}(Q)$.

Proposição 5.1.12. Se x pertence a $H$, então $C_{Q}(x)$ é um subanel de Lie de $Q$ que contém $[Q, Q]$.

Demonstração. Fixado $x \in H$, como $N_{\mu}(Q)=C_{Q}(H)$, temos que $N_{\mu}(Q) \subseteq C_{Q}(x)$. Assim da proposição 5.1 .10 segue que $[Q, Q] \subseteq C_{Q}(x)$.

Agora note que da proposição 5.1.6 a) temos:

$$
C_{Q}(x)=\{y \in Q \mid[x, y]=0\}
$$

Assim, para $y, z \in C_{Q}(x)$ temos:

$$
[y-z, x]=[y, x]-[z, x]=0-0=0
$$

Logo $y-z \in C_{Q}(x)$, e temos que $C_{Q}(x)$ é subanel de Lie de $Q$.

\subsection{Half-Automorfismos de $(Q, *)$}

Como $Q$ é finito, do corolário 3.2.9 segue que todos os seus half-automorfismos são especiais. Assim, do teorema 3.2.7 e da proposição 3.2.10 a) segue o seguinte resultado:

Proposição 5.2.1. Seja $f: Q \rightarrow Q$ um half-automorfismo. Então

a) $\{f(x * y), f(y * x)\}=\{f(x) * f(y), f(y) * f(x)\}$, para todo $x$ e y pertencentes a $Q$.

b) Para todos $x$ e y pertencentes a $Q$ tais que $x * y=y * x$, temos $f(x) * f(y)=f(y) * f(x)$.

c) Se $M$ é subloop de $Q$, então $f(M)$ também é subloop de $Q$.

Dos itens a) e b) da proposição 5.2.1 decorre o resultado a seguir.

Corolário 5.2.2. Seja $f: Q \rightarrow Q$ um half-automorfismo. Então $x * y=y * x$ se, e somente se, $f(x) * f(y)=f(y) * f(x)$. 
Proposição 5.2.3. Sejam $f: Q \rightarrow Q$ um half-automorfismo e $H=<(Q, Q)_{R}>$. Então $f(H)=H$. Demonstração. Fixados $x, y \in Q$, vamos mostrar que $(f(x), f(y))_{R} \in f(H)$. Temos dois casos.

(i) Suponha que $f(x * y)=f(x) * f(y)$. Assim, da proposição 5.2.1, temos $f(y * x)=f(y) * f(x)$.

Como $x * y=(y * x) *(x, y)_{R}$, temos que $f(x * y)=f\left((y * x) *(x, y)_{R}\right)$. Logo:

$$
f(x) * f(y) \in\left\{(f(y) * f(x)) * f\left((x, y)_{R}\right), f\left((x, y)_{R}\right) *(f(y) * f(x))\right\}
$$

Como $f(x) * f(y)=(f(x), f(y))_{L} *(f(y) * f(x))$ e $f(x) * f(y)=(f(y) * f(x)) *(f(x), f(y))_{R}$, temos que $f\left((x, y)_{R}\right) \in\left\{(f(x), f(y))_{L},(f(x), f(y))_{R}\right\}$.

Agora, como $x * y=(x, y)_{L} *(y * x)$, temos que $f(x * y)=f\left((x, y)_{L} *(y * x)\right)$. Logo:

$$
f(x) * f(y) \in\left\{f\left((x, y)_{L}\right) *(f(y) * f(x)),(f(y) * f(x)) * f\left((x, y)_{L}\right)\right\}
$$

Desta forma, $f\left((x, y)_{L}\right) \in\left\{(f(x), f(y))_{L},(f(x), f(y))_{R}\right\}$. Logo:

$$
\left\{f\left((x, y)_{L}\right), f\left((x, y)_{R}\right)\right\} \subseteq\left\{(f(x), f(y))_{L},(f(x), f(y))_{R}\right\}
$$

(i.1) Vamos mostrar que $\left\{f\left((x, y)_{L}\right), f\left((x, y)_{R}\right)\right\}=\left\{(f(x), f(y))_{L},(f(x), f(y))_{R}\right\}$.

Se $f\left((x, y)_{L}\right) \neq f\left((x, y)_{R}\right)$, obtemos que o conjunto $\left\{f\left((x, y)_{L}\right), f\left((x, y)_{R}\right)\right\}$ possui dois elementos, o que implica que ele é igual ao conjunto $\left\{(f(x), f(y))_{L},(f(x), f(y))_{R}\right\}$.

Agora suponha que $f\left((x, y)_{L}\right)=f\left((x, y)_{R}\right)$. Como $f$ é bijetora, temos que $(x, y)_{L}=(x, y)_{R}$. Desta forma, $(x, y)_{L} *(y * x)=(x, y)_{R} *(y * x)$, e temos que:

$$
(y * x) *(x, y)_{R}=x * y=(x, y)_{L} *(y * x)=(x, y)_{R} *(y * x)
$$

Assim do item b) da proposição 5.2.1 temos que $f((y * x)) * f\left((x, y)_{R}\right)=f\left((x, y)_{R}\right) * f((y * x))$. Logo:

$$
(f(y) * f(x)) * f\left((x, y)_{R}\right)=f\left((x, y)_{R}\right) *(f(y) * f(x))
$$

Com isso, da equação 5.15 segue que:

$$
(f(y) * f(x)) * f\left((x, y)_{R}\right)=f\left((x, y)_{R}\right) *(f(y) * f(x))=f(x) * f(y)
$$

Desta forma, $(f(x), f(y))_{L}=(f(x), f(y))_{R}=f\left((x, y)_{R}\right)$, e temos que $\left\{f\left((x, y)_{L}\right), f\left((x, y)_{R}\right)\right\}=\left\{(f(x), f(y))_{L},(f(x), f(y))_{R}\right\}$.

Logo, $(f(x), f(y))_{R} \in\left\{f\left((x, y)_{L}\right), f\left((x, y)_{R}\right)\right\}$. Como $H$ contém $(x, y)_{R}$ e $(x, y)_{L}$ (proposição $5.1 .9 \mathrm{c}))$, temos que $f(H)$ contém $\left\{f\left((x, y)_{L}\right), f\left((x, y)_{R}\right)\right\}$. Assim, $(f(x), f(y))_{R} \in f(H)$.

(ii) Suponha que $f(x * y)=f(y) * f(x)$. Assim, da proposição 5.2.1, temos $f(y * x)=f(x) * f(y)$.

Como $y * x=(x * y) *(y, x)_{R}$, temos que $f(y * x)=f\left((x * y) *(y, x)_{R}\right)$. Logo:

$$
f(x) * f(y) \in\left\{(f(y) * f(x)) * f\left((y, x)_{R}\right), f\left((y, x)_{R}\right) *(f(y) * f(x))\right\}
$$

Assim, $f\left((y, x)_{R}\right) \in\left\{(f(x), f(y))_{L},(f(x), f(y))_{R}\right\}$.

Agora, como $y * x=(y, x)_{L} *(x * y)$, temos que $f(y * x)=f\left((y, x)_{L} *(x * y)\right)$. Logo: 


$$
f(x) * f(y) \in\left\{f\left((y, x)_{L}\right) *(f(y) * f(x)),(f(y) * f(x)) * f\left((y, x)_{L}\right)\right\}
$$

Desta forma, $f\left((y, x)_{L}\right) \in\left\{(f(x), f(y))_{L},(f(x), f(y))_{R}\right\}$. Logo:

$$
\left\{f\left((y, x)_{L}\right), f\left((y, x)_{R}\right)\right\} \subseteq\left\{(f(x), f(y))_{L},(f(x), f(y))_{R}\right\}
$$

(ii.1) Vamos mostrar que $\left\{f\left((y, x)_{L}\right), f\left((y, x)_{R}\right)\right\}=\left\{(f(x), f(y))_{L},(f(x), f(y))_{R}\right\}$.

Se $f\left((y, x)_{L}\right) \neq f\left((y, x)_{R}\right)$, obtemos que o conjunto $\left\{f\left((y, x)_{L}\right), f\left((y, x)_{R}\right)\right\}$ possui dois elementos, o que implica que ele é igual ao conjunto $\left\{(f(x), f(y))_{L},(f(x), f(y))_{R}\right\}$. Logo:

Agora suponha que $f\left((y, x)_{L}\right)=f\left((y, x)_{R}\right)$. Como $f$ é bijetora, segue que $(y, x)_{L}=(y, x)_{R}$.

$$
(x * y) *(y, x)_{R}=y * x=(y, x)_{L} *(x * y)=(y, x)_{R} *(x * y)
$$

Assim do item b) proposição 5.2.1 temos que $f((x * y)) * f\left((y, x)_{R}\right)=f\left((y, x)_{R}\right) * f((x * y))$. Logo:

$$
(f(y) * f(x)) * f\left((y, x)_{R}\right)=f\left((y, x)_{R}\right) *(f(y) * f(x))
$$

Com isso, da equação 5.16 segue que:

$$
(f(y) * f(x)) * f\left((y, x)_{R}\right)=f\left((y, x)_{R}\right) *(f(y) * f(x))=f(x) * f(y)
$$

Desta forma, $(f(x), f(y))_{L}=(f(x), f(y))_{R}=f\left((y, x)_{R}\right)$, e temos que $\left\{f\left((y, x)_{L}\right), f\left((y, x)_{R}\right)\right\}=\left\{(f(x), f(y))_{L},(f(x), f(y))_{R}\right\}$.

Logo, $(f(x), f(y))_{R} \in\left\{f\left((y, x)_{L}\right), f\left((y, x)_{R}\right)\right\} \subseteq f(H)$.

(iii) Portanto, temos que $(f(x), f(y))_{R} \in f(H)$, para todo $x, y \in Q$. Como $f$ é bijetora, segue que $(Q, Q)_{R} \subseteq f(H)$. Pelo item c) da proposição 5.2.1 temos que $f(H)$ é subloop de $Q$, logo $H=<(Q, Q)_{R}>$ está contido em $f(H)$. Assim, como $Q$ é finito e $f$ bijetora, segue que $f(H)=$ $H$.

Proposição 5.2.4. Seja $f: Q \rightarrow Q$ um half-automorfismo. Então $f\left(N_{\mu}(Q)\right)=N_{\mu}(Q)$.

Demonstração. Fixado $x \in N_{\mu}(Q)$, como $N_{\mu}(Q)=C_{Q}(H)$, então $x * h=h * x$ para todo $h$ pertencente a $H$. Assim, do item b) da proposição 5.2.1 temos:

$$
f(x) * f(h)=f(h) * f(x) \quad \forall h \in H
$$

Como da proposição 5.2.3 temos que $f(H)=H$, segue que:

$$
f(x) * h=h * f(x) \quad \forall h \in H
$$

Logo $f(x) \in C_{Q}(H)=N_{\mu}(Q)$. Portanto $f\left(N_{\mu}(Q)\right)$ está contido em $N_{\mu}(Q)$, e do fato de $f$ ser bijetora e $Q$ finito, temos a igualdade.

Em $A$-loops é válida a propriedade flexível, ou seja, $x *(y * x)=(x * y) * x$, para todo $x$ e $y$ pertencentes a $Q$. Para esta identidade, temos o resultado seguinte.

Proposição 5.2.5. Seja $f: Q \rightarrow Q$ um half-automorfismo. Então para todo $x$ e y pertencentes a $Q$ : 


$$
f(x * y * x)=f(x) * f(y) * f(x)
$$

Demonstração. Fixados $x, y \in Q$, temos dois casos:

(i) Suponha que $x * y=y * x$. Assim da proposição 5.2.1 segue que $f(x * y)=f(x) * f(y)=f(y) * f(x)$. Logo:

$$
f((x * y) * x) \in\{f(x * y) * f(x), f(x) * f(x * y)\}=\{f(x) * f(y) * f(x)\}
$$

(ii) Suponha que $x * y \neq y * x$. Assim, deste fato e do item b) da proposição 5.1.6 temos:

$$
x *(x * y) \neq(y * x) * x
$$

Do item b) da proposição 5.2.1, temos:

$$
\begin{aligned}
& \{f(x *(x * y)), f((x * y) * x)\}=\{f(x) * f(x * y), f(x * y) * f(x)\} \\
& \{f((y * x) * x), f(x *(y * x))\}=\{f(y * x) * f(x), f(x) * f(y * x)\}
\end{aligned}
$$

Com isso, temos mais dois casos:

(ii.1) Suponha que $f(x * y)=f(x) * f(y)$. Assim de 5.18 temos:

$\{f(x *(x * y)), f((x * y) * x)\}=\{f(x) *(f(x) * f(y)),(f(x) * f(y)) * f(x)\}$

$\{f((y * x) * x), f(x *(y * x))\}=\{(f(y) * f(x)) * f(x), f(x) *(f(y) * f(x))\}$

Logo, ou $f(x * y * x)=f(x) * f(y) * f(x)$ ou $f(x *(x * y))=f(x) * f(y) * f(x)=f((y * x) * x)$. Se $f(x *(x * y))=f(x) * f(y) * f(x)=f((y * x) * x)$, por $f$ ser bijetora segue que $x *(x * y)=(y * x) * x$, o que contraria a expressão 5.17. Portanto $f(x * y * x)=f(x) * f(y) * f(x)$.

(ii.2) Suponha que $f(x * y)=f(y) * f(x)$. Assim de 5.18 temos:

$\{f(x *(x * y)), f((x * y) * x)\}=\{f(x) *(f(y) * f(x)),(f(y) * f(x)) * f(x)\}$

$\{f((y * x) * x), f(x *(y * x))\}=\{(f(x) * f(y)) * f(x), f(x) *(f(x) * f(y))\}$

Desta forma, novamente obtemos que ou $f(x * y * x)=f(x) * f(y) * f(x)$ ou $f(x *(x * y))=f(x) * f(y) * f(x)=f((y * x) * x)$. Logo, analogamente ao caso (ii.1), da expressão 5.17 segue que $f(x * y * x)=f(x) * f(y) * f(x)$.

Definição 5.2.6. ([GGRS16]) Sejam $(L, *)$ e $\left(L^{\prime}, \cdot\right)$ loops e $\varphi: L \rightarrow L^{\prime}$ um half-isomorfismo. Para $x, y$ e $z$ pertencentes a $L$, a tripla $(x, y, z)$ é chamada tripla $G G$ de $\varphi$ se:

(i) $\varphi(x * y)=\varphi(x) \cdot \varphi(y) \neq \varphi(y) \cdot \varphi(x)$

(ii) $\varphi(x * z)=\varphi(z) \cdot \varphi(x) \neq \varphi(x) \cdot \varphi(z)$

Observação 5.2.7. No caso em que existe uma tripla GG de $\varphi$, o half-isomorfismo $\varphi$ é não trivial.

Observação 5.2.8. No caso em que $L=L^{\prime}=Q$ e existe $(x, y, z)$ tripla GG de $\varphi$, da proposição 5.2 .1 a) temos:

(i) $\varphi(y * x)=\varphi(y) \cdot \varphi(x)$ e $x * y$ é diferente de $y * x$.

(ii) $\varphi(z * x)=\varphi(x) \cdot \varphi(z)$ e $x * z$ é diferente de $z * x$. 
Agora, seja $f: Q \rightarrow Q$ um half-automorfismo. Defina os seguintes conjuntos:

$A=\{x \in Q \mid f(x * z)=f(x) * f(z) \quad \forall z \in Q\}$

$B=\{x \in Q \mid f(x * z)=f(z) * f(x) \quad \forall z \in Q\}$

Observe que 0 pertence tanto a $A$ quanto a $B$, assim esses conjuntos são não vazios. Observe também que $A=Q$ se, e somente se, $f$ é um automorfismo; e que $B=Q$ se, e somente se, $f$ é um anti-automorfismo. Assim $f$ é half-automorfismo trivial se, e somente se, $Q$ pertence a $\{A, B\}$.

Sejam $x$ pertencente a $A$ e $y$ pertencente a $B$. Assim $f(x * z)=f(x) * f(z)$ e $f(y * z)=f(z) * f(y)$, para todo $z$ pertencente a $Q$. Logo, da proposição 5.2 .1 a) temos:

$$
f(z * x)=f(z) * f(x) \quad \text { e } \quad f(z * y)=f(y) * f(z) \quad \forall z \in Q
$$

Com isto:

$$
f(x * y) \stackrel{x \in A}{=} f(x) * f(y) \stackrel{y \in B}{=} f(y * x)
$$

Logo, do fato de $f$ ser bijetora, segue que $x * y=y * x$. Assim, sendo $C_{Q}(x)=\{z \in Q \mid x * z=z * x\}$ o centralizador de $x$ em $Q$, temos:

$$
A \subseteq C_{Q}(y) \quad \text { e } \quad B \subseteq C_{Q}(x), \quad \forall x \in A, \quad \forall y \in B
$$

Proposição 5.2.9. $C(Q)=A \cap B$.

Demonstração. Fixado $x \in C(Q)$, para $y \in Q$ temos que $x * y=y * x$. Assim do item b) da proposição 5.2.1 temos que:

$$
f(x * y)=f(x) * f(y)=f(y) * f(x) \quad \forall y \in Q
$$

Logo, $x \in A \cap B$.

Agora fixado $x \in A \cap B$, seja $y \in Q$. Como $x \in A$, temos que $f(x * y)=f(x) * f(y)$. E como $x \in B$, temos que $f(x * y)=f(y) * f(x)$. Assim $f(x) * f(y)=f(y) * f(x)$ e do corolário 5.2.2 segue que $x * y=y * x$. Logo, $x \in C(Q)$.

A seguir serão enunciados e demonstrados seis lemas. Estes resultados serão fundamentais para a demonstração da proposição 5.2.16, enunciada após os mesmos.

Lema 5.2.10. Existem $x$ e y pertencentes a $Q$ tais que $(x, y)_{R}$ e $[x, y]$ não pertencem a $C(Q)$.

Demonstração. Pela demonstração da proposição 5.1.8 temos que existem $x, y, z \in Q$ tais que $[x, y]$ não comuta com $z$. Logo $[x, y]$ não pertence a $C(Q)$.

Agora suponha, por absurdo, que $(x, y)_{R} \in C(Q)$. Assim, $(x, y)_{R}$ comuta com $z$ e temos que $\left[(x, y)_{R}, z\right]=0$. Logo, usando a equação 5.13 para $(x, y)_{R}$ temos:

$$
\left[-2[x, y]+\left[x+y,(x, y)_{R}\right], z\right]=0
$$


Como $(x, y)_{R} \in C(Q)$, segue que $\left[x+y,(x, y)_{R}\right]=0$. Assim, $[-2[x, y], z]=0$, e temos que o seu oposto $2[[x, y], z]$ também é nulo. Como a ordem de $Q$ é ímpar, da expressão 5.8 segue que $[[x, y], z]=0$. Assim $[x, y]$ comuta com $z$, o que é um absurdo. Portanto $(x, y)_{R}$ não pertence a $C(Q)$.

Lema 5.2.11. Suponha que $N_{\mu}(Q)$ esteja contido em $A$ e que exista $x$ pertencente a $Q-A$. Então existe y pertencente a $H$ tal que:

$$
f(x * y)=f(x) * f(y) \neq f(y) * f(x) \text { e } x * y \neq y * x
$$

Demonstração. Como $x \notin A$, então $x \notin N_{\mu}(Q)$. Pela proposição 5.1.11 temos que $N_{\mu}(Q)=C_{Q}(H)$, então existe $y \in H$ tal que $x * y \neq y * x$. Assim do corolário 5.2.2 segue que $f(x) * f(y) \neq f(y) * f(x)$. Além disso, da proposição 5.1.11 também segue que $H$ é subloop de $N_{\mu}(Q)$, então $y \in A$. Logo, das equações em 5.19 temos que $f(x * y)=f(x) * f(y) \neq f(y) * f(x)$.

Para $x$ pertencente a $Q-A$ e $y$ pertencente a $H$ satisfazendo 5.21 , defina o conjunto $K_{x, y}$ como sendo:

$$
K_{x, y}=\{v \in Q \mid f(x * v)=f(v) * f(x) \neq f(x) * f(v)\}
$$

Como $x$ não pertence a $A$, então existe $w$ pertencente a $Q$ tal que

$$
f(x * w)=f(w) * f(x) \neq f(x) * f(w)
$$

Logo $w$ pertence a $K_{x, y}$, e assim este conjunto é não vazio.

Lema 5.2.12. Nas condições do lema anterior, sejam y pertencente a $H$ satisfazendo 5.21 e $z$ pertencente a $K_{x, y}$. Então $x+z$ e $x * z$ pertencem a $C_{Q}(y)$.

Demonstração. Como $z \in K_{x, y}$, então $f(x * z)=f(z) * f(x) \neq f(x) * f(z)$.

Seja $v \in Q$ tal que $z=y * v$. Como vimos no lema anterior, $H \subseteq N_{\mu}(Q) \subseteq A$, então $y \in A$. Assim temos que $f(z)=f(y) * f(v)$. Desta forma:

$$
f(z) * f(x)=f(x * z)=f(x *(y * v))
$$

Como $y \in N_{\mu}(Q)$, temos:

$$
f(z) * f(x)=f((x * y) * v) \in\{(f(x) * f(y)) * f(v), f(v) *(f(x) * f(y))\}
$$

Pela proposição 5.2.4 temos que $f(y) \in N_{\mu}(Q)$. Assim:

$$
(f(x) * f(y)) * f(v)=f(x) *(f(y) * f(v))=f(x) * f(z)
$$

Desta forma, $(f(x) * f(y)) * f(v) \neq f(z) * f(x)$. Logo, da equação 5.22 segue que $f((x * y) * v)=f(v) *(f(x) * f(y))$. Como $f(x) * f(y)=f(x * y)$, então $f((x * y) * v)=f(v) * f(x * y)$. Assim da proposição 5.2.1 a) e do fato de $f(y)$ pertencer a $N_{\mu}(Q)$ temos que:

$$
f(v *(x * y))=f(x * y) * f(v)=f(x) * f(y) * f(v)
$$

Como $f(z)=f(y) * f(v)$, então $f(v *(x * y))=f(x) * f(z)$. Como $f(x * z)=f(z) * f(x)$, da proposição 5.2.1 a) segue que $f(z * x)=f(x) * f(z)$, assim:

$$
f(v *(x * y))=f(z * x)=f((y * v) * x)
$$


Do fato de $f$ ser bijetora, segue que $v *(x * y)=(y * v) * x$. Assim, como $y \in N_{\mu}(Q)$, da proposição $5.1 .6 \mathrm{~d})$ temos que $[y, x+v]=0$. Portanto, $x+v \in C_{Q}(y)$. Com isso, temos que $y *(x+v)$ pertence a $C_{Q}(y)$. Além disso, para este elemento temos:

$$
\begin{aligned}
y *(x+v) & =y+x+v-[y, x+v] \\
& =y+x+v \\
& =y+v-[y, v]+x+[y, v] \\
& =y * v+x+[y, v] \\
& =z+x+[y, v]
\end{aligned}
$$

Logo $x+z=y *(x+v)-[y, v]$, e da proposição 5.1 .12 segue que $x+z \in C_{Q}(y)$. Assim, como $x * z=x+z-[x, z]$, da mesma proposição segue que $x * z \in C_{Q}(y)$.

Lema 5.2.13. Suponha que $N_{\mu}(Q)$ esteja contido em $B$ e que exista $x$ pertencente a $Q-B$. Então existe $z$ pertencente a $H$ tal que:

$$
f(x * z)=f(z) * f(x) \neq f(x) * f(z) \text { e } x * z \neq z * x
$$

Demonstração. Como $x \notin B$, então $x \notin N_{\mu}(Q)$. Pela proposição 5.1.11 temos que $N_{\mu}(Q)=C_{Q}(H)$, então existe $z \in H$ tal que $x * z \neq z * x$. Assim do corolário 5.2.2 segue que $f(x) * f(z) \neq f(z) * f(x)$. Além disso, da proposição 5.1.11 também segue que $H$ é subloop de $N_{\mu}(Q)$, então $z \in B$. Logo, das equações em 5.19 segue que $f(x * z)=f(z) * f(x) \neq f(x) * f(z)$.

Para $x$ pertencente a $Q-B$ e $z$ pertencente a $H$ satisfazendo 5.23 , defina o conjunto $K_{x, z}^{\prime}$ como sendo:

$$
K_{x, z}^{\prime}=\{v \in Q \mid f(x * v)=f(x) * f(v) \neq f(v) * f(x)\}
$$

Como $x$ não pertence a $B$, então existe $w$ pertencente a $Q$ tal que

$$
f(x * w)=f(x) * f(w) \neq f(w) * f(x)
$$

Logo $w$ pertence a $K_{x, z}^{\prime}$, e assim este conjunto é não vazio.

Lema 5.2.14. Nas condições do lema anterior, sejam z pertencente a $H$ satisfazendo 5.23 e y pertencente a $K_{x, z}^{\prime}$. Então $x+y$ e $x * y$ pertencem a $C_{Q}(z)$.

Demonstração. Como $y \in K_{x, z}^{\prime}$, então $f(x * y)=f(x) * f(y) \neq f(y) * f(x)$.

Seja $v \in Q$ tal que $y=z * v$. Como vimos no lema anterior, $H \subseteq N_{\mu}(Q) \subseteq B$, então $z \in B$. Assim temos que $f(y)=f(v) * f(z)$. Desta forma:

$$
f(x) * f(y)=f(x * y)=f(x *(z * v))
$$

Como $z \in N_{\mu}(Q)$, temos:

$$
f(x) * f(y)=f((x * z) * v) \in\{(f(z) * f(x)) * f(v), f(v) *(f(z) * f(x))\}
$$

Pela proposição 5.2.4 temos que $f(y) \in N_{\mu}(Q)$, assim:

$$
f(v) *(f(z) * f(x))=(f(v) * f(z)) * f(x)=f(y) * f(x)
$$


Desta forma, $f(v) *(f(z) * f(x)) \neq f(x) * f(y)$. Logo, da equação 5.24 segue que $f((x * z) * v)=(f(z) * f(x)) * f(v)$. Como $f(z) * f(x)=f(x * z)$, então $f((x * z) * v)=f(x * z) * f(v)$. Assim da proposição 5.2.1 a) e do fato de $f(y)$ pertencer a $N_{\mu}(Q)$ temos que:

$$
f(v *(x * z))=f(v) * f(x * z)=f(v) * f(z) * f(x)
$$

Como $f(y)=f(v) * f(z)$, então $f(v *(x * z))=f(y) * f(x)$. Como $f(x * y)=f(x) * f(y)$, da proposição 5.2.1 a) segue que $f(y * x)=f(y) * f(x)$, assim:

$$
f(v *(x * z))=f(y * x)=f((z * v) * x)
$$

Do fato de $f$ ser bijetora, segue que $v *(x * z)=(z * v) * x$. Assim, como $z \in N_{\mu}(Q)$, da proposição $5.1 .6 \mathrm{~d})$ temos que $[z, x+v]=0$. Portanto, $x+v \in C_{Q}(z)$. Com isso, temos que $z *(x+v)$ pertence a $C_{Q}(z)$. Além disso, para este elemento temos:

$$
\begin{aligned}
z *(x+v) & =z+x+v-[z, x+v] \\
& =z+x+v \\
& =z+v-[z, v]+x+[z, v] \\
& =z * v+x+[z, v] \\
& =y+x+[z, v]
\end{aligned}
$$

Logo $x+y=z *(x+v)-[z, v]$, e da proposição 5.1 .12 segue que $x+y \in C_{Q}(z)$. Assim, como $x * y=x+y-[x, y]$, da mesma proposição segue que $x * y \in C_{Q}(z)$.

Lema 5.2.15. Sejam a pertencente a $N_{\mu}(Q) \cap A$ e b pertencente a $N_{\mu}(Q) \cap B$. Então ou a pertence a $C(Q)$ ou b pertence a $C(Q)$.

Demonstração. Da equação 5.20 temos que $a * b=b * a$, assim para $w \in Q$ temos que $f(w *(b * a))=f(w *(a * b))$. Como $a \in N_{\mu}(Q)$ e $b \in B$, obtemos:

$$
f(w *(b * a))=f((w * a) * b)=f(b) * f(w * a)
$$

Usando o fato de que $a \in A$ e a expressão 5.19 , temos:

$$
f(w *(b * a))=f(b) *(f(w) * f(a))
$$

Por fim, usando o item b) da proposição 2.5.4, obtemos:

$$
f(w *(b * a))=f(b) * f(w) * f(a)
$$

Agora, da expressão 5.19 segue que $f(b * a)=f(b) * f(a)$. Assim, por $f$ ser um half-automorfismo, temos:

$$
f(w *(b * a)) \in\{f(w) *(f(b) * f(a)),(f(b) * f(a)) * f(w)\}
$$

Como $a, b \in N_{\mu}(Q)$, da proposição 5.2.4 segue que $f(a), f(b) \in N_{\mu}(Q)$. Então:

$$
f(w *(b * a)) \in\{f(w) * f(b) * f(a), f(b) * f(a) * f(w)\}
$$

Logo, da equação 5.25 temos:

$$
f(b) * f(w) * f(a) \in\{f(w) * f(b) * f(a), f(b) * f(a) * f(w)\}
$$


Vamos mostrar que $w \in C_{Q}(a) \cup C_{Q}(b)$. Temos dois casos:

i) Suponha que $f(b) * f(w) * f(a)=f(w) * f(b) * f(a)$.

Usando a lei do cancelamento, temos que $f(b) * f(w)=f(w) * f(b)$. Desta forma, do corolário 5.2 .2 segue que $b * w=w * b$. Logo, $w \in C_{Q}(b) \subseteq C_{Q}(a) \cup C_{Q}(b)$.

ii) Suponha que $f(b) * f(w) * f(a)=f(b) * f(a) * f(w)$.

Usando a lei do cancelamento, temos que $f(w) * f(a)=f(a) * f(w)$. Desta forma, do corolário 5.2.2 segue que $a * w=w * a$. Logo, $w \in C_{Q}(a) \subseteq C_{Q}(a) \cup C_{Q}(b)$.

Portanto, $w \in C_{Q}(a) \cup C_{Q}(b)$, para todo $w$ pertencente a $Q$. Logo, $Q=C_{Q}(a) \cup C_{Q}(b)$, e do lema 4.2.7 segue que $Q \in\left\{C_{Q}(a), C_{Q}(b)\right\}$.

É fácil ver que $Q=C_{Q}(t)$ se, e somente se, $t \in C(Q)$. Assim, temos que ou $a \in C(Q)$ ou $b \in C(Q)$.

Proposição 5.2.16. Seja $f: Q \rightarrow Q$ um half-automorfismo. Se $N_{\mu}(Q)$ está contido em $A \cup B$, então $f$ é half-automorfismo trivial.

Demonstração. Segue do lema 5.2.10 que existem $u, v \in Q$ tais que $(u, v)_{R} \notin C(Q)$. Como $H=<(Q, Q)_{R}>$, então $(u, v)_{R} \in H$. Além disso, como da proposição 5.1.11 temos que $H$ é subloop de $N_{\mu}(Q)$, então $(u, v)_{R}$ também pertence a $N_{\mu}(Q)$. Desta forma, como $N_{\mu}(Q) \subseteq A \cup B$, temos dois casos:

(i) Suponha que $a=(u, v)_{R} \in A$. Assim $a \in N_{\mu}(Q) \cap A$.

Fixado $b \in N_{\mu}(Q) \cap B$, do lema 5.2.15 segue que ou $a \in C(Q)$ ou $b \in C(Q)$. Como $a \notin C(Q)$, segue que $b \in C(Q)$.

Logo, $N_{\mu}(Q) \cap B \subseteq C(Q)$. Assim, da proposição 5.2.9 temos que $N_{\mu}(Q) \cap B \subseteq A$. Desta forma, como $N_{\mu}(Q) \subseteq A \cup B$, temos:

$$
N_{\mu}(Q)=\left(N_{\mu}(Q) \cap A\right) \cup\left(N_{\mu}(Q) \cap B\right) \subseteq A
$$

Agora suponha, por absurdo, que existe $x \in Q-A$. Pelo lema 5.2.11 temos que existe $y \in H$ tal que $x * y \neq y * x$ e assim o conjunto $K_{x, y}=\{w \in Q \mid f(x * w)=f(w) * f(x) \neq f(x) * f(w)\}$ é não vazio. Desta forma, existe $z \in K_{x, y}$. Logo, do lema 5.2.12 segue que $x * z \in C_{Q}(y)$.

Como $f(x *(x * z)) \in\{f(x) * f(x * z), f(x * z) * f(x)\}$, temos dois casos:

(i.1) Suponha que $f(x *(x * z))=f(x) * f(x * z)$.

Como $z \in K_{x, y}$, então $f(x * z)=f(z) * f(x)$, assim temos que $f(x *(x * z))=f(x) * f(z) * f(x)$. Com isso, da proposição 5.2.4 temos que $f(x *(x * z))=f(x * z * x)$. Como $f$ é bijetora, segue que $x *(x * z)=x * z * x$, e com isso obtemos que $x * z=z * x$. Logo, do item b) da proposição 5.2.1 temos que $f(x) * f(z)=f(z) * f(x)$, o que é um absurdo pois $z \in K_{x, y}$.

(i.2) Suponha que $f(x *(x * z))=f(x * z) * f(x) \neq f(x) * f(x * z)$. 
Assim, $x * z \in K_{x, y}$. Logo, do lema 5.2.12 segue que $x+(x * z) \in C_{Q}(y)$. Como $x * z \in C_{Q}(y)$, da proposição 5.1 .12 segue que $x \in C_{Q}(y)$. Assim $x * y=y * x$, o que é um absurdo.

Logo, não existe $x$ pertencente a $Q-A$ e assim temos que $f$ é um automorfismo.

(ii) Suponha que $b=(u, v)_{R} \in B$. Assim $b \in N_{\mu}(Q) \cap B$.

Fixado $a \in N_{\mu}(Q) \cap A$, do lema 5.2.15 segue que ou $a \in C(Q)$ ou $b \in C(Q)$. Como $b \notin C(Q)$, segue que $a \in C(Q)$.

Logo, $N_{\mu}(Q) \cap A \subseteq C(Q)$. Assim, da proposição 5.2.9 temos que $N_{\mu}(Q) \cap A \subseteq B$. Desta forma, como $N_{\mu}(Q) \subseteq A \cup B$, temos:

$$
N_{\mu}(Q)=\left(N_{\mu}(Q) \cap A\right) \cup\left(N_{\mu}(Q) \cap B\right) \subseteq B
$$

Agora suponha, por absurdo, que existe $x \in Q-B$. Pelo lema 5.2.13 temos que existe $z \in H$ tal que $x * z \neq z * x$ e assim o conjunto $K_{x, z}^{\prime}=\{w \in Q \mid f(x * w)=f(x) * f(w) \neq f(w) * f(x)\}$ é não vazio. Desta forma, existe $y \in K_{x, z}^{\prime}$. Logo, do lema 5.2.14 segue que $x * y \in C_{Q}(z)$.

Como $f(x *(x * y)) \in\{f(x) * f(x * y), f(x * y) * f(x)\}$, temos dois casos:

(ii.1) Suponha que $f(x *(x * y))=f(x * y) * f(x)$.

Como $y \in K_{x, z}^{\prime}$, então $f(x * y)=f(x) * f(y)$, assim temos que $f(x *(x * y))=f(x) * f(y) * f(x)$. Com isso, da proposição 5.2.4 temos que $f(x *(x * y))=f(x * y * x)$. Como $f$ é bijetora, segue que $x *(x * y)=x * y * x$, e com isso obtemos que $x * y=y * x$. Logo, do item b) da proposição 5.2.1 temos que $f(x) * f(y)=f(y) * f(x)$, o que é um absurdo pois $y \in K_{x, z}^{\prime}$.

(ii.2) Suponha que $f(x *(x * y))=f(x) * f(x * y) \neq f(x * y) * f(x)$.

Assim, $x * y \in K_{x, z}^{\prime}$. Logo, do lema 5.2.14 segue que $x+(x * y) \in C_{Q}(z)$. Como $x * y \in C_{Q}(z)$, da proposição 5.1 .12 segue que $x \in C_{Q}(z)$. Assim $x * z=z * x$, o que é um absurdo.

Logo, não existe $x$ pertencente a $Q-B$ e assim temos que $f$ é um anti-automorfismo.

Lema 5.2.17. Seja $f: Q \rightarrow Q$ um half-automorfismo e suponha que exista $x$ pertencente a $Q$ tal que $x$ não pertence a $A \cup B$. Então existem y e $z$ pertencentes a $Q$ tais que $y * z$ é diferente de $z * y$ e que $(x, y, z)$ é tripla $G G$ de $f$.

Demonstração. Como $x \notin A$, então existe $z_{0} \in Q$ tal que $f\left(x * z_{0}\right)=f\left(z_{0}\right) * f(x) \neq f(x) * f\left(z_{0}\right)$, e do corolário 5.2.2 segue que $x * z_{0} \neq z_{0} * x$.

Como $x \notin B$, então existe $y \in Q$ tal que $f(x * y)=f(x) * f(y) \neq f(y) * f(x)$, e com isso $x * y \neq y * x$. Desta forma, $C_{Q}(y) \neq Q$.

Suponha, por absurdo, que:

$$
f(x * v)=f(x) * f(v) \quad \forall v \in Q-C_{Q}(y)
$$

Como $f\left(x * z_{0}\right) \neq f(x) * f\left(z_{0}\right)$, então $z_{0} \in C_{Q}(y)$. Como $x$ não comuta com $y$, então $x \notin C_{Q}(y)$. Assim, como $C_{Q}(y)$ é subloop de $Q$, segue que $x * z_{0} \in Q-C_{Q}(y)$. Logo, da equação 5.26 temos: 


$$
f\left(x *\left(x * z_{0}\right)\right)=f(x) * f\left(x * z_{0}\right)
$$

Como $f\left(x * z_{0}\right)=f\left(z_{0}\right) * f(x)$, temos que $f\left(x *\left(x * z_{0}\right)\right)=f(x) * f\left(z_{0}\right) * f(x)$. Logo, da proposição 5.2.5 segue que $f\left(x *\left(x * z_{0}\right)\right)=f\left(x * z_{0} * x\right)$. Do fato de $f$ ser bijetora, segue que $x *\left(x * z_{0}\right)=x * z_{0} * x$, e com isso temos que $x * z_{0}=z_{0} * x$, o que é um absurdo.

Portanto, existe $z \in Q-C_{Q}(y)$ tal que $f(x * z) \neq f(x) * f(z)$. Logo, $(x, y, z)$ é tripla GG de $f$ e $y * z \neq z * y$.

Teorema 5.2.18. Todo half-automorfismo de $Q$ é trivial.

Demonstração. Seja $f: Q \rightarrow Q$ um half-automorfismo. Note que pela proposição 5.2 .16 basta mostrarmos que $N_{\mu}(Q) \subseteq A \cup B$.

Suponha, por absurdo, que existe $x \in N_{\mu}(Q)$ tal que $x \notin A \cup B$. Assim, do lema 5.2.17 segue que existem $y, z \in Q$ tais que $y * z \neq z * y$ e $(x, y, z)$ é tripla GG de $f$.

(i) Vamos mostrar que $f(z) * f(x) * f(y)=f(y) * f(x) * f(z)$.

Usando a definição de half-automorfismo e que $(x, y, z)$ é tripla $\mathrm{GG}$, temos:

$f(y *(x * z)) \in\{f(y) *(f(z) * f(x)),(f(z) * f(x)) * f(y)\}$

$f((y * x) * z) \in\{(f(y) * f(x)) * f(z), f(z) *(f(y) * f(x))\}$

Como $x \in N_{\mu}(Q)$, da proposição 5.2.4 temos que $f(x) \in N_{\mu}(Q)$. Além disso, do fato de $x$ pertencer a $N_{\mu}(Q)$ também segue que $f(y *(x * z))=f((y * x) * z)$. Logo, temos quatro casos:

(i.1) Suponha que $f(y * x * z)=f(y) *(f(z) * f(x))=(f(y) * f(x)) * f(z)$.

Como $f(x) \in N_{\mu}(Q)$, então $f(y) *(f(z) * f(x))=f(y) * f(x) * f(z)$, e usando a lei do cancelamento obtemos que $f(z) * f(x)=f(x) * f(z)$, o que é um absurdo, pois $(x, y, z)$ é tripla GG.

(i.2) Suponha que $f(y * x * z)=f(y) *(f(z) * f(x))=f(z) *(f(y) * f(x))$.

Como $f(x) \in N_{\mu}(Q)$, da proposição 5.1.6 c) temos que $f(y) * f(z)=f(z) * f(y)$, logo do corolário 5.2.2 segue que $y * z=z * y$, o que é um absurdo.

(i.3) Suponha que $f(y * x * z)=(f(z) * f(x)) * f(y)=f(z) *(f(y) * f(x))$.

Como $f(x) \in N_{\mu}(Q)$, então $f(z) * f(x) * f(y)=f(z) *(f(y) * f(x))$, e usando a lei do cancelamento obtemos que $f(x) * f(y)=f(y) * f(x)$, o que é um absurdo, pois $(x, y, z)$ é tripla GG.

Portanto, do único caso restante e do fato de que $f(x) \in N_{\mu}(Q)$ segue que $f(y * x * z)=$ $f(z) * f(x) * f(y)=f(y) * f(x) * f(z)$. Assim, da proposição 5.1.6 e) temos:

$$
\begin{aligned}
& (f(x) * f(y)) *(f(x) * f(z))=(f(x) * f(z)) *(f(x) * f(y)) \\
& (f(y) * f(x)) *(f(z) * f(x))=(f(z) * f(x)) *(f(y) * f(x))
\end{aligned}
$$

(ii) Usando a proposição 5.2.5, a definição de half-automorfismo e que $(x, y, z)$ é tripla GG, temos: 
$f((x * y * x) * z) \in\{(f(x) * f(y) * f(x)) * f(z), f(z) *(f(x) * f(y) * f(x))\}$

$f((x * y) *(x * z)) \in\{(f(x) * f(y)) *(f(z) * f(x)),(f(z) * f(x)) *(f(x) * f(y))\}$

Como $x \in N_{\mu}(Q)$, então $(x * y * x) * z=(x * y) *(x * z)$. Logo, $f((x * y * x) * z)=f((x * y) *(x * z))$, e assim temos quatro casos:

(ii.1) Suponha que $f((x * y * x) * z)=(f(x) * f(y) * f(x)) * f(z)=(f(x) * f(y)) *(f(z) * f(x))$.

Como $f(x) \in N_{\mu}(Q)$, então:

$$
(f(x) * f(y)) *(f(x) * f(z))=(f(x) * f(y)) *(f(z) * f(x))
$$

Logo $f(x) * f(z)=f(z) * f(x)$ e temos um absurdo, pois $(x, y, z)$ é tripla GG.

(ii.2) Suponha que $f((x * y * x) * z)=(f(x) * f(y) * f(x)) * f(z)=(f(z) * f(x)) *(f(x) * f(y))$.

Como $f(x) \in N_{\mu}(Q)$, então $(f(x) * f(y)) *(f(x) * f(z))=(f(z) * f(x)) *(f(x) * f(y))$. Assim, usando a equação 5.27 temos:

$$
(f(x) * f(z)) *(f(x) * f(y))=(f(x) * f(y)) *(f(x) * f(z))=(f(z) * f(x)) *(f(x) * f(y))
$$

Logo, $f(x) * f(z)=f(z) * f(x)$ e temos um absurdo.

(ii.3) Suponha que $f((x * y * x) * z)=f(z) *(f(x) * f(y) * f(x))=(f(x) * f(y)) *(f(z) * f(x))$.

Como $f(x) \in N_{\mu}(Q)$, então $(f(z) * f(x)) *(f(y) * f(x))=(f(x) * f(y)) *(f(z) * f(x))$. Assim, usando a equação 5.28 temos:

$$
(f(y) * f(x)) *(f(z) * f(x))=(f(z) * f(x)) *(f(y) * f(x))=(f(x) * f(y)) *(f(z) * f(x))
$$

Logo, $f(x) * f(y)=f(y) * f(x)$ e temos um absurdo, pois $(x, y, z)$ é tripla GG.

(ii.4) Suponha que $f((x * y * x) * z)=f(z) *(f(x) * f(y) * f(x))=(f(z) * f(x)) *(f(x) * f(y))$.

Como $f(x) \in N_{\mu}(Q)$, então:

$$
(f(z) * f(x)) *(f(y) * f(x))=(f(z) * f(x)) *(f(x) * f(y))
$$

Logo, $f(x) * f(y)=f(y) * f(x)$ e temos um absurdo.

Portanto, $N_{\mu}(Q) \subseteq A \cup B$ e da proposição 5.2 .16 segue o resultado. 


\section{Referências Bibliográficas}

[AV16] M. Aboras e P. Vojtěchovský. Automorphisms of Dihedral-Like Automorphic Loops. Communications in Algebra, 44:613-627, 2016. 23, 25, 36

[BP56] R.H. Bruck e L.J. Paige. Loops whose inner mappings are automorphisms. Annals of Mathematics, 63:308-323, 1956. 1, 12, 13

[dA14] Giliard Souza dos Anjos. Grupo de automorfismos de a-loops. Dissertação de Mestrado, Universidade Federal do ABC, Brasil, Fevereiro 2014. 23, 24

[Drá10] A. Drápal. A Simplified Proof of Moufang's Theorem. Proceedings of the American Mathematical Society, 139:93-98, 2010. 12

[GA17] M.L. Merlini Giuliani e G.S. Anjos. On the structure of the automorphism group of certain automorphic loop. Communications in Algebra, 45(5):2245-2255, 2017. 43, 48

[GGRS16] A. Grishkov, M.L. Merlini Giuliani, M. Rasskazova e L. Sabinina. Half-isomorphisms of finite Automorphic Moufang loops. Communications in Algebra, 44:4252-4261, 2016. 1,54

[GJM96] E.G. Goodaire, E. Jespers e C. Polcino Milies. Alternative Loop Rings. Amsterdam, 1996. 5,12

[Gro] The GAP Group. GAP - group, algorithms and programming, version 4.8.7, 2017. 12, 15

[IG12] S. Gagola III e M.L. Merlini Giuliani. Half-isomorphisms of Moufang loops of odd order. Journal of Algebra and Its Applications, 11:194-199, 2012. 1

[IG13] S. Gagola III e M.L. Merlini Giuliani. On half-automorphisms of certain Moufang loops with even order. Journal of Algebra, 386:131-141, 2013. 1

[KKP02] M.K. Kinyon, K. Kunen e J.D. Phillips. Every diassociative A-loop is Moufang. Proceedings of the American Mathematical Society, 130:619-624, 2002. 1

[KKPV16] M.K. Kinyon, K. Kunen, J.D. Phillips e P. Vojtěchovský. The Structure of Automorphic Loops. Transactions of the American Mathematical Society, 368:8901-8927, 2016. 1, $13,23,24,43,44$

[KSV16] M.K. Kinyon, I. Stuhl e P. Vojtěchovský. Half-Isomorphisms of Moufang Loops. Journal of Algebra, 450:152-161, 2016. 1

[Mou35] R. Moufang. Zur Struktur von Alternativ Korpern. Mathematische Annalen, 110:416430, 1935. 1

[Pfl90] H.O. Pflugfelder. Quasigroups and Loops: Introduction, volume 7 of Sigma Series in Pure Mathematics. Heldermann Verlag Berlin, 1990. 4, 12

[Sco57] W.R. Scott. Half-homomorphisms of groups. Proceedings of the American Mathematical Society, 8:1141-1144, 1957. 1 
[Wri69] C.R.B. Wright. On the multiplication group of a loop. Illinois Journal of Mathematics, 13:660-673, 1969. 1, 43 